\title{
RECENT DEVELOPMENTS ON PSEUDO-DIFFERENTIAL OPERATORS (II)
}

\author{
DER-CHEN CHANG, XIAOJING LYU AND BERT-WOLFGANG SCHULZE
}

\begin{abstract}
The analysis on manifolds with singularities is a rapidly developing field of research, with new achievements and compelling challenges. We present here elements of an iterative approach to building up pseudo-differential structures. Those participate in operator algebras on singular manifolds and reflect the properties of parametrices of elliptic operators, including boundary value problems.
\end{abstract}

\section{Introduction}

The relationship between partial differential operators and their symbols is the origin of the calculus of pseudo-differential operators, where the symbols are not necessarily polynomials in the covariables. Basics have been well-known through the works of Kohn and Nirenberg [29], Hörmander [26], and many other authors. Standard material can be found in numerous textbooks on this topic. From the very beginning of its development the pseudodifferential analysis interacted with other fields of mathematics and applications in natural sciences, especially, geometry, topology, and physics. New aspects and recent achievements are outlined in [13]. In the present Part II we give more insight into pseudo-differential techniques from the analysis on manifolds with singularities, with new starting points and future possibilities and challenges.

The classical ideas around ellipticity of operators, parametrices, and index on smooth closed manifolds are an inspiration also for the singular analysis. The same is true of other traditional structures occurring in machineries for solving parabolic or hyperbolic problems. The singular geometries in such contexts cause new and partly unexpected problems. Although those are sometimes really hard, the new symbolic structures and iterative ideas that are created so far are very beautiful. Also the remarkable progress during the past decades is

Received November 19, 2014, accepted March 19, 2015.

2010 Mathematics Subject Classification. Primary 35S35; Secondary 35J70.

Key words and phrases. Pseudo-differential operators, boundary value problems, operator-valued symbols, Fourier transform.

Corresponding author: D.-C. Chang. 
an encouragement to participate in these fields. In this exposition we focus on what is necessary for elliptic operators. Later on in other parts of this series we also turn to anisotropic symbolic structures and parabolicity.

The interactions between classical pseudo-differential analysis and other fields of mathematics have attracted mathematicians since a long time. One of the most impressive examples is the index theory of elliptic operators and of boundary value problems (BVPs) on manifolds. In the article [21] Gelfand pointed out that the Fredholm index of a Shapiro-Lopatinskij elliptic BVP is a function of the involved principal symbols alone. This lead to the question, known as Gelfand's program, how to express the index purely in terms of suitable equivalence classes of symbols. In the subsequent development more aspects and objectives have been included in the discussion, in particular, operator algebras, complex analysis, algebraic topology, homotopy theory, and $K$-theory. The development culminated in the Atiyah-Singer index theorem, cf. [3]. These achievements first concerned elliptic operators on a smooth closed manifold, where the ellipticity of a classical (pseudo-) differential operator is determined by its homogeneous principal symbol (and topological data of the underlying manifold). Later on the development integrated boundary value problems for operators with the transmission property at the boundary, cf. Boutet de Monvel [7]. It has been assumed in this paper that a topological obstruction for the interior symbol vanishes, cf. Atiyah and Bott [4] for the case of differential operators. During this period also operators without the transmission property have been investigated, see the theory of pseudo-differential BVPs of Vishik and Eskin [59], [60], and Eskin [18], based on a higher-dimensional analogue of Wiener-Hopf techniques. Also questions about parametrices and the index have been considered in these works.

A "well-organized" pseudo-differential theory should be able to express the parametrices of elliptic operators within a corresponding operator algebra. This is the case in "standard" pseudo-differential operators on a closed smooth manifold. Then the ellipticity of the corresponding operator is equivalent to its Fredholm property in standard Sobolev spaces. If the manifold is non-compact and smooth, ellipticity and parametrices can be formulated as well. However, the nature of "coefficients" of operators and also the kind of smoothing operators close to the non-compact ends of the manifold are very essential for Fredholmness and index in (which kind of?) Sobolev spaces. The difference between different notions of ellipticity and index theory can be illustrated by comparing different compactifications of the respective manifold. For instance, if $M$ is a smooth compact manifold, $v \in M$ a single point, then $M \backslash\{v\}$ can be first compactified to $M_{0}:=M$ itself. However, when $v$ is regarded as an embedded conical singularity we write $M_{1}$ rather than $M$. Another compactification $M_{2}$ of $M \backslash\{v\}$ consists of a manifold with smooth boundary, where $\partial M_{2}$ is a sphere of dimension $\operatorname{dim} M-1$. While for $M_{0}$ we have the usual pseudo-differential calculus on $M$ in the smooth sense across $v$, on $M_{1}$ there is the calculus on $M_{1} \backslash\{\nu\}$ with $M_{1}$ as a manifold with conical singularity $v$, which 
is rather different from the smooth one close to $v$. Moreover, $\partial M_{2}$ admits the completely different calculus of BVPs. We then have even different choices of non-equivalent theories, e.g., BVPs with or without the transmission property at the boundary. The spaces $M_{0}, M_{1}$ and $M_{2}$ are examples of stratified spaces, and the indicated pseudo-differential structures are determined by corresponding symbolic hierarchies, the components of which are contributed by the involved strata. We can also compactify $M \backslash\{v\}$ in such a way that the boundary is a manifold with edges or corners, i.e., stratified again. Then for operators and their ellipticity we need symbolic hierarchies of more than 2 components.

The idea of this paper is to pick some crucial parts of recent inventions in singular analysis which are necessary for the indicated pseudo-differential structures and to establish new insight and contributions to building up singular operators in corresponding algebras with symbolic structures.

\section{Manifolds with singularities}

\subsection{Hierarchies of stratified spaces}

Manifolds appear in numerous models of physics. For instance, the boundary of a smooth domain in Euclidean space $\mathbb{R}^{n}$ is a manifold of dimension $n-1$. It is then of interest to study harmonic functions in the domain with respect to their behaviour at the boundary. Denoting the domain including its boundary by $M$, we have an example of a smooth manifold with boundary, while the open interior is a smooth manifold in the "usual" sense, i.e., every point has a neighbourhood that is differomorphic to $\mathbb{R}^{n}$. Dirichlet or Neumann problems for the Laplacian are classical examples of elliptic BVPs. Those can be represented by a row matrix operator

$$
\mathscr{A}=\left(\begin{array}{c}
A \\
T_{j}
\end{array}\right),
$$

$j=0,1$, where $A$ stands for the Laplace operator $\Delta=\sum_{i=1}^{n} \frac{\partial^{2}}{\partial x_{i}^{2}}$, and $T_{0}$ for the restriction of a function to the boundary, $T_{1}$ for the restriction of the normal derivative to the boundary. As is well-known, smoothness of right hand sides of $\mathscr{A} u={ }^{\mathrm{t}}(f, g)$, i.e., $f \in C^{\infty}(M), g \in C^{\infty}(\partial M)$, entails smoothness of solutions $u$ up to the boundary. This is a special case of elliptic regularity. More generally, $f \in H^{s-2}$ (int $M$ ), $g \in H^{s-1 / 2}(\partial M$ ) (for compact $M$ and $s>3 / 2$ ) gives rise to $u \in H^{s}$ (int $M$ ). However, if $M$ is not smooth, for instance, a cone or a cube, then it is far from being obvious, in which kind of spaces we can formulate elliptic regularity, and, in particular, how to substitute the smoothness of solutions up to the singular points of the boundary. Also unique solvability or Fredholmness of the operator can break down. What concerns the symbolic structure of parametrices close to the boundary, even in the case of a smooth boundary, the answer in terms of interior and boundary symbols is not obvious, although known by 
Boutet de Monvel's calculus, cf. [7] or Rempel and Schulze [35]. Non-smooth, e.g., piecewise smooth boundaries, require the tools of the singular analysis, for instance, of operators on stratified spaces. Other important problems are mixed, transmission and crack problems, cf. [27], [24], which require the analysis on manifolds with conical and edge singularities.

A prototype of a mixed elliptic problem is the Zaremba problem for the Laplacian. In this case the boundary is subdivided into submanifolds $(\partial M)_{ \pm}$, i.e., $\partial M=(\partial M)_{-} \cup(\partial M)_{+}$, with common boundary $(\partial M)_{-} \cap(\partial M)_{+}:=Z$ of codimension 1 which is in simplest cases smooth. In the Zaremba problem there are posed Dirichlet conditions on $(\partial M)_{-}$, Neumann conditions on $(\partial M)_{+}$. This problem for a smooth interface has been investigated also in [10] from the point of view of the edge calculus of BVPs, where the interface is the edge. It is also important to admit interfaces with singularities, e.g., with conical points, edges, or corners. Then the corresponding configuration is a stratified space of a higher depth. These examples show that singular manifolds play the role of underlying spaces for interesting analytic objects, here BVPs for (pseudo-)differential operators. More examples come from particle physics, quantum chemistry, material sciences, and other fields of applications.

For a topological space $B$ we set

$$
B^{\triangle}:=\left(\overline{\mathbb{R}}_{+} \times B\right) /(\{0\} \times B) \text { and } B^{\wedge}:=\mathbb{R}_{+} \times B .
$$

The space $B$ is often called the link of the cone $B^{\wedge}$ or of the open stretched cone $B^{\wedge}$. In our considerations below we fix a splitting of variables

$$
(r, \cdot) \in B^{\wedge}
$$

and only admit other splittings, regarded to be regular with respect to the fixed one, when the transition $(r, \cdot) \rightarrow(\tilde{r}, \tilde{)})$ is smooth up to $r=0$ in a sense to be defined below after Definition 1 . In an iterative manner we construct categories $\mathfrak{M}_{k}$ of stratified spaces of increasing singularity orders, with $\mathfrak{M}_{0}$, being the system of smooth manifolds. We then assume that $\mathfrak{M}_{k-1}$ for $k \in$ $\mathbb{N}, k \geq 1$, is already introduced, and we pass to $\mathfrak{M}_{k}$.

\section{Definition 1 ([48]).}

(i) A (paracompact) topological space $M$ belongs to $\mathfrak{M}_{k}$ for $k \in \mathbb{N}, k \geq 1$, if there is a subset $s_{k}(M) \subseteq M, s_{k}(M) \in \mathfrak{M}_{0}$, such that $M \backslash s_{k}(M) \in \mathfrak{M}_{k-1}$.

(ii) There is a neighbourhood $V$ of $s_{k}(M)$ in $M$ with the structure of a locally trivial regular $B^{\triangle}$-bundle over $s_{k}(M)$ for some $B \in \mathfrak{M}_{k-1}$.

Definition 1 (ii) refers to bundles over a smooth manifold $X$, where the fibre is a cone. The meaning is similar to a vector bundle $E$ over $X$ with bundle projection $\pi: E \rightarrow X$ and fibre $F$ which is, say, a finite-dimensional complex vector space. A possible definition is based on 
a system of trivialisations $\pi^{-1} U:=\left.E\right|_{U} \cong U \times F$, where $U$ runs over an open covering of $X$ and on a given cocycle of transition maps $\chi_{V, U}^{E}:(U \cap V) \times F \rightarrow(U \cap V) \times F$ with $U, V$ belonging to the open covering. For $B^{\triangle}$ instead of $F$ we denote the corresponding bundle by $E^{\triangle}$. In order to define regular singularities (not cuspidal ones) we ask the existence of bundles $E^{\wedge}$ with fibre $B^{\wedge}, E^{0}$ with fibre $B$, and $\mathbb{E}$ with fibre $\mathbb{R} \times B$, such that both $E^{\wedge}$ and $E^{0}$ are subbundles of $\mathbb{E}$ determined by the inclusions of fibres $B^{\wedge} \subset \mathbb{R} \times B$ and $B \subset \mathbb{R} \times B$, the latter realised as $\{0\} \times B$, and that then the transition maps for $E^{\triangle}$

$$
\chi_{V, U}^{E^{\Delta}}:(U \bigcap V) \times B^{\Delta} \rightarrow(U \bigcap V) \times B^{\Delta}
$$

are obtained as quotient maps $\chi_{V, U}^{E^{\Delta}}=\chi_{V, U}^{\overline{\mathbb{E}}_{+}} / \chi_{V, U}^{E^{0}}$, where $\chi_{V, U}^{\overline{\mathbb{E}}_{+}}$is the cocycle of transition maps for $\overline{\mathbb{E}}_{+}$, the subbundle of $\mathbb{E}$ with fibre $\overline{\mathbb{R}}_{+} \times B$, and $\chi_{V, U}^{E^{0}}$ is the induced cocycle for $E^{0}$ as a subbundle of $\overline{\mathbb{E}}_{+}$.

In addition we require homogeneity of

$$
\chi_{V, U}^{\overline{\mathbb{E}}_{+}}:(U \bigcap V) \times\left(\overline{\mathbb{R}}_{+} \times B\right) \rightarrow(U \bigcap V) \times\left(\overline{\mathbb{R}}_{+} \times B\right)
$$

in the sense

$$
\chi_{V, U}^{\overline{\mathbb{E}}_{+}}(x, \delta r, \cdot)=\chi_{V, U}^{\overline{\mathbb{E}}_{+}}(x, r, \cdot)
$$

for every $\delta \in \mathbb{R}_{+}$and $(x, r, \cdot) \in(U \cap V) \times\left(\overline{\mathbb{R}}_{+} \times B\right)$. In other words, fibres $\overline{\mathbb{R}}_{+} \times B$ or $B^{\triangle}$ are transformed via transforming the Cartesian product $\overline{\mathbb{R}}_{+} \times B$ by id $\overline{\mathbb{R}}_{+} \times \beta$, where $\beta: B \rightarrow B$ is an isomorphism in $\mathfrak{M}_{k-1}$. Isomorphisms $\beta$ can be defined in an iterative manner, beginning from $B \in \mathfrak{M}_{0}$ where isomorphisms are simply diffeomorphisms. The iterative definition of isomorphisms in $\mathfrak{M}_{k}$ for $k \in \mathbb{N}, k \geq 1$, is straightforward.

Remark 1. A topological space $M$ belongs to $\mathfrak{M}_{k}$ for $k \in \mathbb{N}, k \geq 1$, if

(i) $M$ is stratified, i.e., there is singled out a sequence

$$
s(M):=\left(s_{0}(M), s_{1}(M), \ldots, s_{k}(M)\right)
$$

of subsets $s_{j}(M) \subseteq M$ belonging to $\mathfrak{M}_{0}, j=0, \ldots, k$, such that

$$
s_{j}(M) \bigcap s_{l}(M)=\varnothing \quad \text { for all } j \neq l, \quad M=\bigcup_{j=0}^{k} s_{j}(M),
$$

and

$$
\operatorname{dim} s_{j}(M)>\operatorname{dim} s_{l}(M) \text { for } j<l \text { for } 0 \leq j \leq k-1 \text {. }
$$

(ii) We have

$$
M_{j}:=M \backslash \bigcup_{i=j+1}^{k} s_{i}(M) \in \mathfrak{M}_{j} \text { for } 0 \leq j \leq k-1,
$$

and $s_{j}(M)$ for $j \geq 1$ has a neighbourhood $V_{j}$ in $M_{j}$ with the structure of a locally trivial $B_{j-1}^{\triangle}$-bundle over $s_{j}(M)$ for some $B_{j-1} \in \mathfrak{M}_{j-1}$. 
In fact, by virtue of Definition 1 (i) we have $M_{k-1}=M \backslash s_{k}(M) \in \mathfrak{M}_{k-1}$. Thus $s_{k-1}(M):=$ $s_{k-1}\left(M_{k-1}\right)$ gives rise to $M_{k-2}:=M_{k-1} \backslash s_{k-1}(M)$, etc. In order to verify (1.6) we identify a neighbourhood of any point of $s_{k}(M)$ with $B_{k-1}^{\Delta} \times \Omega_{k}$ for an open set $\Omega_{k} \subseteq \mathbb{R}^{q_{k}}, q_{k}:=\operatorname{dim} s_{k}(M), B_{k-1}$ $\in \mathfrak{M}_{k-1}$. Then $s_{k-1}(M)$ can be locally identified with $s_{k-1}\left(B_{k-1}^{\Delta}\right) \times \Omega_{k}=s_{k-1}\left(B_{k-1}^{\wedge} \times \Omega_{k}\right)=$ $\mathbb{R}_{+} \times s_{k-1}\left(B_{k-1} \times \Omega_{k}\right)$. Thus

$$
\operatorname{dim} s_{k-1}(M)=1+\operatorname{dim} s_{k-1}\left(B_{k-1}\right)+\operatorname{dim} s_{k}(M)
$$

which yields $\operatorname{dim} s_{k-1}(M)>\operatorname{dim} s_{k}(M)$. Applying the same conclusion to $M_{k-1}$ and then iterating the arguments we obtain the inequalities (1.6).

\subsection{Examples and remarks}

Let us set

$$
\operatorname{dim} M:=\operatorname{dim} s_{0}(M)
$$

\section{Example 1.}

(i) Let $M$ be a smooth manifold with boundary $\partial M$. As in the above-mentioned Zaremba problem we assume that

$$
\partial M=(\partial M)_{-} \cup(\partial M)_{+},(\partial M)_{-} \cap(\partial M)_{+}:=Z,
$$

i.e., the boundary is subdivided into submanifolds $(\partial M)_{ \pm}$with common smooth boundary $Z$ of codimension 1 on $\partial M$. Then we have $M \in \mathfrak{M}_{2}$ and $s_{0}(M)=M \backslash \partial M, s_{1}(M)=$ $\partial M \backslash Z, s_{2}(M)=Z$.

(ii) As noted before a topological space can be stratified in different ways. E.g., we have $M=$ $\mathbb{R}^{n} \in \mathfrak{M}_{0}$. However, if we embed in $\mathbb{R}^{n}$ a smooth hypersurface $S$ of some codimension $\geq 1$, then we have $M=\mathbb{R}^{n} \in \mathfrak{M}_{1}$ and $s_{0}(M)=M \backslash S, s_{1}(M)=S$. In particular, $S$ can be the origin; then $\mathbb{R}^{n}$ becomes a manifold with embedded conical singularity, and we have $M=\mathbb{R}^{n} \in \mathfrak{M}_{1}$.

(iii) For any $B \in \mathfrak{M}_{k-1}$ we have $B^{\triangle} \in \mathfrak{M}_{k}$ and

$$
s_{k}\left(B^{\triangle}\right)=v
$$

with $v$ being the vertex of $B^{\triangle}$ represented by $\{v\} \times B$ in the quotient space in (1.2), and $s_{k-j}\left(B^{\triangle}\right)=\mathbb{R}_{+} \times s_{k-j}(B), j=1, \ldots, k$. Moreover,

$$
B^{\triangle} \times X \in \mathfrak{M}_{k}
$$

for every $X \in \mathfrak{M}_{0}$, and $s_{j}\left(B^{\triangle} \times X\right)=s_{j}\left(B^{\triangle}\right) \times X, j=0, \ldots, k$.

(iv) For $M \in \mathfrak{M}_{k}, k \geq 1$, we have $M \backslash s_{0}(M) \in \mathfrak{M}_{k-1}$. 
Theorem 1. For $M \in \mathfrak{M}_{k}, N \in \mathfrak{M}_{l}$ we have $M \times N \in \mathfrak{M}_{k+l}$.

Let $M \in \mathfrak{M}_{k}$ and define

$$
\text { the stretched space } \quad \mathbb{M} \in \mathfrak{M}_{k} \quad \text { associated with } \quad M
$$

and the double

$$
2 \mathbb{M} \in \mathfrak{M}_{k-1} .
$$

In order to see the meaning we first look at the case $M=B^{\triangle} \times X$ for some $B \in \mathfrak{M}_{k-1}, X \in \mathfrak{M}_{0}$. Then we set

$$
\mathbb{M}:=\overline{\mathbb{R}}_{+} \times B \times X \in \mathfrak{M}_{k} \text { and } 2 \mathbb{M}:=\mathbb{R} \times B \times X \in \mathfrak{M}_{k-1} .
$$

Concerning $M \in \mathfrak{M}_{k}$ in general, from Definition 1 and the subsequent considerations we easily see that the $B$-bundle over $s_{k}(M)$ can be invariantly attached to $M \backslash s_{k}(M)$ which yields $\mathbb{M}$. Recall that the $B$-bundle is at the same time a subbundle of a corresponding $\mathbb{R} \times B$-bundle. Therefore, the construction of attaching that can be performed for another copy of $M \backslash s_{k}(M)$, a negative counterpart of the former space. We then obtain $\mathbb{M}=: \mathbb{M}_{+}$as before and a negative counterpart $\mathbb{M}_{-}$. Both can be glued together along the common $B$-bundle to the double $2 \mathbb{M}$.

\section{Corner-degenerate differential operators}

\subsection{The principal symbolic hierarchies}

In the present section we consider natural (pseudo)-differential operators $A$ on a stratified space $M \in \mathfrak{M}_{k}$ and illustrate the way on how $A$, first given on $s_{0}(M)$ and with a controlled "corner behaviour" close to $M \backslash s_{0}(M)$, acquires from the stratification

$$
s(M)=\left(s_{0}(M), s_{1}(M), \ldots, s_{k}(M)\right)
$$

of $M$ a principal symbolic hierarchy

$$
\sigma(A)=\left(\sigma_{0}(A), \sigma_{1}(A), \ldots, \sigma_{k}(A)\right) .
$$

Other principal symbolic effects appear when the underlying space $M$ has conical exits at infinity. We shall see below, that there is a relationship between operator-valued edge symbols acting on an infinite open stretched cone $X^{\wedge}$ for $X \in \mathfrak{M}_{0}$, and corresponding parameterdependent symbols at the singularity of $X^{\triangle}$ "in the finite". Therefore we also look at the exit behaviour of symbols and operators as a background information on the nature of operatorvalued components of (2.2).

We consider here scalar operators, but the notions easily extend to operators between distributional sections of vector bundles. Let us start with the case of differential operators. 
For $X \in \mathfrak{M}_{0}$ by $\operatorname{Diff}^{\mu}(X)$ we denote the set of all differential operators $A$ on $X$ of order $\mu \epsilon$ $\mathbb{N}, \mu \geq 1$, with smooth coefficients. Then $\sigma_{0}(A)$ is the homogeneous principal symbol of order $\mu$ as an invariantly defined smooth function on $T^{*} X \backslash 0$, the cotangent bundle of $X$ minus zero section, indicated by 0 . For $\mu=0$ we set $\operatorname{Diff}^{0}(X):=C^{\infty}(X)$ and interpret $\sigma_{0}(\cdot)$ as the corresponding function.

Let us first consider some examples and observe structures in special cases. A smooth manifold $M$ with boundary belongs to $\mathfrak{M}_{1}$ and we have

$$
s(M)=\left(s_{0}(M), s_{1}(M)\right) \text { for } \quad s_{0}(M)=M \backslash \partial M, s_{1}(M)=\partial M .
$$

The neighbourhood $V$ in Definition 1 (ii) in this case can be identified with $[0,1) \times \partial M$, a collar neighbourhood of $\partial M$, and the cone bundle is trivial in this case, namely $\overline{\mathbb{R}}_{+} \times \partial M$, the normal bundle of the boundary. We tacitly assume that $M$ is Riemannian and $\partial M$ equipped with the induced Riemannian metric. The space $B \in \mathfrak{M}_{0}$ is a single point. Consider a differential operator $A$ on $M$ of order $\mu$ with smooth coefficients up to the boundary, and write $A$ in local coordinates $x \in \overline{\mathbb{R}}_{+} \times \Omega$ for an open set $\Omega \subseteq \mathbb{R}^{q}$ for $q:=\operatorname{dim} \partial M$ in the form

$$
A=\sum_{|\alpha| \leq \mu} a_{\alpha}(x) D_{x}^{\alpha}
$$

for coefficients $a_{\alpha} \in C^{\infty}\left(\overline{\mathbb{R}}_{+} \times \Omega\right)$. There is then the standard homogeneous principal symbol

$$
\sigma_{0}(A)(x, \xi)=\sum_{|\alpha|=\mu} a_{\alpha}(x) \xi^{\alpha} \quad \text { for } \quad(x, \xi) \in \overline{\mathbb{R}}_{+} \times \Omega \times\left(\mathbb{R}^{n} \backslash\{0\}\right)
$$

In addition we have the homogeneous principal boundary symbol

$$
\sigma_{1}(A)(y, \eta):=\sum_{|\alpha|=\mu, \alpha=\left(\alpha^{\prime}, \alpha^{\prime \prime}\right)} a_{\alpha^{\prime}, \alpha^{\prime \prime}}(0, y) D_{r}^{\alpha^{\prime}} \eta^{\alpha^{\prime \prime}} \quad \text { for } \quad(y, \eta) \in \Omega \times\left(\mathbb{R}^{q} \backslash\{0\}\right)
$$

Here $x=(r, y), \xi=(\rho, \eta), \alpha=\left(\alpha^{\prime}, \alpha^{\prime \prime}\right) \in \mathbb{N} \times \mathbb{N}^{q}, q=n-1$. We can interpret (2.4) as a family of continuous operators

$$
\sigma_{1}(A)(y, \eta): H^{s}\left(\mathbb{R}_{+}\right) \rightarrow H^{s-\mu}\left(\mathbb{R}_{+}\right)
$$

for every $s \in \mathbb{R}$ for $H^{s}\left(\mathbb{R}_{+}\right)=\left.H^{s}(\mathbb{R})\right|_{\mathbb{R}_{+}}$, with $H^{s}(\mathbb{R})$ being the standard Sobolev space of smoothness $s$ on the real axis. For (2.4) homogeneity means

$$
\sigma_{1}(A)(y, \delta \eta)=\delta^{\mu} \kappa_{\delta} \sigma_{1}(A)(y, \eta) \kappa_{\delta}^{-1}
$$

for all $\delta \in \mathbb{R}_{+}$, where

$$
\left(\kappa_{\delta} u\right)(r):=\delta^{1 / 2} u(\delta r), \delta \in \mathbb{R}_{+} .
$$


Remark 2. As is well-known, $\sigma_{0}$-ellipticity of $A$, i.e., $\sigma_{0}(A)(x, \xi) \neq 0$ for all $(x, \xi) \in T^{*} M \backslash 0$, has the consequence that (2.5) is a Fredholm operator for every $(y, \eta) \in \Omega \times\left(\mathbb{R}^{q} \backslash\{0\}\right)$ and $s>\mu-1 / 2$ (even surjective in this case). As far as it concerns the behaviour of operators for $r \rightarrow \infty$, the "conical exit" of the half-axis to infinity, this comes from the ellipticity of subordinate interior and exit symbols for every fixed $(y, \eta) \in \Omega \times\left(\mathbb{R}^{q} \backslash\{0\}\right)$, namely,

$$
\begin{aligned}
\sigma_{\psi}\left(\sigma_{1}(A)(y, \eta)\right)(\rho) & =a_{\mu, 0}(0, y) \rho^{\mu} \neq 0 \text { for all } \rho \in \mathbb{R} \backslash\{0\}, \\
\sigma_{\mathrm{e}}\left(\sigma_{1}(A)(y, \eta)\right)(\rho) & =\sum_{|\alpha|=\mu, \alpha=\left(\alpha^{\prime}, \alpha^{\prime \prime}\right)} a_{\alpha^{\prime}, \alpha^{\prime \prime}}(0, y) \rho^{\alpha^{\prime}} \eta^{\alpha^{\prime \prime}} \neq 0 \text { for all } \rho \in \mathbb{R}, \\
\sigma_{\psi, \mathrm{e}}\left(\sigma_{1}(A)(y, \eta)\right)(\rho) & =a_{\mu, 0}(0, y) \rho^{\mu} \neq 0 \text { for all } \rho \in \mathbb{R} \backslash\{0\} .
\end{aligned}
$$

The boundary symbols of an elliptic operator are responsible for the nature of additional elliptic boundary conditions of trace and potential type, and also for a topological obstruction of Atiyah and Bott, formulated in [4] for the existence of Shapiro-Lopatinskij elliptic conditions for elliptic differential operators.

In the context of the edge symbolic calculus below, we will deepen the impression on the role of conical exits to infinity of a manifold. Let us recall a well-known result.

Let

$$
A=\sum_{|\alpha| \leq \mu} a_{\alpha}(\tilde{x}) D_{\tilde{x}}^{\alpha}
$$

be a differential operator in $\mathbb{R}^{n+1} \ni \tilde{x}$ with coefficients $a_{\alpha}(\tilde{x}) \in S_{\mathrm{cl}}^{0}\left(\mathbb{R}_{\tilde{x}}^{n+1}\right)$, where $\tilde{x}$ formally plays the role of a covariable, and let $a_{\alpha,(0)}(\tilde{x})$ be the homogeneous principal symbol of $a_{\alpha}(\tilde{x})$ of order zero in $\tilde{x} \neq 0$. Set

$$
\begin{aligned}
\sigma_{\psi}(A)(\tilde{x}, \tilde{\xi}) & :=\sum_{|\alpha|=\mu} a_{\alpha}(\tilde{x}) \tilde{\xi}^{\alpha} \quad \text { for } \quad(\tilde{x}, \tilde{\xi}) \in \mathbb{R}^{n+1} \times\left(\mathbb{R}^{n+1} \backslash\{0\}\right), \\
\sigma_{\mathrm{e}}(A)(\tilde{x}, \tilde{\xi}) & :=\sum_{|\alpha| \leq \mu} a_{\alpha,(0)}(\tilde{x}) \tilde{\xi}^{\alpha} \quad \text { for } \quad(\tilde{x}, \tilde{\xi}) \in\left(\mathbb{R}^{n+1} \backslash\{0\}\right) \times \mathbb{R}^{n+1}, \\
\sigma_{\psi, \mathrm{e}}(A)(\tilde{x}, \tilde{\xi}) & :=\sum_{|\alpha|=\mu} a_{\alpha,(0)}(\tilde{x}) \tilde{\xi}^{\alpha} \quad \text { for } \quad(\tilde{x}, \tilde{\xi}) \in\left(\mathbb{R}^{n+1} \backslash\{0\}\right) \times\left(\mathbb{R}^{n+1} \backslash\{0\}\right) .
\end{aligned}
$$

Moreover, let

$$
H^{s ; g}\left(\mathbb{R}^{n+1}\right):=\langle\tilde{x}\rangle^{-g} H^{s}\left(\mathbb{R}^{n+1}\right), \quad s, g \in \mathbb{R}
$$

It can be easily verified that $A: \mathscr{S}\left(\mathbb{R}^{n+1}\right) \rightarrow \mathscr{S}\left(\mathbb{R}^{n+1}\right)$ is continuous and extends to a continuous operator

$$
A: H^{s ; g}\left(\mathbb{R}^{n+1}\right) \rightarrow H^{s-\mu ; g}\left(\mathbb{R}^{n+1}\right)
$$

for every $s, g \in \mathbb{R}$. 
Remark 3. The Euclidean space $\mathbb{R}^{n+1}$ is an example of a manifold with conical exit to infinity $|\tilde{x}| \rightarrow \infty$. The symbols (2.10) illustrate on how the operator (2.9) "acquires" from the conical exit a symbolic structure

$$
\sigma_{\mathrm{E}}(A):=\left(\sigma_{\mathrm{e}}(A), \sigma_{\psi, \mathrm{e}}(A)\right)
$$

here the pair of (principal) exit symbols. The homogeneities are altogether

$$
\begin{aligned}
\sigma_{\psi}(A)(\tilde{x}, \delta \tilde{\xi}) & =\delta^{\mu} \sigma_{\psi}(A)(\tilde{x}, \tilde{\xi}) \quad \text { for all } \quad(\tilde{x}, \tilde{\xi}) \in \mathbb{R}^{n+1} \times\left(\mathbb{R}^{n+1} \backslash\{0\}\right), \\
\sigma_{\mathrm{e}}(A)(\lambda \tilde{x}, \tilde{\xi}) & =\sigma_{\mathrm{e}}(A)(\tilde{x}, \tilde{\xi}) \quad \text { for all } \quad(\tilde{x}, \tilde{\xi}) \in\left(\mathbb{R}^{n+1} \backslash\{0\}\right) \times \mathbb{R}^{n+1}, \\
\sigma_{\psi, \mathrm{e}}(A)(\lambda \tilde{x}, \delta \tilde{\xi}) & =\delta^{\mu} \sigma_{\psi}(A)(\tilde{x}, \tilde{\xi}) \quad \text { for all } \quad(\tilde{x}, \tilde{\xi}) \in\left(\mathbb{R}^{n+1} \backslash\{0\}\right) \times\left(\mathbb{R}^{n+1} \backslash\{0\}\right),
\end{aligned}
$$

and $\lambda, \delta \in \mathbb{R}_{+}$. An adequate compactification of $\mathbb{R}^{n+1}$ in this context is a closed ball $B$, where int $B$ corresponds to $\mathbb{R}^{n+1}$ itself, while $\partial B$ can be identified with the sphere $S^{n}=\{\tilde{x} /|\tilde{x}|: \tilde{x} \neq 0\}$.

The operator (2.9) is called $\left(\sigma_{\psi}, \sigma_{\mathrm{e}}, \sigma_{\psi, \mathrm{e}}\right)$-elliptic if $\sigma_{\psi}(A)(\tilde{x}, \tilde{\xi}) \neq 0$ for all $(\tilde{x}, \tilde{\xi}) \in \mathbb{R}^{n+1} \times$ $\left(\mathbb{R}^{n+1} \backslash\{0\}\right), \sigma_{\mathrm{e}}(A)(\tilde{x}, \tilde{\xi}) \neq 0$ for all $(\tilde{x}, \tilde{\xi}) \in\left(\mathbb{R}^{n+1} \backslash\{0\}\right) \times \mathbb{R}^{n+1}$, and $\sigma_{\psi, \mathrm{e}}(A) \neq 0$ for all $(\tilde{x}, \tilde{\xi}) \in\left(\mathbb{R}^{n+1} \backslash\right.$ $\{0\}) \times\left(\mathbb{R}^{n+1} \backslash\{0\}\right)$.

Theorem 2. The following conditions are equivalent:

(i) $A$ is $\left(\sigma_{\psi}, \sigma_{\mathrm{e}}, \sigma_{\psi, \mathrm{e}}\right)$-elliptic,

(ii) The operator

$$
A: H^{s}\left(\mathbb{R}^{n+1}\right) \rightarrow H^{s-\mu}\left(\mathbb{R}^{n+1}\right)
$$

is Fredholm for every $s \in \mathbb{R}$.

Let us now turn to natural differential operators on a space $M \in \mathfrak{M}_{k}$. Those will be expressed locally close to $s_{k}(M)$ in "stretched variables". As such they will be degenerate in a similar way as operators in polar or cylindrical coordinates that are originally smooth across an embedded conical or edge singularity.

A differential operator

$$
A=\sum_{|\alpha| \leq \mu} a_{\alpha}(\tilde{x}) D_{\tilde{x}}^{\alpha}
$$

of order $\mu \in \mathbb{N}$ with smooth coefficients in $\mathbb{R}^{n+1} \ni \tilde{x}$ in polar coordinates $(r, x) \in \mathbb{R}_{+} \times S^{n}$ for $\tilde{x} \neq 0$ takes the form

$$
A=r^{-\mu} \sum_{j=0}^{\mu} a_{j}(r)\left(-r \frac{\partial}{\partial r}\right)^{j}
$$

for coefficients $a_{j}(r) \in C^{\infty}\left(\overline{\mathbb{R}}_{+}\right.$, Diff $\left.{ }^{\mu-j}(X)\right)$ and $X=S^{n}$ in this case. The minus sign at the Fuchs type derivative $r \partial_{r}$ is motivated by the Mellin transform. 
More generally, an operator of order $\mu$ with smooth coefficients in $\mathbb{R}^{n+1} \times \mathbb{R}^{q} \ni(\tilde{x}, y), q>0$,

$$
A=\sum_{|\alpha| \leq \mu} a_{\alpha}(\tilde{x}, y) D_{\tilde{x}, y}^{\alpha}
$$

in polar coordinates for $\tilde{x} \neq 0$ becomes edge-degenerate, i.e.,

$$
A=r^{-\mu} \sum_{j+|\alpha| \leq \mu} a_{j \alpha}(r, y)\left(-r \frac{\partial}{\partial r}\right)^{j}\left(r D_{y}\right)^{\alpha}
$$

for coefficients $a_{j \alpha}(r, y) \in C^{\infty}\left(\overline{\mathbb{R}}_{+} \times \mathbb{R}^{q}, \operatorname{Diff}^{\mu-(j+|\alpha|)}(X)\right), X=S^{n}$.

\section{Example 2.}

(i) The Laplacian

$$
\Delta=\sum_{j=1}^{n+1} \frac{\partial^{2}}{\partial \tilde{x}_{j}^{2}}
$$

in $\mathbb{R}^{n+1}$ in polar coordinates $(r, x) \in \mathbb{R}_{+} \times S^{n}$ in $\mathbb{R}^{n+1} \backslash\{0\}$ has the form

$$
\Delta=r^{-2}\left(\left(r \frac{\partial}{\partial r}\right)^{2}+(n-1) r \frac{\partial}{\partial r}+\Delta_{S^{n}}\right)
$$

where $\Delta_{S^{n}}$ is the Laplace operator on $S^{n}$.

(ii) The operator

$$
\sum_{j=1}^{n+1} \tilde{x}_{j} \frac{\partial}{\partial \tilde{x}_{j}}
$$

in polar coordinates takes the form

$$
r \frac{\partial}{\partial r}
$$

(iii) The Laplace-Beltrami operator belonging to the Riemannian metric

$$
d r^{2}+r^{2} g_{X}+d y^{2}
$$

on $\mathbb{R}_{+} \times X \times \mathbb{R}^{q}$, where $g_{X}$ is a Riemannian metric on $X$ has the form

$$
r^{-2}\left(\sum_{j=0}^{2} a_{j}(r)\left(r \frac{\partial}{\partial r}\right)^{j}+\sum_{l=1}^{q}\left(r \frac{\partial}{\partial y_{l}}\right)^{2}\right)
$$

for coefficients $a_{j}(r) \in C^{\infty}\left(\overline{\mathbb{R}}_{+}, \operatorname{Diff}^{2-j}(X)\right)$.

Definition 2. Let $M \in \mathfrak{M}_{k}, k \geq 1$.

(i) For $\operatorname{dim} s_{k}(M)=0$ the space $\operatorname{Diff}_{\operatorname{deg}}^{\mu}(M), \mu \in \mathbb{N}$, is defined as the set of all $A \in \operatorname{Diff}_{\operatorname{deg}}^{\mu}(M \backslash$ $\left.s_{k}(M)\right)$ that are close to $s_{k}(M)$ in the splitting of variables $(r, \cdot) \in \mathbb{R}_{+} \times X, X \in \mathfrak{M}_{k-1}$, of the form

$$
A=r^{-\mu} \sum_{j=0}^{\mu} a_{j}(r)\left(-r \frac{\partial}{\partial r}\right)^{j},
$$

for coefficients $a_{j}(r) \in C^{\infty}\left(\overline{\mathbb{R}}_{+}, \operatorname{Diff}_{\mathrm{deg}}^{\mu-j}(X)\right)$. 
(ii) For $\operatorname{dim} s_{k}(M)=: q>0$ the space $\operatorname{Diff}_{\mathrm{deg}}^{\mu}(M), \mu \in \mathbb{N}$, is defined as the set of all $A \in \operatorname{Diff}_{\mathrm{deg}}^{\mu}\left(M \backslash s_{k}(M)\right)$ that are close to $s_{k}(M)$ in the splitting of variables $(r, \cdot, y) \in \mathbb{R}_{+} \times X \times \Omega, X \in \mathfrak{M}_{k-1}, \Omega \subseteq \mathbb{R}^{q}$, of the form

$$
A=r^{-\mu} \sum_{j+|\alpha| \leq \mu} a_{j \alpha}(r, y)\left(-r \frac{\partial}{\partial r}\right)^{j}\left(r D_{y}\right)^{\alpha},
$$

for coefficients $a_{j \alpha}(r, y) \in C^{\infty}\left(\overline{\mathbb{R}}_{+} \times \Omega\right.$, Diff $\left.\operatorname{deg}^{\mu-j}(X)\right)$.

We now define

$$
\sigma_{k}(A)(z)=\sum_{j=0}^{\mu} a_{j}(0) z^{j}
$$

for $\operatorname{dim} s_{k}(M)=0, z \in \Gamma_{\beta}(=\{z \in \mathbb{C}: \operatorname{Re} z=\beta\}$ for some $\beta \in \mathbb{R})$, and

$$
\sigma_{k}(A)(y, \eta)=r^{-\mu} \sum_{j+|\alpha| \leq \mu} a_{j \alpha}(0, y)\left(-r \frac{\partial}{\partial r}\right)^{j}(r \eta)^{\alpha}
$$

for $\operatorname{dim} s_{k}(M)>0,(y, \eta) \in T^{*} \Omega \backslash 0$. The symbol $\sigma_{k}(A)(z)$ takes values in $\operatorname{Diff} f_{\operatorname{deg}}^{\mu}(X)$ for $\operatorname{dim} s_{k}(M)$ $=0$, and in Diff ${ }_{\mathrm{deg}}^{\mu}\left(X^{\wedge}\right)$ for $\operatorname{dim} s_{k}(M)>0$, cf. (1.6).

By virtue of

$$
\operatorname{Diff}_{\mathrm{deg}}^{\mu}(M) \subseteq \operatorname{Diff}_{\mathrm{deg}}^{\mu}\left(M \backslash s_{k}(M)\right)
$$

we can determine also $\sigma_{k-1}(A)$ with respect to $s_{k-1}(M)$ which is of dimension $>0$, cf. (1.6). For $k \geq 2$ we can continue this construction, and we finally obtain $k+1$ components of $\sigma(A)$, namely, (2.2), where $\sigma_{0}(A) \in C^{\infty}\left(T^{*}\right.$ (int $\left.M\right) \backslash 0$ ) is the standard homogeneous principal symbol of $A$ on $s_{0}(M)$. The other components $\sigma_{j}(A), j>0$, are operator-valued and associated with $s_{j}(M)$. They are of analogous form as (2.24), parametrised by $(y, \eta) \in T^{*} s_{j}(M) \backslash 0$, and take values in $\operatorname{Diff}_{\mathrm{deg}}^{\mu}\left(B_{j-1}^{\Delta}\right)$, cf. notation after Remark 1 for $0<j<k$. As such they define families of continuous operators

$$
\sigma_{j}(A)(y, \eta): C_{0}^{\infty}\left(s_{0}\left(B_{j-1}^{\triangle}\right)\right) \rightarrow C_{0}^{\infty}\left(s_{0}\left(B_{j-1}^{\triangle}\right)\right) .
$$

Let us set

$$
\left(\iota_{\delta} u\right)(r, \cdot):=u(\delta r, \cdot), \delta \in \mathbb{R}_{+}
$$

which defines a group $\iota:=\left\{l_{\delta}\right\}_{\delta \in \mathbb{R}_{+}}$of isomorphisms

$$
\iota_{\delta}: C_{0}^{\infty}\left(s_{0}\left(B_{j-1}^{\triangle}\right)\right) \rightarrow C_{0}^{\infty}\left(s_{0}\left(B_{j-1}^{\triangle}\right)\right) .
$$

The symbols (2.25) are homogeneous in the sense

$$
\sigma_{j}(A)(y, \delta \eta)=\delta^{\mu} \iota_{\delta} \sigma_{j}(A)(y, \eta) \iota_{\delta}^{-1}, \delta \in \mathbb{R}_{+},
$$

also referred to as twisted homogeneity of order $\mu$. 
Remark 4. In the well-known edge pseudo-differential calculus, created in [42], for $B \in \mathfrak{M}_{0}$ it is common to take the group $\kappa=\left\{\kappa_{\delta}\right\}_{\delta \in \mathbb{R}_{+}}$, defined by $\kappa_{\delta}:=\delta^{\frac{\operatorname{dim} B^{\Delta}}{2}} \iota_{\delta}$. Such a modification does not change the meaning of homogeneity. However, in pseudo-differential theories with twisted symbolic estimates a suitable additional power of $\delta$ makes sense, and this will be also the case for higher singularities. Moreover, the involved distribution spaces are affected by the choice of the $\delta$-power. For instance, if $B \in \mathfrak{M}_{0}$ is compact, then $\kappa$ is a group of unitary operators in $L^{2}\left(B^{\triangle}\right):=r^{-\frac{\operatorname{dim} B}{2}} L^{2}\left(B^{\wedge}\right)$.

Remark 5. Let $A \in \operatorname{Diff}_{\operatorname{deg}}^{\mu}(M), \tilde{A} \in \operatorname{Diff}_{\operatorname{deg}}^{\tilde{\mu}}(M)$; then $A \tilde{A} \in \operatorname{Diff}_{\operatorname{deg}}^{\mu+\tilde{\mu}}(M)$, and we have $\sigma(A \tilde{A})=$ $\sigma(A) \sigma(\tilde{A})$, with componentwise composition and the rule $\sigma_{k}(A \tilde{A})=T^{\tilde{\mu}} \sigma(A) \sigma(\tilde{A})$ when $\operatorname{dim} s_{k}(M)=0$ for $\left(T^{\beta} f\right)(z):=f(z+\beta)$ for $\beta \in \mathbb{R}$.

\subsection{Fourier and Mellin representations of differential operators}

After having introduced typical differential operators on a singular manifold $M \in \mathfrak{M}_{k}$, it is an obvious question on how to formulate spaces of pseudo-differential operators $L^{\mu}(M)$ containing $\operatorname{Diff}_{\mathrm{deg}}^{\mu}(M)$ for $\mu \in \mathbb{N}$, such that $\bigcup_{\mu} L^{\mu}(M)$ is an algebra which is closed under the construction of parametrices of elliptic elements. Such a program has many aspects, and the details, even in the case of a manifold with conical or edge singularities, show that a notion $L^{\mu}(M)$ is loaded with other data, e.g., weights, or extra trace and potential data along the strata $s_{j}(M), 0<j \leq k$, equipped with symbols, likewise involved in the ellipticity. In particular, operators in $L^{\mu}(M)$ should consist of block matrices that integrate potential and trace operators, similarly as in boundary value problems, e.g., for the Laplacian with Dirichlet or Neumann conditions. Throughout this discussion the notion "algebra" is used here in the sense that algebraic operations are possible within the structure when the operators fit together, e.g., rows and columns in the middle of matrix compositions.

The operators in such algebras are to be designed in such a way that they induce continuous operators in natural distribution spaces. While differential operators essentially act "as they are", i.e., by local differentiations, pseudo-differential operators have to be specified by quantisations (operator conventions) that modify the operators in "naive" representation via Fourier or Mellin transform by some smoothing operators in order to achieve an expected continuity property, say, in weighted Sobolev spaces, or subspaces with asymptotics. In addition, besides differential operators there can appear other operators, contributed by the strata, either in compositions or parametrix constructions, such as ideals of smoothing Mellin plus Green operators, which are in general non-compact. Those have a non-trivial symbolic structure, and also the Fredholm index of elliptic operators can be affected by such contributions. Some phenomena of that kind can be illustrated in the framework of quantisations of (in general, operator-valued) symbols. 
An operator $A \in \operatorname{Diff}_{\mathrm{deg}}^{\mu}(M)$ for $\operatorname{dim} s_{k}(M)=0$, cf. (2.21), can be written as a Fourier (pseudo-)differential operator on the $r$ half-axis $\mathbb{R}_{+}$

$$
A u(r)=\mathrm{Op}_{r}(p) u(r)=r^{-\mu} \iint e^{i\left(r-r^{\prime}\right) \rho} p(r, \rho) u\left(r^{\prime}\right) d r^{\prime} d \rho
$$

for an operator-valued symbol

$$
p(r, \rho)=\tilde{p}(r, r \rho) \quad \text { for } \quad \tilde{p}(r, \tilde{\rho}) \in C^{\infty}\left(\overline{\mathbb{R}}_{+}, \operatorname{Diff}_{\mathrm{deg}}^{\mu}\left(X ; \mathbb{R}_{\tilde{\rho}}\right)\right) .
$$

Here $C^{\infty}\left(\overline{\mathbb{R}}_{+}, \operatorname{Diff}_{\operatorname{deg}}^{\mu}\left(X ; \mathbb{R}_{\tilde{\rho}}\right)\right)$ is the set of all operator functions of the form

$$
\tilde{p}(r, \tilde{\rho}):=\sum_{j=0}^{\mu} a_{j}(r)(-i \tilde{\rho})^{j},
$$

for coefficients $a_{j}(r) \in C^{\infty}\left(\overline{\mathbb{R}}_{+}\right.$, Diff $\left.{ }_{\text {deg }}^{\mu-j}(X)\right)$. In (2.29) as argument functions we can take for the moment $u(r) \in C_{0}^{\infty}\left(\mathbb{R}_{+}, C_{0}^{\infty}(K)\right)$ for any $K \Subset s_{0}(X), C_{0}^{\infty}(K)=\left\{u \in C_{0}^{\infty}\left(s_{0}(X)\right)\right.$ : supp $\left.u \subseteq K\right\}$.

Another useful representation refers to the Mellin transform on the half-axis

$$
M u(z):=\int_{0}^{\infty} r^{z} u(r) \frac{d r}{r},
$$

where $u(r) \in C_{0}^{\infty}\left(\mathbb{R}_{+}, C_{0}^{\infty}(K)\right)$ entails $M u(z) \in \mathscr{A}\left(\mathbb{C}, C_{0}^{\infty}(K)\right)$ and $\left.M u(z)\right|_{\Gamma_{\beta}} \in \mathscr{S}\left(\Gamma_{\beta}, C_{0}^{\infty}(K)\right)$ for any real $\beta$. Here

$$
\Gamma_{\beta}:=\{z \in \mathbb{C}: \operatorname{Re} z=\beta\} .
$$

Distribution spaces on $\Gamma_{\beta}$ such as the Schwartz space $\mathscr{S}\left(\Gamma_{\beta}, E\right)$ with values in a Fréchet space $E$ are interpreted as spaces defined as pull back of corresponding spaces referring to $\mathbb{R}$ under the map $\Gamma_{\beta} \rightarrow \mathbb{R}, z \mapsto \operatorname{Im} z$. Other examples in that sense are $L^{2}\left(\Gamma_{\beta}\right)$, or spaces of pseudodifferential symbols

$$
S^{\mu}\left(\mathbb{R}_{+} \times \Omega \times \Gamma_{\beta} \times \mathbb{R}^{q}\right)
$$

of order $\mu$, depending on variables $(r, y) \in \mathbb{R}_{+} \times \Omega$ and covariables $(z, \eta) \in \Gamma_{\beta} \times \mathbb{R}^{q}$. The motivation for such a notation is that we distinguish spaces for different $\beta, \beta^{\prime}$. Another notation in this context is that we say that a family $f_{\beta}$ of elements, for instance, in $\mathscr{S}\left(\Gamma_{\beta}, E\right)$, uniformly belongs to that space for $c \leq \beta \leq c^{\prime}$, if that family is bounded in $\mathscr{S}(\mathbb{R}, E)$, under the identification of the latter space with $\mathscr{S}\left(\Gamma_{\beta}, E\right)$. For instance, the property

$$
\left.M u(z)\right|_{\Gamma_{\beta}} \in \mathscr{S}\left(\Gamma_{\beta}, C_{0}^{\infty}(K)\right)
$$

for $u(r) \in C_{0}^{\infty}\left(\mathbb{R}_{+}, C_{0}^{\infty}(K)\right)$ is uniform in compact $\beta$-intervals.

Let us call

$$
M_{\gamma} u:=\left.M u\right|_{\Gamma_{1 / 2-\gamma}}
$$


the weighted Mellin transform with weight $\gamma \in \mathbb{R}$. Recall that

$$
M_{\gamma}^{-1} g(r)=\int_{\Gamma_{\frac{1}{2}-\gamma}} r^{-z} g(z) d z
$$

$\varpi z=(2 \pi i)^{-1} d z$. Weighted Mellin pseudo-differential operators are defined as

$$
\mathrm{Op}_{M}^{\gamma}(f) u(r):=M_{\gamma}^{-1}\left(f(r, z) M_{\gamma} u(z)\right)
$$

for Mellin symbols $f(r, z) \in S^{\mu}\left(\mathbb{R}_{+} \times \Gamma_{1 / 2-\gamma}\right)$, later on also for $f\left(r, r^{\prime}, z\right) \in S^{\mu}\left(\mathbb{R}_{+} \times \mathbb{R}_{+} \times \Gamma_{1 / 2-\gamma}\right)$, or more general, also operator-valued symbols. Note that

$$
\mathrm{Op}_{M}^{\gamma}(f):=r^{\gamma} \mathrm{Op}_{M}\left(T^{-\gamma} f\right) r^{-\gamma}
$$

for $\left(T^{\beta} f\right)(z):=f(z+\beta)$ for any $\beta \in \mathbb{R}$ and $\mathrm{Op}_{M}:=\mathrm{Op}_{M}^{0}$, or

$$
\mathrm{Op}_{M}^{\gamma}(f) u(r):=\iint\left(\frac{r}{r^{\prime}}\right)^{-(1 / 2-\gamma+i \rho)} f(r, 1 / 2-\gamma+i \rho) u\left(r^{\prime}\right) \frac{d r^{\prime}}{r^{\prime}} d \rho .
$$

We apply Mellin operators for symbols of the form

$$
f(r, z):=\sum_{j=0}^{\mu} a_{j}(r) z^{j}
$$

with the same coefficients $a_{j}(r) \in C^{\infty}\left(\overline{\mathbb{R}}_{+}, \operatorname{Diff}_{\mathrm{deg}}^{\mu-j}(X)\right)$ as in (2.31). For (2.29) we then obtain

$$
A=\mathrm{Op}_{r}(p)=\mathrm{Op}_{M}^{\gamma}(f)
$$

regarded as a map

$$
A: C_{0}^{\infty}\left(\mathbb{R}_{+}, C_{0}^{\infty}(K)\right) \rightarrow C_{0}^{\infty}\left(\mathbb{R}_{+}, C_{0}^{\infty}(K)\right)
$$

for any $K \Subset s_{0}(X)$. Because of the holomorphic dependence of $f$ on $z$ the choice of the weight $\gamma$ is not essential. However, as soon as we want to extend (2.41) to weighted Sobolev spaces, the weight becomes important. The symbol $\sigma_{k}(A)(z)$, cf. (2.29), is equal to

$$
\sigma_{k}(A)(z)=f(0, z)
$$

Assuming for the moment $\gamma=1 / 2$ the relationship between

$$
f(r, z) \text { and } \quad p(r, \rho)=\tilde{p}(r, r \rho)
$$

involved in (2.40) can be illustrated by the correspondence between $z$ and $-\operatorname{ir} \rho=: b(r, \rho)$, where

$$
-r \frac{\partial}{\partial r}=\mathrm{Op}_{M}^{1 / 2}(z)=\mathrm{Op}_{r}(b)
$$


This yields

$$
\begin{aligned}
\mathrm{Op}_{M}^{1 / 2}(f) & =\sum_{j=0}^{\mu} a_{j}(r) \mathrm{Op}_{M}^{1 / 2}\left(z^{j}\right) \\
& =\sum_{j=0}^{\mu} a_{j}(r)\left(\mathrm{Op}_{r}(b)\right)^{j}=\sum_{j=0}^{\mu} a_{j}(r) \mathrm{Op}_{r}\left(b^{\# j}\right)
\end{aligned}
$$

for $b^{\# j}:=b \#_{r} b \#_{r} \ldots \#_{r} b$ with $j$ factors of the Leibniz product between symbols $b(r, \rho)$, in the composition of Fourier pseudo-differential operators. In other words,

$$
p(r, \rho)=\sum_{j=0}^{\mu} a_{j}(r)(-i r \rho)^{\# j} .
$$

Note that in this case the Leibniz products are "exact", i.e., finite sums, since the factors are polynomials in the covariable $\rho$.

Remark 6. For every $n \in \mathbb{N}$ there are numbers $s_{n, k}, S_{n, k}$ such that

$$
r^{n} \partial_{r}^{n}=\sum_{k=0}^{n} s_{n, k}\left(r \partial_{r}\right)^{k},\left(r \partial_{r}\right)^{n}=\sum_{k=0}^{n} S_{n, k} r^{k} \partial_{r}^{k} .
$$

The numbers $s_{n, k}$ and $S_{n, k}$ are known as the Stirling numbers of first and second kind, respectively. More details may be found in [16, Section 2.2.1].

The symbol $\sigma_{k}(A)$ in the terminology of (2.23) is

$$
\sigma_{k}(A)(z)=f(0, z)
$$

Let us now do similar things for operators $A \in \operatorname{Diff}_{\operatorname{deg}}^{\mu}(M), \operatorname{dim} s_{k}(M)=q>0$, cf. Definition 2 (ii). An operator (2.22) can be written as a Fourier (pseudo-) differential operator on the $r$ half-axis $\mathbb{R}_{+}$

$$
A u(r, y)=\mathrm{Op}_{y, r}(p) u(r, y)=r^{-\mu} \iint e^{i\left(r-r^{\prime}\right) \rho+i\left(y-y^{\prime}\right) \eta} p(r, y, \rho, \eta) u\left(r^{\prime}, y^{\prime}\right) d r^{\prime} d y^{\prime} d \rho d \eta
$$

for an operator-valued symbol

$$
p(r, y, \rho, \eta)=\tilde{p}(r, y, r \rho, r \eta) \quad \text { for } \quad \tilde{p}(r, y, \tilde{\rho}, \tilde{\eta}) \in C^{\infty}\left(\overline{\mathbb{R}}_{+} \times \Omega, \operatorname{Diff}_{\operatorname{deg}}^{\mu}\left(X ; \mathbb{R}_{\tilde{\rho}, \tilde{\eta}}^{1+q}\right)\right)
$$

From (2.48) we pass to another operator-valued symbol

$$
\mathrm{Op}_{r}\left(r^{-\mu} p\right)(y, \eta) \in S^{\mu}\left(\Omega \times \mathbb{R}^{q} ; C_{0}^{\infty}\left(K^{\wedge}\right), C_{0}^{\infty}\left(K^{\wedge}\right)\right)
$$

for $K^{\wedge}=\mathbb{R}_{+} \times K, K \Subset s_{0}(X)$. We then have

$$
A u(\cdot, y)=\mathrm{Op}_{y}\left(\mathrm{Op}_{r}\left(r^{-\mu} p\right)(y, \eta)\right) u(\cdot, y)=\iint e^{i\left(y-y^{\prime}\right) \eta} \mathrm{Op}_{r}\left(r^{-\mu} p\right)(y, \eta) u\left(\cdot, y^{\prime}\right) d y^{\prime} d \eta
$$


Analogously as in boundary value problems we now form a higher principal edge symbol, in fact, another representation of (2.24),

$$
\sigma_{k}(A)(y, \eta)=\operatorname{Op}_{r}\left(r^{-\mu} p_{0}\right)(y, \eta) \in S^{(\mu)}\left(\Omega \times\left(\mathbb{R}^{q} \backslash\{0\}\right) ; C_{0}^{\infty}\left(K^{\wedge}\right), C_{0}^{\infty}\left(K^{\wedge}\right)\right)
$$

for

$$
p_{0}(r, y, \rho, \eta):=\tilde{p}(0, y, r \rho, r \eta)
$$

Applying Mellin operators for symbols

$$
f(r, y, z, \eta)=\tilde{f}(r, y, z, r \eta) \quad \text { for } \quad \tilde{f}(r, y, z, \tilde{\eta}):=\sum_{j+|\alpha| \leq \mu} a_{j \alpha}(r, y) z^{j} \tilde{\eta}^{\alpha},
$$

it follows that

$$
A=\mathrm{Op}_{y}\left(\mathrm{Op}_{M_{r}}^{\gamma}\left(r^{-\mu} f\right)(y, \eta)\right)
$$

i.e.,

$$
A u(r, y)=\iint\left(\frac{r}{r^{\prime}}\right)^{-(1 / 2-\gamma+i \rho)} e^{i\left(y-y^{\prime}\right) \eta} f(r, y, 1 / 2-\gamma+i \rho, \eta) u\left(r^{\prime}, y^{\prime}\right) \frac{d r^{\prime}}{r^{\prime}} d \rho d y^{\prime} d \eta,
$$

The edge symbol in this case can be written

$$
\sigma_{k}(A)(y, \eta)=\operatorname{Op}_{M_{r}}^{\gamma}\left(r^{-\mu} f_{0}\right)(y, \eta)
$$

for

$$
f_{0}(r, y, z, \eta):=\tilde{f}(0, y, z, r \eta)
$$

Let us point out that both in (2.47) with (2.48) and in (2.54) with the symbol (2.53) the involved $r$-variable is treated as a left variable, i.e., contained as a multiplication operator by $r$ from the left. Clearly there are also right symbols where $r$ under the oscillatory integral turns to $r^{\prime}$, but for the moment we ignore such a representation. For $\gamma=1 / 2$ the relationship between

$$
f(r, y, z, \eta)=\tilde{f}(r, y, z, r \eta) \quad \text { and } \quad p(r, y, \rho, \eta)=\tilde{p}(r, y, r \rho, r \eta)
$$

involved in (2.47) follows from

$$
\begin{aligned}
\operatorname{Op}_{y}\left(\mathrm{Op}_{M_{r}}^{1 / 2}(f)(y, \eta)\right) & =\sum_{j+|\alpha| \leq \mu} a_{j \alpha}(r, y) \mathrm{Op}_{M_{r}}^{1 / 2}\left((z)^{j}\right) \mathrm{Op}_{y}\left((r \eta)^{\alpha}\right) \\
& =\sum_{j+|\alpha| \leq \mu} a_{j \alpha}(r, y) \mathrm{Op}_{r}((-i r \rho))^{j} \mathrm{Op}_{y}\left((r \eta)^{\alpha}\right),
\end{aligned}
$$

i.e., we obtain

$$
p(r, y, \rho, \eta)=\sum_{j+|\alpha| \leq \mu} a_{j \alpha}(r, y)(-i r \rho)^{\# j}(r \eta)^{\alpha} .
$$




\subsection{Mellin pseudo-differential operators}

Consider pseudo-differentialoperators based on the weighted Mellin transform $M_{\gamma}$

$$
A u(r)=\iint\left(\frac{r}{r^{\prime}}\right)^{-(1 / 2-\gamma+i \rho)} f\left(r, r^{\prime}, 1 / 2-\gamma+i \rho\right) u\left(r^{\prime}\right) \frac{d r^{\prime}}{r^{\prime}} d \rho,
$$

cf. also formula (2.55). We shall see that there are many choices of amplitude functions. In particular, we may have $f\left(r, r^{\prime}, z\right) \in L_{(\mathrm{cl})}^{\mu}\left(X ; \Gamma_{1 / 2-\gamma}\right)$ for a closed smooth manifold $X$. The elements of the pseudo-differential calculus based on the Fourier transform have analogues for Mellin pseudo-differential operators. In particular, every operator (2.60) can be written as a properly supported operator, modulo a smoothing one.

Remark 7. For $\left(\delta_{\lambda} u\right)(r):=u(\lambda r), \lambda>0$, and a Mellin amplitude function $f\left(r, r^{\prime}, z\right)$ we have

$$
\delta_{\lambda} \mathrm{Op}_{M}^{\gamma}(f) \delta_{\lambda}^{-1}=\mathrm{Op}_{M}^{\gamma}\left(f_{\lambda}\right)
$$

for $f_{\lambda}\left(r, r^{\prime}, z\right)=f\left(\lambda r, \lambda r^{\prime}, z\right)$.

Remark 8. By virtue of $M_{\gamma} u\left(\frac{1}{2}-\gamma+i \rho\right)=\int r^{\left(\frac{1}{2}-\gamma+i \rho\right)} u(r) d r / r$ and $u(r)=\int r^{-\left(\frac{1}{2}-\gamma+i \rho\right)} M_{\gamma} u\left(\frac{1}{2}-\right.$ $\gamma+i \rho) d \rho$ for a properly supported operator $A=\mathrm{Op}_{M}^{\gamma}(f)$ we have

$$
A u(r)=\int r^{-\left(\frac{1}{2}-\gamma+i \rho\right)}\left(r^{\left(\frac{1}{2}-\gamma+i \rho\right)} A r^{-\left(\frac{1}{2}-\gamma+i \rho\right)}\right) M_{\gamma} u\left(\frac{1}{2}-\gamma+i \rho\right) d \rho .
$$

Thus

$$
f_{L}:=r^{\left(\frac{1}{2}-\gamma+i \rho\right)} A r^{-\left(\frac{1}{2}-\gamma+i \rho\right)}
$$

is a left Mellin symbol of $A$.

\section{Kernel cut-off and Mellin quantization}

\subsection{Kernel cut-off based on the Fourier transform}

Pseudo-differential operators on spaces $M \in \mathfrak{M}_{1}$, i.e., with conical or edge singularities, contain symbols that are holomorphic in the covariable $z \in \mathbb{C}$ belonging to the Mellin transform on $\mathbb{R}_{+}$. We saw in Subsection 2.2 that differential operators $A \in \operatorname{Diff}_{\operatorname{deg}}^{\mu}(M)$ on $M \in \mathfrak{M}_{k}$ locally close to $s_{k}(M)$ can be expressed both in terms of the Fourier and the Mellin transform. We now deepen the relations between Fourier and Mellin symbols of degenerate pseudodifferential operators. In addition we study here kernel cut-off which creates holomorphic symbols with remarkable properties. Kernel cut-off is a generic term for systems of operators acting on symbols. The method has been introduced in [41] and later on widely applied in the analysis of operators on manifolds with conical or edge singularities, cf. [43] or [45]. In this 
subsection we refer to technical details on the kernel cut-off technique, contributed by Seiler [54] and Krainer [31].

Let us start with a definition of kernel cut-off in connection with the Fourier transform. In order to motivate the construction we first consider a scalar symbol with constant coefficients

$$
a(\xi) \in S_{(\mathrm{cl})}^{\mu}\left(\mathbb{R}^{n}\right)
$$

The associated pseudo-differential operator

$$
\mathrm{Op}(a) u(x)=\iint e^{i\left(x-x^{\prime}\right) \xi} a(\xi) u\left(x^{\prime}\right) d x^{\prime} d \xi
$$

in $\mathbb{R}^{n}$ has the distributional kernel $k(a)\left(x-x^{\prime}\right)$ for

$$
k(a)(\theta):=\int e^{i \theta \xi} a(\xi) d \xi .
$$

In (3.2) we interpret $a(\xi)$ as an element of $\mathscr{S}^{\prime}\left(\mathbb{R}_{\xi}^{n}\right)$; then $k(a)(\theta) \in \mathscr{S}^{\prime}\left(\mathbb{R}_{\theta}^{n}\right)$. We often refer to the following standard properties of the Fourier transform:

$$
(-\theta)^{\alpha} k(a)(\theta)=k\left(D_{\xi}^{\alpha} a\right)(\theta), D_{\theta}^{\alpha} k(a)(\theta)=k\left(\xi^{\alpha} a\right)(\theta), \quad \alpha \in \mathbb{N}^{n} .
$$

Lemma 1. We have

$$
\chi(\theta) k(a)(\theta) \in \mathscr{S}\left(\mathbb{R}_{\theta}^{n}\right)
$$

for every excision function $\chi(\theta)$ in $\mathbb{R}^{n}$.

Proof. We employ the identities

$$
|\theta|^{2 N} e^{i \theta \xi}=(-1)^{N} \Delta_{\xi}^{N} e^{i \theta \xi}, \Delta_{\theta}^{M} e^{i \theta \xi}=(-1)^{M}|\xi|^{2 M} e^{i \theta \xi}
$$

for every $N, M \in \mathbb{N}$. This yields after integration by parts

as well as

$$
\begin{aligned}
\chi(\theta)|\theta|^{2 N} \Delta_{\theta}^{M} k(a)(\theta) & =\chi(\theta)|\theta|^{2 N}(-1)^{M} \int e^{i \theta \xi}|\xi|^{2 M} a(\xi) d \xi \\
& =(-1)^{(N+M)} \chi(\theta) \int e^{i \theta \xi} \Delta_{\xi}^{N}\left(|\xi|^{2 M} a(\xi)\right) d \xi
\end{aligned}
$$

$$
\left.\chi(\theta) k(a)(\theta)=\chi(\theta)(-1)^{N}|\theta|^{-2 N} \int e^{i \theta \xi} \Delta_{\xi}^{N} a(\xi)\right) d \xi
$$

which follows from the second equation of (3.5) for $M=0$. Since (3.6) holds for an arbitrary excision function $\chi$ and every $N$ from $\left.\Delta_{\xi}^{N} a(\xi)\right) \in S^{\mu-2 N}\left(\mathbb{R}^{n}\right)$ we conclude $k(a)(\theta) \in C^{\infty}\left(\mathbb{R}^{n} \backslash\{0\}\right)$, in particular, sing supp $k(a) \subseteq\{0\}$. This is equivalent to the pseudo-locality of the operator $\operatorname{Op}(a)$, i.e., the singular support of its distributional kernel is contained in $\operatorname{diag}\left(\mathbb{R}^{n} \times \mathbb{R}^{n}\right)$. Therefore, in order to verify (3.4) it suffices so show that

$$
\sup _{|\theta| \geq C} \|\left.\theta\right|^{2 N} \Delta_{\theta}^{M} k(a)(\theta) \mid
$$


is bounded for some $C>0$ and suitable sufficiently large $M, N$. But this follows from the absolute convergence of the integrals on the right hand side of (3.5) for arbitrary $M$ by choosing $N$ so large that $\mu+2(M-N)<-n$.

Let

$$
\psi(\theta):=1-\chi(\theta)
$$

which is a cut-off function, i.e., $\psi \in C_{0}^{\infty}\left(\mathbb{R}^{n}\right), \psi(\theta) \equiv 1$ in a neighbourhood of $\theta=0$. We call

$$
V_{F}(\psi) a(\xi):=F_{\theta \rightarrow \xi}(\psi k(a))(\xi)
$$

a kernel cut-off operator and $V_{F}(\chi):=F_{\theta \rightarrow \xi}(\chi k(a))(\xi)$ a kernel excision operator.

Proposition 1. The operator (3.7) induces a continuous map

$$
V_{F}(\psi): S_{(\mathrm{cl})}^{\mu}\left(\mathbb{R}^{n}\right) \rightarrow S_{(\mathrm{cl})}^{\mu}\left(\mathbb{R}^{n}\right)
$$

where

$$
V_{F}(\psi) a(\xi)=a(\xi) \bmod S^{-\infty}\left(\mathbb{R}^{n}\right)
$$

Proof. We have

$$
k(a)(\theta)=\psi(\theta) k(a)(\theta)+\chi(\theta) k(a)(\theta) .
$$

Then (3.9) follows from (3.4). Because of

$$
a(\xi)=V_{F}(\psi) a(\xi)+V_{F}(\chi) a(\xi)
$$

and the continuity of $V_{F}(\chi): S_{(\mathrm{cl})}^{\mu}\left(\mathbb{R}^{n}\right) \rightarrow S^{-\infty}\left(\mathbb{R}^{n}\right)$ which is a consequence of Lemma 1 we obtain the continuity of (3.8).

We have $\psi(\theta) k(a)(\theta) \in \mathscr{S}^{\prime}\left(\mathbb{R}^{q}\right)$ and

$$
\operatorname{supp}(\psi k(a)) \text { compact. }
$$

Thus $V_{F}(\psi) a(\xi)$ admits the interpretation of an operator $V_{F}(\psi): S_{(\mathrm{cl})}^{\mu}\left(\mathbb{R}^{n}\right) \rightarrow \mathscr{A}\left(\mathbb{C}_{\zeta}^{n}\right)$ for $\zeta:=$ $\xi+i \delta \in \mathbb{C}^{n}$, such that $\left.\boldsymbol{V}_{F}(\psi) a\right|_{\operatorname{Im} \zeta=0}=V_{F}(\psi) a(\xi)$. We shall see below that

$$
\boldsymbol{V}_{F}(\psi) a(\xi+i \delta) \in S_{(\mathrm{cl})}^{\mu}\left(\mathbb{R}_{\xi}^{n}\right) \quad \text { and } \quad V_{F}(\psi) a(\xi+i \delta)=a(\xi) \bmod S_{(\mathrm{cl})}^{\mu-1}\left(\mathbb{R}^{n}\right)
$$

for every fixed $\delta \in \mathbb{R}^{n}$, uniformly in compact sets in $\mathbb{R}_{\delta}^{n}$.

It is desirable to extend the definition of $V_{F}(\cdot)$ to arbitrary $\varphi \in C_{0}^{\infty}\left(\mathbb{R}^{n}\right)$, and even to $\varphi$ in the space

$$
C^{\infty}\left(\mathbb{R}^{n}\right)_{\mathrm{b}}:=\left\{\varphi \in C^{\infty}\left(\mathbb{R}^{n}\right): \sup _{\theta \in \mathbb{R}^{n}}\left|D_{\theta}^{\alpha} \varphi(\theta)\right|<\infty \text { for all } \alpha \in \mathbb{N}^{n}\right\}
$$


Clearly, for $\varphi \in C^{\infty}\left(\mathbb{R}^{n}\right)_{\mathrm{b}}$ in general we lose the existence of holomorphic extensions to the complex space $\mathbb{C}^{n}$.

Observe that $V_{F}(\varphi) a(\xi)=F_{\theta \rightarrow \xi}(\varphi(\theta) k(a)(\theta))(\xi)$, can be written as an oscillatory integral

$$
V_{F}(\varphi) a(\xi)=\int e^{-i \theta \xi} \varphi(\theta)\left\{\int e^{i \theta \xi^{\prime}} a\left(\xi^{\prime}\right) d \xi^{\prime}\right\} d \theta=\iint e^{-i \theta \tilde{\xi}} \varphi(\theta) a(\xi-\tilde{\xi}) d \theta d \tilde{\xi} .
$$

In order to unify the terminology we call $V_{F}(\varphi)$ a kernel cut-off operator also when $\varphi$ is more general than a cut-off function, and the same notation is used for $V_{F}(\varphi)$. This concerns again an extension of $V_{F}(\varphi) a(\xi)$ for $\varphi \in C_{0}^{\infty}\left(\mathbb{R}^{n}\right)$ into the complex space $\mathbb{C}^{n} \ni \zeta=\xi+i \delta$ which defines an operator $V_{F}(\varphi): S_{(\mathrm{cl})}^{\mu}\left(\mathbb{R}^{n}\right) \rightarrow \mathscr{A}\left(\mathbb{C}_{\zeta}^{n}\right)$ such that

$$
\left.V_{F}(\varphi) a\right|_{\operatorname{Im} \zeta=0}=V_{F}(\varphi) a(\xi) .
$$

In our applications we will employ several generalisations of kernel cut-off operators, in particular, to parameter-dependent symbols

$$
a(\xi, \lambda) \in S_{(\mathrm{cl})}^{\mu}\left(\mathbb{R}_{\xi, \lambda}^{n+l}\right),
$$

$l \in \mathbb{N}$. For $k(a)(\theta, \lambda):=\int e^{i \theta \xi} a(\xi, \lambda) d \xi$ we then have

$$
k(a)(\theta, \lambda) \in \mathscr{S}^{\prime}\left(\mathbb{R}_{\theta, \lambda}^{n+l}\right) \text {, and } \chi(\theta) k(a)(\theta, \lambda) \in \mathscr{S}\left(\mathbb{R}_{\theta, \lambda}^{n+l}\right),
$$

i.e., for an excision function $\chi(\theta)$

$$
V_{F}(\chi) a(\xi, \lambda)=F_{\theta \rightarrow \xi}(\chi k(a))(\xi, \lambda) \in \mathscr{S}\left(\mathbb{R}_{\xi, \lambda}^{n+l}\right)=S^{-\infty}\left(\mathbb{R}_{\xi, \lambda}^{n+l}\right) .
$$

Then $V_{F}(\psi):=1-V_{F}(\chi)$ for $\psi(\theta)=1-\chi(\theta)$ is a corresponding kernel cut-off operator in the parameter-dependent set-up, where

$$
V_{F}(\psi) a(\xi, \lambda)=a(\xi, \lambda) \bmod S^{-\infty}\left(\mathbb{R}^{n+l}\right) .
$$

More generally for $\varphi \in C^{\infty}\left(\mathbb{R}^{n}\right)_{\mathrm{b}}$ we form

$$
V_{F}(\varphi) a(\xi, \lambda)=\iint e^{-i \theta \tilde{\xi}} \varphi(\theta) a(\xi-\tilde{\xi}, \lambda) d \theta d \tilde{\xi} .
$$

For $\varphi \in C_{0}^{\infty}\left(\mathbb{R}^{n}\right)$ it follows that $V_{F}(\varphi) a(\xi, \lambda)$ extends to a function $V_{F}(\varphi) a(\zeta, \lambda)$ which is holomorphic in $\zeta \in \mathbb{C}^{n}$, and, as we shall show below,

$$
\boldsymbol{V}_{F}(\varphi) a(\xi+i \delta, \lambda) \in S_{(\mathrm{cl})}^{\mu}\left(\mathbb{R}_{\xi, \lambda}^{n+l}\right)
$$

for every $\delta \in \mathbb{R}^{n}$, uniformly in compact sets, and

$$
\boldsymbol{V}_{F}(\varphi) a(\xi+i \delta, \lambda)=\boldsymbol{V}_{F}(\varphi) a(\xi, \lambda) \bmod S_{(\mathrm{cl})}^{\mu-1}\left(\mathbb{R}^{n+l}\right)
$$

for every $\delta \in \mathbb{R}^{n}$. 
Remark 9. We have

$$
D_{\xi, \lambda}^{\alpha} V_{F}(\varphi) a(\xi, \lambda)=V_{F}(\varphi) D_{\xi, \lambda}^{\alpha} a(\xi, \lambda)
$$

for every $\alpha \in \mathbb{N}^{n+l}$.

In fact, using the first identity of (3.3) we obtain

$$
\begin{aligned}
D_{\xi}^{\alpha} V_{F}(\varphi) a(\xi, \lambda) & =\int e^{-i \xi \theta} \varphi(\theta)(-\theta)^{\alpha} k(a)(\theta, \lambda) d \theta \\
& =\int e^{-i \xi \theta} \varphi(\theta) k\left(D_{\xi}^{\alpha} a\right)(\theta, \lambda) d \theta=V_{F}(\varphi) D_{\xi}^{\alpha} a(\xi, \lambda) .
\end{aligned}
$$

Clearly the derivatives in $\lambda$ also commute with the kernel cut-off operator.

If $E$ is a Fréchet space with a countable semi-norm system $\left(p_{l}\right)_{l \in \mathbb{N}}$, we set

$$
C^{\infty}\left(\mathbb{R}^{n}, E\right)_{\mathrm{b}}:=\left\{\varphi \in C^{\infty}\left(\mathbb{R}^{n}, E\right): \sup _{\theta \in \mathbb{R}^{n}} p_{l}\left(D_{\theta}^{\alpha} \varphi(\theta)\right)<\infty \text { for all } \alpha \in \mathbb{N}^{n}, l \in \mathbb{N}\right\}
$$

Remark 10. We saw that the kernel cut-off operators $V_{F}(\varphi)$ act with respect to the covariables. This allows us to extend the constructions to symbols

$$
a(x, \xi, \lambda) \in S_{(\mathrm{cl})}^{\mu}\left(U \times \mathbb{R}_{\xi, \lambda}^{n+l}\right),
$$

depending on further variables $x \in U, U \subseteq \mathbb{R}^{m}$ open. The idea is simply to apply $V_{F}(\varphi)$ to $a\left(x_{0}, \xi, \lambda\right) \in S_{(\mathrm{cl})}^{\mu}\left(\mathbb{R}_{\xi, \lambda}^{n+l}\right)$ for every fixed $x_{0} \in U$. We also can take $U=\mathbb{R}^{m}$ and require the symbolic estimates uniformly in $x \in \mathbb{R}^{m}$. Since those symbol spaces are functions in $C^{\infty}\left(\mathbb{R}^{m}\right.$, $\left.S_{(\mathrm{cl})}^{\mu}\left(\mathbb{R}_{\xi, \lambda}^{n+l}\right)\right)_{\mathrm{b}}$ the essential information comes from the case with constant coefficients.

Let us now complete the information on kernel cut-off operators and give the outstanding proofs, starting with the oscillatory integral (3.17) for any $\varphi \in C^{\infty}\left(\mathbb{R}^{n}\right)_{\mathrm{b}}$. Our main applications concern the case $n=1$. This will be assumed from now on.

Theorem 3. The kernel cut-off operator $V_{F}:(\varphi, a) \rightarrow V_{F}(\varphi)$ a defines a bilinear continuous mapping

$$
V_{F}: C^{\infty}(\mathbb{R})_{\mathrm{b}} \times S_{(\mathrm{cl})}^{\mu}\left(\mathbb{R}_{\xi, \lambda}^{1+l}\right) \rightarrow S_{(\mathrm{cl})}^{\mu}\left(\mathbb{R}_{\xi, \lambda}^{1+l}\right)
$$

and $V_{F}(\varphi) a(\xi, \lambda)$ admits an asymptotic expansion

$$
V_{F}(\varphi) a(\xi, \lambda) \sim \sum_{k=0}^{\infty} \frac{(-1)^{k}}{k !} D_{\theta}^{k} \varphi(0) \partial_{\xi}^{k} a(\xi, \lambda) .
$$

Proof. The mapping $C^{\infty}\left(\mathbb{R}_{\theta}\right)_{\mathrm{b}} \times S_{(\mathrm{cl})}^{\mu}\left(\mathbb{R}_{\xi, \lambda}^{1+l}\right) \rightarrow C^{\infty}\left(\mathbb{R}_{\xi, \lambda}^{1+l}, S_{(\mathrm{cl})}^{\mu}\left(\mathbb{R}_{\theta} \times \mathbb{R}_{\tilde{\xi}}\right)_{\mathrm{b}}\right)$ for $S_{(\mathrm{cl})}^{\mu}\left(\mathbb{R}_{\theta} \times \mathbb{R}_{\tilde{\xi}}\right)_{\mathrm{b}}:=$ $C^{\infty}\left(\mathbb{R}_{\theta}, S_{(\mathrm{cl})}^{\mu}\left(\mathbb{R}_{\tilde{\xi}}\right)\right)_{\mathrm{b}}$, defined by $(\varphi, a) \rightarrow \varphi(\theta) a(\xi-\tilde{\xi}, \lambda)$, is bilinear continuous. In order to show the continuity of (3.19) it suffices to verify that $V_{F}(\varphi) a \in S_{(\mathrm{cl})}^{\mu}\left(\mathbb{R}_{\xi, \lambda}^{1+l}\right)$ and to apply the closed 
graph theorem. Since $D_{\xi, \lambda}^{\beta} V_{F}(\varphi) a(\xi, \lambda)=V_{F}(\varphi)\left(D_{\xi, \lambda}^{\beta} a(\xi, \lambda)\right)$ for any $\beta \in \mathbb{N}^{1+l}$, it suffices to show

$$
\left|V_{F}(\varphi) a(\xi, \lambda)\right| \leq c\langle\xi, \lambda\rangle^{\mu}
$$

for all $(\mu, \lambda) \in \mathbb{R}^{1+l}$, for a constant $c>0$. We regularise the oscillatory integral (3.17) as follows:

$$
V_{F}(\varphi) a(\xi, \lambda)=\iint e^{-i \theta \tilde{\xi}}\langle\theta\rangle^{-2}\left\{\left(1-\partial_{\theta}^{2}\right)^{N} \varphi(\theta)\right\} a_{N}(\xi, \tilde{\xi}, \lambda) d \theta d \tilde{\xi},
$$

where

$$
a_{N}(\xi, \tilde{\xi}, \lambda)=\left(1-\partial_{\tilde{\xi}}^{2}\right)\left\{\langle\tilde{\xi}\rangle^{-2 N} a(\xi-\tilde{\xi}, \lambda)\right\}
$$

for $N \in \mathbb{N}$ sufficiently large. The function (3.22) is a linear combination of terms $\left(\partial_{\tilde{\xi}}^{j}\langle\tilde{\xi}\rangle^{-2 N}\right)$ $\left(\partial_{\xi}^{k} a\right)(\xi-\tilde{\xi}, \lambda)$ for $0 \leq j, k \leq 2$. For the following conclusions we recall Peetre's inequality $\left\langle\xi^{\prime}+\right.$ $\left.\xi^{\prime \prime}\right\rangle^{s} \leq c^{|s|}\left\langle\xi^{\prime}\right\rangle^{|s|}\left\langle\xi^{\prime \prime}\right\rangle^{s}$ for all $\xi^{\prime}, \xi^{\prime \prime} \in \mathbb{R}, s \in \mathbb{R}$. We have $\langle\xi-\tilde{\xi}, \lambda\rangle^{\mu} \leq C\langle\tilde{\xi}\rangle^{|\mu|}\langle\xi, \lambda\rangle^{\mu}$, when we write $(\xi-\tilde{\xi}, \lambda)=(\xi, \lambda)-(\tilde{\xi}, 0)$. It follows that

$$
\begin{aligned}
& \left|\partial_{\tilde{\xi}}^{j}\langle\tilde{\xi}\rangle^{-2 N}\left(\partial_{\xi}^{k} a\right)(\xi-\tilde{\xi}, \lambda)\right| \leq\left|\partial_{\tilde{\xi}}^{j}\langle\tilde{\xi}\rangle^{-2 N}\right|\left|\left(\partial_{\xi}^{k} a\right)(\xi-\tilde{\xi}, \lambda)\right| \\
& \quad \leq c\langle\tilde{\xi}\rangle^{-2 N}\langle\xi-\tilde{\xi}, \lambda\rangle^{\mu} \leq c\langle\tilde{\xi}\rangle^{|\mu|-2 N}\langle\xi, \lambda\rangle^{\mu}
\end{aligned}
$$

for some $c>0$. This implies analogous estimates for the function (3.22). For $N$ so large that $\mu-2 N \leq 0$ we obtain the estimate (3.21).

In order to show (3.20) we employ the Taylor expansion

$$
\varphi(\theta)=\sum_{k=0}^{N} \frac{1}{k !}\left(\partial_{\theta}^{k} \varphi\right)(0) \theta^{k}+\theta^{N+1} \varphi_{N+1}(\theta),
$$

for $\varphi_{N+1}(\theta)=\frac{1}{N !} \int_{0}^{1}(1-t)^{N}\left(\partial_{\theta}^{N+1} \varphi\right)(t \theta) d t$. The function $\varphi_{N+1}(\theta)$ belongs to $C^{\infty}(\mathbb{R})_{\mathrm{b}}$. Integration by parts in (3.17) gives us

$$
\begin{aligned}
V_{F}(\varphi) a(\xi, \lambda)= & \sum_{k=0}^{N} \frac{1}{k !}\left(\partial_{\theta}^{k} \varphi\right)(0) \iint e^{-i \theta \tilde{\xi}} \theta^{k} a(\xi-\tilde{\xi}, \lambda) d \theta d \tilde{\xi} \\
& +\iint e^{-i \theta \tilde{\xi}} \theta^{N+1} \varphi_{N+1}(\theta) a(\xi-\tilde{\xi}, \lambda) d \theta d \tilde{\xi} \\
= & \sum_{k=0}^{N} \frac{(-1)^{k}}{k !}\left(D_{\theta}^{k} \varphi\right)(0)\left(\partial_{\xi}^{k} a\right)(\xi, \lambda)+(-1)^{N+1} V_{F}\left(\varphi_{N+1}\right) \partial_{\xi}^{N+1} a(\xi, \lambda) .
\end{aligned}
$$

In the latter equation we employed the identity

$$
\left(\partial_{\xi}^{k} a\right)(\xi, \lambda)=\iint e^{-i \theta \tilde{\xi}}\left(\partial_{\xi}^{k} a\right)(\xi-\tilde{\xi}, \lambda) d \theta d \tilde{\xi}
$$

and the expression (3.17), applied to $\varphi_{N+1}$. From the first part of the proof we know that $V_{F}\left(\varphi_{N+1}\right)\left(\partial_{\xi}^{N+1} a\right)(\xi, \lambda)$ is a symbol in $S^{\mu-(N+1)}\left(\mathbb{R}^{1+l}\right)$. This completes the proof of (3.20). 
Corollary 1. Let $\psi(\theta) \in C_{0}^{\infty}(\mathbb{R})$ be a cut-off function on $\mathbb{R}$ (i.e., $\psi \equiv 1$ in a neighbourhood of 0$)$, and $\operatorname{set} \psi_{\varepsilon}(\theta)=\psi(\varepsilon \theta)$ for $0<\varepsilon \leq 1$. Then for every $a(\xi, \lambda) \in S_{(\mathrm{cl})}^{\mu}\left(\mathbb{R}^{1+l}\right)$ we have

$$
\lim _{\varepsilon \rightarrow 0} V_{F}\left(\psi_{\varepsilon}\right) a(\xi, \lambda)=a(\xi, \lambda)
$$

in the topology of $S_{(\mathrm{cl})}^{\mu}\left(\mathbb{R}^{1+l}\right)$.

In fact, we have $\lim _{\varepsilon \rightarrow 0} \psi_{\varepsilon}=1$ in the topology of $C^{\infty}(\mathbb{R})_{\mathrm{b}}$; then (3.23) is a consequence of the continuity of (3.19) for a fixed symbol on the left hand side of (3.19).

Corollary 2. Let $\varphi \in C^{\infty}(\mathbb{R})_{\mathrm{b}}$ have the property $\partial_{\theta}^{k} \varphi(0)=0$ for all $0 \leq k \leq N$. Then $V_{F}(\varphi)$ defines a continuous mapping

$$
V_{F}(\varphi): S_{(\mathrm{cl})}^{\mu}\left(\mathbb{R}^{1+l}\right) \rightarrow S_{(\mathrm{cl})}^{\mu-(N+1)}\left(\mathbb{R}^{1+l}\right)
$$

This is a consequence of the asymptotic expansion (3.20), combined with the continuity of (3.19) for fixed $\varphi$ on the left hand side of (3.19).

Corollary 3. Let $\chi(\theta)$ be an excision function. Then $V_{F}(\chi)$ defines a continuous operator

$$
V_{F}(\chi): S_{(\mathrm{cl})}^{\mu}\left(\mathbb{R}^{1+l}\right) \rightarrow S^{-\infty}\left(\mathbb{R}^{1+l}\right)=\mathscr{S}\left(\mathbb{R}_{\xi, \lambda}^{1+l}\right)
$$

In particular,

$$
\chi(\theta) k(a)(\theta, \lambda) \in \mathscr{S}\left(\mathbb{R}_{\theta, \lambda}^{1+l}\right)
$$

for every $a(\xi, \lambda) \in S_{(\mathrm{cl})}^{\mu}\left(\mathbb{R}^{1+l}\right)$.

Remark 11. By virtue of the way to regularise the oscillatory integral (3.17) it is also possible to generalise $V_{F}(\varphi)$ to functions $\varphi \in C^{\infty}(\mathbb{R})$ satisfying the estimates

$$
\left|D_{\theta}^{k} \varphi(\theta)\right| \leq c_{k}\langle\theta\rangle^{v} \text { for all } k \in \mathbb{N},
$$

for constants $c_{k}>0$; the number $v \in \mathbb{R}$ is arbitrary and fixed.

Definition 3. Let $S_{(\mathrm{cl})}^{\mu}\left(C \times \mathbb{R}^{l}\right)$ denote the space of all $h(\zeta, \lambda) \in \mathscr{A}\left(\mathbb{C}, S_{(\mathrm{cl})}^{\mu}\left(\mathbb{R}_{\lambda}^{l}\right)\right)$ such that

$$
\left.h\right|_{I_{\delta} \times \mathbb{R}^{l}} \in S_{(\mathrm{cl})}^{\mu}\left(I_{\delta} \times \mathbb{R}^{l}\right)
$$

for every $\delta \in \mathbb{R}$, uniformly in compact $\delta$-intervals; $I_{\delta}=\{\zeta \in \mathbb{C}: \operatorname{Im} \zeta=\delta\}$.

The spaces $S_{(\mathrm{cl})}^{\mu}\left(\boldsymbol{C} \times \mathbb{R}^{l}\right)$ are Fréchet in a natural way. For any $K \Subset \mathbb{R}$ we set $C_{\mathbf{0}}^{\infty}(K):=\{u \in$ $C^{\infty}(\mathbb{R})$ : supp $\left.u \subseteq K\right\}$ which is a Fréchet space.

Theorem 4. For every $K \Subset \mathbb{R}$ the kernel cut-off operator $V_{F}:(\varphi, a) \rightarrow V_{F}(\varphi)$ a induces a bilinear continuous mapping

$$
\boldsymbol{V}_{F}: C_{\mathbf{0}}^{\infty}(K) \times S_{(\mathrm{cl})}^{\mu}\left(\mathbb{R}_{\xi, \lambda}^{1+l}\right) \rightarrow S_{(\mathrm{cl})}^{\mu}\left(\boldsymbol{C} \times \mathbb{R}^{l}\right)
$$


Proof. We have

$$
k(a)(\theta, \lambda)=\int e^{i \theta \xi^{\prime}} a\left(\xi^{\prime}, \lambda\right) d \xi^{\prime} \in \mathscr{S}^{\prime}\left(\mathbb{R}_{\theta, \lambda}^{1+l}\right),
$$

and $\varphi(\theta) k(a)(\theta, \lambda)$ has compact support with respect to $\theta$ for $\varphi \in C_{\mathbf{0}}^{\infty}(K), K \Subset \mathbb{R}$. Thus $V_{F}(\varphi) a(\xi, \lambda)=\int e^{-i \theta \eta} \varphi(\theta) k(a)(\theta, \lambda) d \theta$ extends to a function

$$
V_{F}(\varphi) a(\xi+i \delta, \lambda)=\int e^{-i \theta \xi} e^{\theta \delta} \varphi(\theta) k(a)(\theta, \lambda) d \theta=V_{F}\left(\varphi_{\delta}\right) a(\xi, \lambda),
$$

$\varphi_{\delta}(\theta):=e^{\theta \delta} \varphi(\theta) \in C_{\mathbf{0}}^{\infty}(K)$, which is holomorphic in $\zeta=\xi+i \delta \in \mathbb{C}$. Theorem 3 yields $V_{F}(\varphi) a(\xi+$ $i \delta, \lambda) \in S_{(\mathrm{cl})}^{\mu}\left(\mathbb{R}_{\xi, \lambda}^{1+l}\right)$ for every $\delta$. Since $\delta \rightarrow \varphi_{\delta}$ represents a continuous mapping $\mathbb{R} \rightarrow C_{\mathbf{0}}^{\infty}(K)$ the continuity of

$$
V_{F}: C_{\mathbf{0}}^{\infty}(K) \times S_{(\mathrm{cl})}^{\mu}\left(\mathbb{R}_{\xi, \lambda}^{1+l}\right) \rightarrow S_{(\mathrm{cl})}^{\mu}\left(\mathbb{R}_{\xi, \lambda}^{1+l}\right)
$$

implies that (3.24) is uniform in compact $\delta$-intervals. The closed graph theorem gives us also the continuity of (3.25) with respect to the Fréchet topology of the space $S_{(\mathrm{cl})}^{\mu}\left(\boldsymbol{C} \times \mathbb{R}^{l}\right)$.

Corollary 4. Let $\psi \in C_{0}^{\infty}(\mathbb{R})$ be a cut-off function, and $a(\xi, \lambda) \in S_{(\mathrm{cl})}^{\mu}\left(\mathbb{R} \times \mathbb{R}^{l}\right)$. Then we have

$$
h(\zeta, \lambda):=\boldsymbol{V}_{F}(\psi) a(\zeta, \lambda) \in S_{(\mathrm{cl})}^{\mu}\left(\boldsymbol{C} \times \mathbb{R}^{l}\right)
$$

and

$$
\left.h(\xi+i \delta, \lambda)\right|_{\delta=0}=a(\xi, \lambda) \bmod S^{-\infty}\left(\mathbb{R}^{1+l}\right) .
$$

In fact, the relation (3.27) is a consequence of Theorem 4, and (3.28) follows from

$$
a(\xi, \lambda)-h(\xi, \lambda)=V_{F}(1-\psi) a(\xi, \lambda)
$$

together with Corollary 2.

Corollary 5. Let $\varphi \in C_{0}^{\infty}(\mathbb{R})$ and define

$$
h_{\delta}(\xi, \lambda):=V_{F}(\varphi) a(\xi+i \delta, \lambda)
$$

for any fixed $\delta \in \mathbb{R}$. Then $a \rightarrow h_{\delta}$ defines a continuous map

$$
S_{(\mathrm{cl})}^{\mu}\left(\mathbb{R}^{1+l}\right) \rightarrow S_{(\mathrm{cl})}^{\mu}\left(\mathbb{R}^{1+l}\right)
$$

and we have

$$
h_{0}(\xi, \lambda)=h_{\delta}(\xi, \lambda) \bmod S_{(\mathrm{cl})}^{\mu-1}\left(\mathbb{R}^{1+l}\right)
$$

In fact, the continuity (3.29) is a direct consequence of the continuity of (3.25). Moreover, the relation (3.30) is a consequence of the asymptotic expansion (3.20) applied to the function $e^{\theta \delta} \varphi(\theta)$, cf. the formula (3.26). 
Lemma 2. Let $h(\zeta, \lambda) \in S_{(\mathrm{cl})}^{\mu}\left(\boldsymbol{C} \times \mathbb{R}^{l} ; H, \widetilde{H}\right)$, and set $h_{\delta}(\xi, \lambda):=h(\xi+i \delta, \lambda)$. Then the mapping,

$$
\mathbb{R} \rightarrow S_{(\mathrm{cl})}^{\mu}\left(\mathbb{R}_{\xi, \lambda}^{1+l}\right), \delta \rightarrow h_{\delta},
$$

is continuous.

Proof. By virtue of the holomorphy of $h$ in $\zeta$ we have

$$
\left(h_{\delta}-h_{\delta_{0}}\right)(\xi, \lambda)=\left(\delta-\delta_{0}\right) \int_{0}^{1} D_{\xi} h_{\delta_{0}+\theta\left(\delta-\delta_{0}\right)}(\xi, \lambda) d \theta .
$$

Let $\pi$ be one of the semi-norms of the Fréchet topology of $S^{\mu}\left(\mathbb{R}^{1+l}\right)$. Then for $\left|\delta-\delta_{0}\right| \leq \varepsilon$ for any $\varepsilon>0$ we have

$$
\pi\left(h_{\delta}-h_{\delta_{0}}\right) \leq\left|\delta-\delta_{0}\right| \sup \left\{\pi\left(D_{\xi} h_{\delta^{\prime}}\right):\left|\delta^{\prime}-\delta_{0}\right| \leq \varepsilon\right\} .
$$

By assumption we have $h_{\delta^{\prime}}(\xi, \lambda) \in S^{\mu}\left(\mathbb{R}^{1+l}\right)$ uniformly in compact $\delta^{\prime}$-intervals. The same is true of $D_{\xi} h_{\delta^{\prime}}$. This shows $\pi\left(h_{\delta}-h_{\delta_{0}}\right) \rightarrow 0$ as $\delta \rightarrow \delta_{0}$, and we obtain the continuity of $\mathbb{R} \rightarrow S^{\mu}\left(\mathbb{R}^{1+l}\right)$, since $\pi$ is arbitrary. In addition, for classical symbols we have to show the continuous in $\delta$ dependence of the homogeneous components $\left(h_{\delta}\right)_{(\mu-j)}(\xi, \lambda) \in S^{(\mu-j)}\left(\mathbb{R}^{1+l} \backslash\{0\}\right)$ and of the remainders

$$
h_{\delta}(\xi, \lambda)-\sum_{j=0}^{N} \chi(\xi, \lambda) h_{\delta,(\mu-j)}(\xi, \lambda) \in S^{\mu-(N+1)}\left(\mathbb{R}^{1+l}\right)
$$

for some excision function $\chi$. Taylor expansion of $h$ in $\delta$ at $\delta=0$ gives us

$$
h_{\delta}(\xi, \lambda)=\sum_{j=0}^{N} \frac{1}{j !} D_{\xi}^{j} h_{0}(\xi, \lambda) \delta^{j}+\delta^{N+1} \int_{0}^{1} \frac{(1-\theta)^{N}}{N !}\left(D_{\xi}^{N+1} h_{\theta \delta}\right)(\xi, \lambda) d \xi .
$$

The first part of the proof shows that the remainder $r_{\delta}$ is continuous in $\delta$ with values in $S^{\mu-(N+1)}\left(\mathbb{R}^{1+l}\right)$. Moreover, the homogeneous components $h_{\delta,(\mu-j)}$ in $(\xi, \lambda) \neq 0, j=0, \ldots, N$, coincide with the respective homogeneous components of the sum on the right hand side of (3.31). Those are polynomials in $\delta$ with coefficients in $S^{(\mu-j)}\left(\mathbb{R}^{1+l} \backslash\{0\}\right)$ and hence continuous in $\delta$.

Choose an excision function $\chi(\xi, \lambda)$, write $\chi h_{\delta,(\mu-k)}=\chi \sum_{j=0}^{N} \frac{1}{j !}\left(D_{\xi}^{j} h_{0}\right)_{(\mu-k)} \delta^{j}$ for $0 \leq k \leq$ $N$, and consider $\chi h_{\delta}=\chi \sum_{j=0}^{N} \frac{1}{j !}\left(D_{\xi}^{j} h_{0}\right) \delta^{j}+\chi r_{\delta}$. Then

$$
h_{\delta}=\chi h_{\delta}+(1-\chi) h_{\delta}=(1-\chi) h_{\delta}+\chi r_{\delta}+\chi \sum_{j=0}^{N} \frac{1}{j !}\left(D_{\xi}^{j} h_{0}\right) \delta^{j} .
$$

Subtracting from (3.31) the sum of the terms $\chi h_{\delta,(\mu-k)}$ over $k=0, \ldots, N$, yields

$$
h_{\delta}-\chi \sum_{k=0}^{N} h_{\delta,(\mu-k)}=\left((1-\chi) h_{\delta}+\chi r_{\delta}\right)+\chi \sum_{j=0}^{N} \frac{1}{j !}\left(D_{\xi}^{j} h_{0}-\sum_{k=0}^{N}\left(D_{\xi}^{j} h_{0}\right)_{(\mu-k)}\right) \delta^{j} .
$$

The first term on the right is continuous in $\delta$ with values in $S^{\mu-(N+1)}\left(\mathbb{R}^{1+l}\right)$. The second term is a polynomial in $\delta$ with coefficients in $S^{\mu-(N+1)}\left(\mathbb{R}^{1+l}\right)$ and as such also continuous in $\delta$. 
Proposition 2. For every $h(\zeta, \lambda) \in S_{(\mathrm{cl})}^{\mu}\left(C \times \mathbb{R}^{l}\right)$ we have

$$
\left(\partial_{\zeta} h\right)(\zeta, \lambda) \in S_{(\mathrm{cl})}^{\mu-1}\left(C \times \mathbb{R}^{l}\right)
$$

(here $\partial_{\zeta}$ is the differentiation of $h$ as a holomorphic function with values in $S_{(\mathrm{cl})}^{\mu}\left(\mathbb{R}^{l}\right)$ ).

Proof. Let us set $h_{\delta}(\eta, \lambda):=h(\xi+i \delta, \lambda)$. By Lemma 2 the mapping $\mathbb{R}^{2} \rightarrow S_{(\mathrm{cl})}^{\mu-1}\left(\mathbb{R}_{\xi, \lambda}^{1+l}\right),(\xi, \delta)$ $\rightarrow\left(\partial_{\xi} h_{\delta}\right)(\xi, \lambda)$, is continuous, and we have $\left(\partial_{\xi} h_{\delta}\right)(\xi, \lambda)=\left(\partial_{\zeta} h\right)(\eta+i \delta, \lambda)$. Thus $\zeta \rightarrow \partial_{\zeta} h \in$ $C\left(\mathbb{C}_{\zeta}, S_{(\mathrm{cl})}^{\mu-1}\left(\mathbb{R}_{\lambda}^{l}\right)\right) \cap \mathscr{A}\left(\mathbb{C}_{\zeta}, S_{(\mathrm{cl})}^{\mu}\left(\mathbb{R}_{\lambda}^{l}\right)\right)$. Then Cauchy's integral formula yields $\partial_{\zeta} h(\zeta, \lambda) \in$ $\mathscr{A}\left(\mathbb{C}, S_{(\mathrm{cl})}^{\mu-1}\left(\mathbb{R}_{\lambda}^{l}\right)\right)$. Since

$$
\left(\partial_{\zeta} h\right)_{\delta}=\partial_{\xi} h_{\delta} \in S_{(\mathrm{cl})}^{\mu-1}\left(\mathbb{R}_{\xi, \lambda}^{1+l}\right)
$$

uniformly in compact $\delta$-intervals, it follows that $\partial_{\zeta} h(\zeta, \lambda) \in S_{(\mathrm{cl})}^{\mu-1}\left(C \times \mathbb{R}^{l}\right)$.

Theorem 5. Let $h(\zeta, \lambda) \in S_{(\mathrm{cl})}^{\mu}\left(C \times \mathbb{R}^{l}\right)$ and fix $\delta \in \mathbb{R}$; then

(i) $h_{\delta} \in S_{(\mathrm{cl})}^{\mu-\varepsilon}\left(\mathbb{R}_{\xi, \lambda}^{1+l}\right)$ for some $\varepsilon \geq 0\left(\varepsilon \in \mathbb{N}\right.$ in the classical case) implies $h \in S_{(\mathrm{cl})}^{\mu-\varepsilon}\left(C \times \mathbb{R}^{l}\right)$;

(ii) $h_{\delta} \in S_{\mathrm{cl}}^{\mu}\left(\mathbb{R}_{\xi, \lambda}^{1+l}\right)$ implies $h \in S_{\mathrm{cl}}^{\mu}\left(C \times \mathbb{R}^{l}\right)$.

Proof. Without loss of generality we may assume $\delta=0$. Let us apply the Taylor expansion at $\delta=0$ :

$$
h(\xi+i \delta, \lambda)=\sum_{j=0}^{N} \frac{1}{j !}\left(\partial_{\xi}^{j} h_{0}\right)(\xi, \lambda) \delta^{j}+\delta^{N+1} \int_{0}^{1} \frac{(1-\theta)^{N}}{N !}\left(\partial_{\zeta}^{N+1} h\right)(\xi+i \theta \delta, \lambda) d \theta
$$

By virtue of Lemma 2 and Proposition 2 the remainder is continuous in $\delta$ with values in $S_{(\mathrm{cl})}^{\mu-(N+1)}\left(\mathbb{R}_{\xi, \lambda}^{1+l}\right)$. In the case (i) it follows that $h \in C\left(\mathbb{C}, S_{(\mathrm{cl})}^{\mu-\varepsilon}\left(\mathbb{R}_{\lambda}^{l}\right)\right)$ and, in the case (ii), $h \in$ $C\left(\mathbb{C}, S_{\mathrm{cl}}^{\mu}\left(\mathbb{R}_{\lambda}^{l}\right)\right.$. Then Cauchy's integral formula gives us

$$
h \in \mathscr{A}\left(\mathbb{C}, S_{(\mathrm{cl})}^{\mu-\varepsilon}\left(\mathbb{R}^{l}\right)\right) \text { and } h \in \mathscr{A}\left(\mathbb{C}, S_{\mathrm{cl}}^{\mu}\left(\mathbb{R}^{l}\right)\right)
$$

respectively. In addition we obtain $h_{\delta} \in S_{(\mathrm{cl})}^{\mu-\varepsilon}\left(\mathbb{R}_{\xi, \lambda}^{1+l}\right)$ and $h_{\delta} \in S_{\mathrm{cl}}^{\mu}\left(\mathbb{R}_{\xi, \lambda}^{1+l}\right)$, uniformly in compact $\delta$-intervals. This shows the assertion.

\subsection{Kernel cut-off based on the Mellin transform}

We now turn to the Mellin version of kernel cut-off operators. By using the elementary relationship between Fourier and Mellin transform the Mellin kernel cut-off can be reduced to the one based on the one-dimensional Fourier transform. However, because of some specific modifications we prefer to formulate some basics once again in direct form.

Let us start with a scalar symbol $f(z) \in S_{(\mathrm{cl})}^{\mu}\left(\Gamma_{0}\right)$. Concerning notation such as (2.33) we refer to the material around the Mellin transform in Subsection 2.2. For convenience we first 
focus on the weight $\gamma=1 / 2$. Later on we draw consclusions for arbitrary weights. Recall that the Mellin operator $\operatorname{Op}_{M}^{1 / 2}(f) u(r)$, cf. (2.36), is interpreted as a Mellin oscillatory integral, or

$$
\mathrm{Op}_{M}^{1 / 2}(f) u(r)=\int_{0}^{\infty}\left\{\int_{-\infty}^{\infty}\left(r / r^{\prime}\right)^{-i \rho} f(i \rho) d \rho\right\} u\left(r^{\prime}\right) d r^{\prime} / r^{\prime}
$$

Then

$$
m(f)(s):=\int_{-\infty}^{\infty} s^{-i \rho} f(i \rho) d \rho=M_{1 / 2}^{-1} f(s),
$$

cf. (2.35), is a distribution on $\mathbb{R}_{+} \ni s$. Interpreting $\mathscr{T}\left(\mathbb{R}_{+}\right):=M_{1 / 2}^{-1} \mathscr{S}\left(\Gamma_{0}\right)$ as a Mellin analogue of the Schwartz space, we have $m(f)(s) \in \mathscr{T}^{\prime}\left(\mathbb{R}_{+}\right)$with $\mathscr{T}^{\prime}\left(\mathbb{R}_{+}\right)$being the dual of $\mathscr{T}\left(\mathbb{R}_{+}\right)$, the Mellin analogue of the space of temperate distributions. Note that

$$
(\log s)^{j} m(f)(s)=m\left(D_{\rho}^{j} f\right)(s),\left(-s \frac{\partial}{\partial s}\right)^{j} m(f)(s)=m\left((i \rho)^{j} f\right)(s), j \in \mathbb{N} .
$$

Setting $s:=e^{-\theta}, \theta \in \mathbb{R}$, it follows that

$$
m(f)\left(e^{-\theta}\right)=\int_{-\infty}^{\infty} e^{i \rho \theta} f(i \rho) d \rho=k(f(i \cdot))(\theta),
$$

cf. notation (3.2). In this section a cut-off function will be any $\psi(s) \in C_{0}^{\infty}\left(\mathbb{R}_{+, s}\right)$ that is equal to 1 in a neighbourhood of $s=1$. Moreover, an excision function will be any $\chi(s) \in C^{\infty}\left(\mathbb{R}_{+, s}\right)$ such that $1-\chi(s)$ is a cut-off function. From Lemma 1 we know that $\chi\left(e^{-\theta}\right) m(f)\left(e^{-\theta}\right) \in \mathscr{S}\left(\mathbb{R}_{\theta}\right)$ for every excision function $\chi(s)$. In particular, we obtain

$$
\operatorname{sing} \operatorname{supp} m(f) \subseteq\{1\}
$$

Set

$$
C^{\infty}\left(\mathbb{R}_{+}\right)_{\mathrm{B}}=\left\{\varphi \in C^{\infty}\left(\mathbb{R}_{+}\right): \sup _{s \in \mathbb{R}_{+}}\left|\left(s \partial_{s}\right)^{j} \varphi(s)\right|<\infty \text { for all } j \in \mathbb{N}\right\} .
$$

Observe that the function pull back under the diffeomorphism $\mathbb{R} \rightarrow \mathbb{R}_{+}, \theta \rightarrow e^{-\theta}=: s$ induces an isomorphism

$$
C^{\infty}\left(\mathbb{R}_{+}\right)_{\mathrm{B}} \rightarrow C^{\infty}(\mathbb{R})_{\mathrm{b}},
$$

cf. (3.13). For any $\varphi \in C^{\infty}\left(\mathbb{R}_{+}\right)_{\mathrm{B}}$ we set

$$
V_{M}(\varphi)(f):=M_{1 / 2, s \rightarrow z}(\varphi m(f))(s),
$$

called a Mellin kernel cut-off operator.

Proposition 3. The operator $V_{M}(\psi)$ for a cut-off function $\psi$ induces a continuous map

$$
V_{M}(\psi): S_{(\mathrm{cl})}^{\mu}\left(\Gamma_{0}\right) \rightarrow S_{(\mathrm{cl})}^{\mu}\left(\Gamma_{0}\right)
$$

where

$$
V_{M}(\psi) f=f \bmod S^{-\infty}\left(\Gamma_{0}\right)
$$


Proof. We have $m(f)(s)=\psi(s) m(f)(s)+\chi(s) m(f)(s)$ for $\chi(s)=1-\psi(s)$. Then (3.41) follows from

$$
\chi(s) m(f)(s) \in \mathscr{T}\left(\mathbb{R}_{+}\right) .
$$

Because of $f=V_{M}(\psi) f+V_{M}(\chi) f$ and the continuity of $V_{M}(\chi): S_{(\mathrm{cl})}^{\mu}\left(\Gamma_{0}\right) \rightarrow S^{-\infty}\left(\Gamma_{0}\right)$ which is a consequence of (3.42) we obtain the continuity of (3.40).

We have $\psi(s) m(f)(s) \in \mathscr{T}^{\prime}\left(\mathbb{R}_{+}\right)$and

$$
\operatorname{supp}(\psi m(f)) \text { compact. }
$$

Thus $V_{M}(\psi) f(i \rho)$ admits the interpretation of an operator $V_{M}(\psi): S_{(\mathrm{cl})}^{\mu}\left(\Gamma_{0}\right) \rightarrow \mathscr{A}\left(\mathbb{C}_{z}\right)$ for $z:=$ $\beta+i \rho \in \mathbb{C}$, such that $\left.\boldsymbol{V}_{M}(\psi) f\right|_{\operatorname{Re} z=0}=V_{M}(\psi) f(i \rho)$. We shall see below that

$$
\boldsymbol{V}_{M}(\psi) f(\beta+i \rho) \in S_{(\mathrm{cl})}^{\mu}\left(\Gamma_{\beta}\right) \quad \text { and } \quad V_{M}(\psi) f(\beta+i \rho)=f(i \rho) \bmod S_{(\mathrm{cl})}^{\mu-1}\left(\Gamma_{0}\right)
$$

for every fixed $\beta \in \mathbb{R}$, uniformly in compact sets in $\mathbb{R}_{\beta}$.

The expression $V_{M}(\varphi) f(i \rho)$ will be interpreted as a Mellin oscillatory integral

$$
V_{M}(\varphi) f(i \rho)=M_{1 / 2, s \rightarrow \rho} \varphi(s) \int s^{-i \rho^{\prime}} f\left(i \rho^{\prime}\right) d \rho^{\prime}=\iint s^{i \tilde{\rho}} \varphi(s) f(i(\rho-\tilde{\rho})) d s / s d \tilde{\rho} .
$$

For our applications below we admit parameter-dependent amplitude functions with parameters $\lambda \in \mathbb{R}^{l}$. First let

$$
f(i \rho, \lambda) \in S^{\mu}\left(\Gamma_{0} \times \mathbb{R}^{l}\right)
$$

Theorem 6. The kernel cut-off operator $V_{M}:(\varphi, f) \rightarrow V_{M}(\varphi) f$ defines a bilinear continuous mapping

$$
V_{M}: C^{\infty}\left(\mathbb{R}_{+}\right)_{\mathrm{B}} \times S_{(\mathrm{cl})}^{\mu}\left(\Gamma_{0} \times \mathbb{R}^{l}\right) \rightarrow S_{(\mathrm{cl})}^{\mu}\left(\Gamma_{0} \times \mathbb{R}^{l}\right),
$$

and $V_{M}(\varphi) f(i \rho, \lambda)$ admits an asymptotic expansion

$$
V_{M}(\varphi) f(i \rho, \lambda) \sim \sum_{k=0}^{\infty} \frac{1}{k !}\left(\left(s \partial_{s}\right)^{k} \varphi\right)(1) \partial_{\rho}^{k} f(i \rho, \lambda) .
$$

Proof. We reduce the proof to that of Theorem 3. The expression (3.45) will be applied to $f(i \rho, \lambda)=: a(\rho, \lambda)$, and we substitute $s=e^{-\theta}$. Then we obtain

$$
V_{M}(\varphi) f(i \rho, \lambda)=\iint e^{-i \theta \tilde{\rho}} \varphi\left(e^{-\theta}\right) a(\rho-\tilde{\rho}, \lambda) d \theta d \tilde{\rho} .
$$

By virtue of the isomorphism (3.38) we can apply Theorem 3 which gives us the bilinear continuous mapping (3.47). For the asymptotic expansion (3.48) it suffices to apply (3.20), using the relations $\partial_{\theta}^{k} \varphi\left(e^{-\theta}\right)=\left(-s \partial_{s}\right)^{k} \varphi(s)$, and $\left.\varphi\left(e^{-\theta}\right)\right|_{\theta=0}=\varphi(1)$. 
Let us generalise the kernel cut-off operator $V_{M}$ to the weighted Mellin transform $M_{\gamma}$, cf. the formula (2.34), applied to amplitude functions $f(1 / 2-\gamma+i \rho, \lambda) \in S_{(\mathrm{cl})}^{\mu}\left(\Gamma_{\frac{1}{2}-\gamma} \times \mathbb{R}^{l}\right)$. Instead of (3.45) we form

$$
\begin{aligned}
V_{M}(\varphi) f(1 / 2-\gamma+i \rho, \lambda) & =M_{\gamma, s \rightarrow \rho} \varphi(s) \int s^{-\left(1 / 2-\gamma+i \rho^{\prime}\right)} f\left(1 / 2-\gamma+i \rho^{\prime}, \lambda\right) d \rho^{\prime} \\
& =\iint s^{1 / 2-\gamma+i \rho} \varphi(s) s^{-\left(1 / 2-\gamma+i \rho^{\prime}\right)} f\left(1 / 2-\gamma+i \rho^{\prime}, \lambda\right) d s / s d \rho^{\prime} \\
& =\iint s^{i \tilde{\rho}} \varphi(s) f(1 / 2-\gamma+i(\rho-\tilde{\rho}), \lambda) d s / s d \tilde{\rho} .
\end{aligned}
$$

Corollary 6. Theorem 6 has a straightforward generalisation to the weighted Mellin transform, cf. (2.34), and instead of (3.47) we obtain a bilinear continuous mapping

$$
V_{M}: C^{\infty}(\mathbb{R})_{\mathrm{B}} \times S_{(\mathrm{cl})}^{\mu}\left(\Gamma_{1 / 2-\gamma} \times \mathbb{R}^{l}\right) \rightarrow S_{(\mathrm{cl})}^{\mu}\left(\Gamma_{1 / 2-\gamma} \times \mathbb{R}^{l}\right),
$$

and $V_{M}(\varphi) f(1 / 2-\gamma+i \rho, \lambda)$ admits an asymptotic expansion

$$
V_{M}(\varphi) f(1 / 2-\gamma+i \rho, \lambda) \sim \sum_{k=0}^{\infty} \frac{1}{k !}\left(\left(s \partial_{s}\right)^{k} \varphi\right)(1) \partial_{\rho}^{k} f(1 / 2-\gamma+i \rho, \lambda) .
$$

Corollary 7. Let $\psi(s) \in C_{0}^{\infty}\left(\mathbb{R}_{+}\right)$be a cut-off function on $\mathbb{R}_{+}$, and $\operatorname{set} \psi_{\varepsilon}(s):=\psi\left(s^{\varepsilon}\right)$ for $0<\varepsilon \leq 1$. Then for every $f(1 / 2-\gamma+i \rho, \lambda) \in S_{(\mathrm{cl})}^{\mu}\left(\Gamma_{1 / 2-\gamma} \times \mathbb{R}^{l}\right)$ we have

$$
\lim _{\varepsilon \rightarrow 0} V_{M}\left(\psi_{\varepsilon}\right) f(1 / 2-\gamma+i \rho, \lambda)=f(1 / 2-\gamma+i \rho, \lambda)
$$

in the topology of $S_{(\mathrm{cl})}^{\mu}\left(\Gamma_{1 / 2-\gamma} \times \mathbb{R}^{l}\right)$.

In fact, we have $\lim _{\varepsilon \rightarrow 0} \psi_{\varepsilon}=1$ in the topology of $C^{\infty}\left(\mathbb{R}_{+}\right)_{\mathrm{B}}$; then (3.53) is a consequence of the continuity of (3.51) for a fixed symbol on the left hand side of (3.51).

Corollary 8. Let $\varphi \in C^{\infty}\left(\mathbb{R}_{+}\right)_{\mathrm{B}}$ have the property $\left(s \partial_{s}\right)^{k} \varphi(1)=0$ for all $0 \leq k \leq N$. Then $V_{M}(\varphi)$ defines a continuous mapping

$$
V_{M}(\varphi): S_{(\mathrm{cl})}^{\mu}\left(\Gamma_{1 / 2-\gamma} \times \mathbb{R}^{l}\right) \rightarrow S_{(\mathrm{cl})}^{\mu-(N+1)}\left(\Gamma_{1 / 2-\gamma} \times \mathbb{R}^{l}\right) .
$$

This is a consequence of the asymptotic expansion (3.52), combined with the continuity of (3.51).

Corollary 9. Let $\chi(s) \in C^{\infty}\left(\mathbb{R}_{+}\right)_{\mathrm{B}}$ vanish of infinite order at $s=1$. Then $V_{M}(\chi)$ defines a continuous mapping

$$
V_{M}(\chi): S_{(\mathrm{cl})}^{\mu}\left(\Gamma_{1 / 2-\gamma} \times \mathbb{R}^{l}\right) \rightarrow S^{-\infty}\left(\Gamma_{1 / 2-\gamma} \times \mathbb{R}^{l}\right)=\mathscr{S}\left(\Gamma_{1 / 2-\gamma} \times \mathbb{R}^{l}\right) .
$$

In particular,

$$
\chi(s) m(f)(s, \lambda) \in \mathscr{T}^{\gamma}\left(\mathbb{R}_{+, s}, \mathscr{S}\left(\mathbb{R}_{\lambda}^{l}\right)\right)
$$

for every $f(1 / 2-\gamma+i \rho, \lambda) \in S_{(\mathrm{cl})}^{\mu}\left(\Gamma_{1 / 2-\gamma} \times \mathbb{R}^{l}\right)$. Here $\mathscr{T}^{\gamma}\left(\mathbb{R}_{+, s}\right):=M_{\gamma}^{-1} \mathscr{S}\left(\Gamma_{\frac{1}{2}-\gamma}\right) ;$ the former $\mathscr{T}\left(\mathbb{R}_{+, s}\right)$ corresponds to $\mathscr{T}^{1 / 2}\left(\mathbb{R}_{+, s}\right)$. 
Definition 4. Let $S_{(\mathrm{cl})}^{\mu}\left(\mathbb{C} \times \mathbb{R}^{l}\right)$ denote the space of all $h(z, \lambda) \in \mathscr{A}\left(\mathbb{C}, S_{(\mathrm{cl})}^{\mu}\left(\mathbb{R}_{\lambda}^{l}\right)\right)$ such that

$$
\left.h\right|_{\Gamma_{\delta} \times \mathbb{R}^{l}} \in S_{(\mathrm{cl})}^{\mu}\left(\Gamma_{\delta} \times \mathbb{R}^{l}\right)
$$

for every $\delta \in \mathbb{R}$, uniformly in compact $\delta$-intervals.

The spaces $S_{(\mathrm{cl})}^{\mu}\left(\mathbb{C} \times \mathbb{R}^{l}\right)$ are Fréchet in a natural way.

Recall that for any $K \Subset \mathbb{R}_{+}$we set $C_{0}^{\infty}(K):=\left\{u \in C^{\infty}\left(\mathbb{R}_{+}\right): \operatorname{supp} u \subseteq K\right\}$.

Theorem 7. For every $K \Subset \mathbb{R}_{+}$the kernel cut-off operator $V_{M}:(\varphi, f) \rightarrow V_{M}(\varphi) f$ induces a bilinear continuous mapping

$$
\boldsymbol{V}_{M}: C_{\mathbf{0}}^{\infty}(K) \times S_{(\mathrm{cl})}^{\mu}\left(\Gamma_{\beta} \times \mathbb{R}_{\lambda}^{l}\right) \rightarrow S_{(\mathrm{cl})}^{\mu}\left(\mathbb{C} \times \mathbb{R}^{l}\right) .
$$

for any $\beta \in \mathbb{R}$.

Proof. For simplicity we consider the case $\beta=0$. Let us first go back to the analogue of the formula (3.50), namely,

$$
V_{M}(\varphi) f(i \rho, \lambda)=\int s^{i \rho} \varphi(s)\left\{\int s^{-i \rho^{\prime}} f\left(i \rho^{\prime}, \lambda\right) d s / s\right\} d \rho^{\prime} .
$$

In this case we have

$$
m(f)(s, \lambda)=\int s^{-i \rho^{\prime}} f\left(i \rho^{\prime}, \lambda\right) d s / s \in \mathscr{T}^{\prime}\left(\mathbb{R}_{\theta, \lambda}^{1+l}\right),
$$

and $\varphi(s) k_{M}(f)(s, \lambda)$ has compact support with respect to $s$. Thus

$$
V_{M}(\varphi) f(i \rho, \lambda)=\int s^{i \rho} \varphi(s)
$$

$m(f)(s, \lambda) d s / s$ extends to a function

$$
\boldsymbol{V}_{M}(\varphi) f(\delta+i \rho, \lambda)=\int s^{\delta+i \rho} \varphi(s) m(f)(s, \lambda) d s / s
$$

which is holomorphic in $w=\delta+i \rho \in \mathbb{C}$. The arguments are analogous to the corresponding case of Fourier transforms of scalar distributions with compact support. We have

$$
h(\delta+i \rho, \lambda):=V_{M}(\varphi) f(\delta+i \rho, \lambda)=V_{M}\left(\varphi_{\delta}\right) f(i \rho, \lambda)
$$

for $\varphi_{\delta}(s):=s^{\delta} \varphi(s) \in C_{\mathbf{0}}^{\infty}(K)$. From (3.50) we have $\boldsymbol{V}_{M}(\varphi) f(\delta+i \rho, \lambda) \in S_{(\mathrm{cl})}^{\mu}\left(\Gamma_{\delta} \times \mathbb{R}_{\lambda}^{l}\right)$ for every $\delta$. Since $\delta \rightarrow \varphi_{\delta}$ represents a continuous mapping $\mathbb{R} \rightarrow C_{0}^{\infty}(K)$, the continuity of

$$
V_{M}: C_{\mathbf{0}}^{\infty}(K) \times S_{(\mathrm{cl})}^{\mu}\left(\Gamma_{0} \times \mathbb{R}_{\lambda}^{l}\right) \rightarrow S_{(\mathrm{cl})}^{\mu}\left(\Gamma_{\delta} \times \mathbb{R}_{\lambda}^{l}\right)
$$

implies that (3.54) is uniform in compact $\delta$-intervals. The closed graph theorem gives us also the continuity of (3.55) with respect to the Fréchet topology of the space $S_{(\mathrm{cl})}^{\mu}\left(\mathbb{C} \times \mathbb{R}^{l}\right)$.

The following results can be proved in an analogous manner as those in the final part of Subsection 3.1 . 
Corollary 10. Let $\psi \in C_{0}^{\infty}\left(\mathbb{R}_{+}\right)$be a cut-off function, and $f(z, \lambda) \in S_{(\mathrm{cl})}^{\mu}\left(\Gamma_{\delta} \times \mathbb{R}^{l}\right)$. Then we have

and

$$
h(z, \lambda):=V_{M}(\psi) f(z, \lambda) \in S_{(\mathrm{cl})}^{\mu}\left(\mathbb{C} \times \mathbb{R}^{l}\right)
$$

$$
\left.h(z, \lambda)\right|_{\Gamma_{\delta} \times \mathbb{R}^{l}}=f(z, \lambda) \bmod S^{-\infty}\left(\Gamma_{\delta} \times \mathbb{R}^{l}\right)
$$

Corollary 11. Let $\varphi \in C_{0}^{\infty}\left(\mathbb{R}_{+}\right)$and define

$$
h_{\delta}(i \rho, \lambda):=\left(V_{M}(\varphi) f\right)(\delta+i \rho, \lambda)
$$

for any fixed $\delta \in \mathbb{R}$. Then $f \rightarrow h_{\delta}$ defines a continuous map

$$
S_{(\mathrm{cl})}^{\mu}\left(\Gamma_{0} \times \mathbb{R}^{l}\right) \rightarrow S_{(\mathrm{cl})}^{\mu}\left(\Gamma_{0} \times \mathbb{R}^{l}\right)
$$

and we have

$$
h_{0}(i \rho, \lambda)=h_{\delta}(i \rho, \lambda) \bmod S_{(\mathrm{cl})}^{\mu-1}\left(\Gamma_{0} \times \mathbb{R}^{l}\right) .
$$

Lemma 3. Let $h(z, \lambda) \in S_{(\mathrm{cl})}^{\mu}\left(\mathbb{C} \times \mathbb{R}^{l}\right)$, and set $h_{\delta}(i \rho, \lambda):=h(\delta+i \rho, \lambda)$. Then the mapping, $\mathbb{R} \rightarrow$ $S_{(\mathrm{cl})}^{\mu}\left(\Gamma_{0} \times \mathbb{R}^{l}\right), \delta \rightarrow h_{\delta}$, is continuous.

Proposition 4. For every $h(z, \lambda) \in S_{(\mathrm{cl})}^{\mu}\left(\mathbb{C} \times \mathbb{R}^{l}\right)$ we have

$$
\left(\partial_{z} h\right)(z, \lambda) \in S_{(\mathrm{cl})}^{\mu-1}\left(\mathbb{C} \times \mathbb{R}^{l}\right)
$$

(here $\partial_{z}$ is the differentiation of $h$ as a holomorphic function with values in $S_{(\mathrm{cl})}^{\mu}\left(\mathbb{R}^{l}\right)$ ).

Theorem 8. Let $h(z, \lambda) \in S_{(\mathrm{cl})}^{\mu}\left(\mathbb{C} \times \mathbb{R}^{l}\right)$ and fix $\delta \in \mathbb{R} ;$ then

(i) $h_{\delta} \in S_{(\mathrm{cl})}^{\mu-\varepsilon}\left(\Gamma_{\delta} \times \mathbb{R}^{l}\right)$ for some $\varepsilon \geq 0(\varepsilon \in \mathbb{N}$ in the classical case $)$ implies $h \in S_{(\mathrm{cl})}^{\mu-\varepsilon}\left(\mathbb{C} \times \mathbb{R}^{l}\right)$;

(ii) $h_{\delta} \in S_{\mathrm{cl}}^{\mu}\left(\Gamma_{\delta} \times \mathbb{R}^{l}\right)$ implies $h \in S_{\mathrm{cl}}^{\mu}\left(\mathbb{C} \times \mathbb{R}^{l}\right)$.

\subsection{Mellin quantization}

By Mellin quantisation we understand the representation of Fourier by Mellin pseudodifferential operators. This does not concern the push forward of an operator under the diffeomorphism $\mathbb{R} \rightarrow \mathbb{R}_{+}, t \mapsto e^{-t}$, but the shape of Fourier-based pseudo-differential operators on the $r$ half-axis as an open subset of $\mathbb{R}$ in terms of the Mellin transform with respect to the same half-axis coordinate. In Subsection 2.2 we discussed aspects of such a change for differential operators. From the mere pseudo-differential viewpoint the change from the Fourier to the Mellin picture is nothing else than a change of the phase function from $\left(r-r^{\prime}\right) \rho$ to $\left(\log r^{\prime}-\log r\right) \rho$. However, the control of symbols is far from being completely trivial, since such a change produces (smoothing) remainders with a singular behaviour at $r=0$ that can affect (or destroy) a desired mapping property of operators close to $r=0$. 
Let us now give a striking example for "unexpected" effects that appear under replacing the Fourier by the Mellin transform in pseudo-differential operators in dimension 1. First consider operators

$$
\mathrm{Op}(a) u(r)=\iint e^{i\left(r-r^{\prime}\right) \rho} a(\rho) u\left(r^{\prime}\right) d r^{\prime} d \rho
$$

for symbols $a(\rho) \in S_{\mathrm{cl}}^{0}(\mathbb{R})$. Let us form the truncation of $\mathrm{Op}(a): L^{2}(\mathbb{R}) \rightarrow L^{2}(\mathbb{R})$ to the half-axis

$$
\mathrm{Op}^{+}(a):=\mathrm{r}^{+} \mathrm{Op}(a) \mathrm{e}^{+}: L^{2}\left(\mathbb{R}_{+}\right) \rightarrow L^{2}\left(\mathbb{R}_{+}\right)
$$

where $\mathrm{e}^{+}: L^{2}\left(\mathbb{R}_{+}\right) \rightarrow L^{2}(\mathbb{R})$ extends functions by zero from $\mathbb{R}_{+}$to $\mathbb{R}$ and $\mathrm{r}^{+}: L^{2}(\mathbb{R}) \rightarrow L^{2}\left(\mathbb{R}_{+}\right)$ restricts to $\mathbb{R}_{+}$. Eskin in his book [18] found out a Mellin representation of (3.63) locally at $r=0$ in terms of Mellin pseudo-differential operators with meromorphic Mellin symbols, modulo Hilbert-Schmidt operators, including the behaviour under compositions and the nature of parametrices for elliptic operators. Later on this structure has been deepened in different directions, see, in particular, [36], [37], [38], and also Theorem 3.64 below.

The half-axis $\overline{\mathbb{R}}_{+} \in \mathfrak{M}_{1}$ is a manifold with conical singularity $r=0$ and conical exit to infinitiy $r \rightarrow \infty$. We then have the cone algebra over $\overline{\mathbb{R}}_{+}$which is in its simplest form furnished by zero-order classical pseudo-differential operators

$$
A=\omega \mathrm{Op}_{M}(h) \omega^{\prime}+(1-\omega) A_{\mathrm{int}}\left(1-\omega^{\prime \prime}\right)+M+G,
$$

$\mathrm{Op}_{M}(\cdot)=\mathrm{Op}_{M}^{0}(\cdot)$, elements $h(r, z) \in C^{\infty}\left(\overline{\mathbb{R}}_{+}, S_{\mathrm{cl}}^{0}(\mathbb{C})\right)$, cut-off functions $\omega^{\prime \prime}<\omega \prec \omega^{\prime}$ on the halfaxis, $A_{\mathrm{int}} \in L_{\mathrm{cl}}^{0}\left(\mathbb{R}_{+}\right)$with exit property of order 0 at $r=\infty$, and smoothing Mellin plus Green operators $M+G$ with discrete asymptotics at $r=0$. The operator $M$ is essentially a smoothing Mellin operator with meromorphic symbol, not necessarily compact in $L^{2}\left(\mathbb{R}_{+}\right)$, while the Green operator is an asymptotics generating compact smoothing operator. The notation comes from Green's function in solution formulas of boundary value problems. A specific part of such operators is a pseudo-differential operator locally along the boundary with Green operator-valued symbol. In Sections 4 and 5 below we encounter Green operators for boundary value problems with the transmission property at the boundary. This calculus, cf. Boutet de Monvel [7], and the monograph [35], is also based on the truncation convention, using the operators $\mathrm{e}^{+}$and $\mathrm{r}^{+}$in direction of the inner normal $\overline{\mathbb{R}}_{+}$.

Theorem 9. [44] The operator (3.63) is an element of the cone algebra on $\mathbb{R}_{+}$where the half-axis is regarded as a manifold with conical singularity, the origin.

A similar truncation result holds on a manifold $M$ with boundary of dimension $>0$. In this case $M$ is interpreted as a manifold with edge, where the edge is just the boundary. The pseudo-differential calculus of boundary value problems on $M$ is then a special case of the 
edge calculus. The corresponding operators are more general than those with the transmission property at the boundary. Typical examples of operators of the latter kind will be discussed later on in this exposition. Those are of independent interest. The tools for the case without the transmission property and the edge calculus in general are another chapter of the singular analysis. We will develop step by step more details on these operator structures. Let us only recall for the moment that the corresponding algebras are designed to express parametrices of elliptic operators within the calculus. In particular, this is the case for the edge algebra, with its two-component principal symbolic structure $\left(\sigma_{0}, \sigma_{1}\right)$, where $\sigma_{1}$ takes values in the above-mentioned cone algebra.

Theorem 10 ([47]). Let $M$ be a manifold with smooth boundary, $2 M$ it double, and $\tilde{A}$ a classical pseudo-differential operator on $2 M$, say, of order zero, and let $A:=\mathrm{r}^{+} \tilde{A} \mathrm{e}^{+}$be the corresponding truncated operator with $\mathrm{e}^{+}$being the extension by zero from int $M$ to $2 M$ and $\mathrm{r}^{+}$the restriction to int $M$. Then $A$ is an element of the edge algebra on $M$ where the boundary is interpreted as edge.

We now outline a few aspects of the Mellin quantisation in concise form.

Let us set

$$
M\left(r, r^{\prime}\right):= \begin{cases}r^{-1} & \text { for } r=r^{\prime}, \\ \left(r-r^{\prime}\right)^{-1}\left(\log r-\log r^{\prime}\right) & \text { for } r \neq r^{\prime} .\end{cases}
$$

Then we have $M\left(r, r^{\prime}\right) \in C^{\infty}\left(\mathbb{R}_{+} \times \mathbb{R}_{+}\right)$and $M\left(r, r^{\prime}\right)>0$ for every $\left(r, r^{\prime}\right) \in \mathbb{R}_{+} \times \mathbb{R}_{+}$.

Lemma 4. For every $k \in \mathbb{N} \backslash\{0\}$ we have

$$
\left.\partial_{r^{\prime}}^{k} M\left(r, r^{\prime}\right)\right|_{r^{\prime}=r}=c_{k} r^{-k-1}
$$

for suitable constants $c_{k} \in \mathbb{R}$, in particular,

$$
\left(\left.r^{\prime} \partial_{r^{\prime}}{ }^{k}\left\{r^{\prime} M\left(r, r^{\prime}\right)\right\}\right|_{r^{\prime}=r} \in C^{\infty}\left(\overline{\mathbb{R}}_{+}\right), \quad \text { and }\left.\quad r^{k-1} \partial_{r^{\prime}}^{k}\left\{M\left(r, r^{\prime}\right)^{-1}\right\}\right|_{r^{\prime}=r} \in C^{\infty}\left(\overline{\mathbb{R}}_{+}\right) .\right.
$$

Proof. Set $1+x=r / r^{\prime},|x|<1$ for $r, r^{\prime} \in \mathbb{R}_{+}$. Then

$$
\log r-\log r^{\prime}=\log (1+x)=\sum_{j=1}^{\infty} \frac{(-1)^{j+1}}{j} x^{j}=\sum_{j=1}^{\infty} \frac{(-1)^{j+1}}{j} \frac{\left(r-r^{\prime}\right)^{j}}{r^{\prime j}} .
$$

Thus

$$
M\left(r, r^{\prime}\right)=\frac{\log r-\log r^{\prime}}{r-r^{\prime}}=\sum_{k=0}^{\infty} \frac{(-1)^{k}}{k+1} \frac{\left(r-r^{\prime}\right)^{k}}{r^{\prime k+1}} .
$$

It follows that $\left.\partial_{r}^{k} M\left(r, r^{\prime}\right)\right|_{r=r^{\prime}}=k !(-1)^{k}(k+1)^{-1} r^{\prime-k-1}$ which gives us (3.66). Then the first relation of (3.67) follows from Remark 6. By induction we see that $\partial_{r^{\prime}}^{k}\left(M\left(r, r^{\prime}\right)^{-1}\right)$ is a linear 
combination of expressions $M\left(r, r^{\prime}\right)^{-m-1} \prod_{l=1}^{m} \partial_{r^{\prime}}^{j_{l}} M\left(r, r^{\prime}\right)$ where $m \leq k$ and $\sum_{l=1}^{m} j_{l}=k$. Thus $\partial_{r^{\prime}}^{k}\left(\left.M\left(r, r^{\prime}\right)^{-1}\right|_{r^{\prime}=r}\right.$ is a linear combination of terms $r^{m+1} r^{-m-k}, 0 \leq m \leq k$, which show the second relation of (3.67).

Given any $a(\xi, \lambda) \in S^{\mu}\left(\mathbb{R}^{1+l}\right)$ and $\varphi(\theta) \in C_{0}^{\infty}\left(\mathbb{R}_{+}\right)$we form

$$
\left(Q_{M}(\varphi) a\right)(i \rho, \lambda):=\int_{\mathbb{R}} \int_{\mathbb{R}} e^{-i s \xi} e^{i \xi} s^{-i \rho} \varphi(s) a(\xi, \lambda) d s d \xi .
$$

Moreover, for any $f(i \rho, \lambda) \in S^{\mu}\left(\Gamma_{0} \times \mathbb{R}^{l}\right)$ and $\psi(s) \in C_{0}^{\infty}\left(\mathbb{R}_{+}\right)$we form

$$
\left(Q_{F}(\psi) f\right)(\xi, \lambda):=\int_{-\infty}^{\infty} \int_{0}^{\infty} s^{i \tau} e^{-i \xi} e^{i s \xi} \psi(s) f(i \tau, \lambda) \frac{d s}{s} d \tau .
$$

Theorem 11. (i) The expression (3.68) yields a separately continuous mapping

$$
Q_{M}: C_{0}^{\infty}\left(\mathbb{R}_{+}\right) \times S_{(\mathrm{cl})}^{\mu}\left(\mathbb{R}^{1+l}\right) \rightarrow S_{(\mathrm{cl})}^{\mu}\left(\Gamma_{0} \times \mathbb{R}^{l}\right),(\varphi, a) \rightarrow Q_{M}(\varphi) a .
$$

There are coefficients $c_{k j}(\varphi)$ (only depending on $\left(\partial_{s}^{l} \varphi\right)(1), l \in \mathbb{N}$, but not on the symbol a) such that

$$
Q_{M}(\varphi) a(i \rho, \lambda) \sim \varphi(1) a(-\rho, \lambda)+\sum_{k=1}^{\infty} \sum_{j=0}^{k} c_{k j}(\varphi)(-\rho)^{j}\left(\partial_{\rho}^{k+j} a\right)(-\rho, \lambda),
$$

$(\rho, \lambda) \in \mathbb{R}^{1+l}$.

(ii) The operator (3.69) yields a separately continuous mapping

$$
Q_{F}: C_{0}^{\infty}\left(\mathbb{R}_{+}\right) \times S_{(\mathrm{cl})}^{\mu}\left(\Gamma_{0} \times \mathbb{R}^{l}\right) \rightarrow S_{(\mathrm{cl})}^{\mu}\left(\mathbb{R} \times \mathbb{R}^{l}\right),(\psi, f) \rightarrow Q_{F}(\psi) f .
$$

There are coefficients $d_{k j}(\psi)$ (only depending on $\left(\partial_{s}^{l} \psi\right)(1), l \in \mathbb{N}$, but not on the symbol $f$ ) such that

$$
\left(Q_{F}(\psi) f\right)(\xi, \lambda) \sim \psi(1) f(i \xi, \lambda)+\sum_{k=1}^{\infty} \sum_{j=0}^{k} d_{k j}(\psi)(-i \xi)^{j}\left(\partial_{\xi}^{k+j} f\right)(-i \xi, \lambda),
$$

$(\xi, \lambda) \in \mathbb{R}^{1+l}$

(iii) If $\varphi$ and $\psi$ are equal to 1 in a neighbourhood of 1 , then we have

$$
\begin{aligned}
& Q_{M}(\varphi) Q_{F}(\psi) f=f \bmod S^{-\infty}\left(\Gamma_{0} \times \mathbb{R}^{l}\right), \\
& Q_{F}(\psi) Q_{M}(\varphi) a=a \bmod S^{-\infty}\left(\mathbb{R} \times \mathbb{R}^{l}\right) .
\end{aligned}
$$

Proof. We will show that $Q_{M}$ and $Q_{F}$ generate linear mappings (3.70) and (3.72), respectively, and we will obtain the separate continuity. 
We mainly look at general symbols. Then the asserted asymptotic expansion (3.71) shows that $Q_{M}$ transforms classical symbols to classical ones. Using the function (3.65) and substituting $\xi \mapsto-M(s, 1)(\rho+\xi)=(1-s)^{-1} \log s(\rho+\xi)$, taking into account that the amplitude function has compact support in $s$, it follows that

$$
Q_{M}(\varphi) a(i \rho, \lambda)=\int_{\mathbb{R}} \int_{0}^{\infty} s^{i \xi} \varphi(s) s M(s, 1) a(-M(s, 1)(\rho+\xi), \lambda) \frac{d s}{s} d \xi
$$

In fact, replacing $\xi$ in $e^{-i s \xi} s^{-i \rho}$ by $-s^{-1} \log s(\rho+\xi)$ gives us for the first factor $e^{-i s \xi}=e^{-i s\left(-s^{-1} \log s(\rho+\xi)\right)}=e^{i(\log s(\rho+\xi))}=s^{i \rho} s^{i \xi}$. The regularisation of the oscillatory integral (3.73) gives us a symbol in $S^{\mu}\left(\Gamma_{0} \times \mathbb{R}^{q}\right)$. Let us now derive the asymptotic expansion (3.71). From Taylor's formula, applied to $a(M(s, 1)(\rho+\xi, \lambda))$ with respect to $\xi$ at $\xi=0$ under the integral in (3.73) we obtain for every $N \in \mathbb{N}$

$$
\left(Q_{M}(\varphi) a\right)(i \rho, \eta)=\sum_{k=0}^{N} b_{k}(i \rho, \lambda)+r_{N+1}(i \rho, \lambda)
$$

where

$$
\begin{aligned}
b_{k}(i \rho, \lambda)= & \frac{(-1)^{k}}{k !} \int_{\mathbb{R}} \int_{0}^{\infty} s^{i \xi} \xi^{k} \varphi(s) s M(s, 1)^{k+1}\left(\partial_{\xi}^{k} a\right)(-M(s, 1) \rho, \lambda) \frac{d s}{s} d \xi, \\
r_{N+1}(i \rho, \lambda)= & \int_{0}^{1} \frac{(1-t)^{N}}{N !}(-1)^{N+1} \int_{\mathbb{R}} \int_{0}^{\infty} s^{i \xi} \xi^{N+1} \varphi(s) s M(s, 1)^{N+2} \\
& \left(\partial_{\xi}^{N+1} a\right)\left(-M(s, 1)(\rho+t \xi, \lambda) \frac{d s}{s} d \xi d t .\right.
\end{aligned}
$$

Using standard properties of oscillatory integrals we obtain $r_{N+1}(i \rho, \lambda) \in S^{\mu-(N+1)}\left(\Gamma_{0} \times \mathbb{R}^{l}\right)$ and

$$
b_{k}(i \rho, \lambda)=\left.\frac{(-1)^{k}}{k !}\left(s^{\prime} \partial_{s^{\prime}}\right)^{k}\left\{\varphi\left(s^{\prime}\right) s^{\prime} M\left(s^{\prime}, 1\right)^{k+1}\left(\partial_{\xi}^{k} a\right)\left(-M\left(s^{\prime}, 1\right) \rho, \lambda\right)\right\}\right|_{s^{\prime}=1} .
$$

which implies the claimed asymptotic expansion.

The proof of (ii) is analogous to (i) when we employ that $Q_{F}(\psi) f$ can be written in the form

$$
\left(Q_{F}(\psi) f\right)(\xi, \lambda)=\int_{\mathbb{R}} \int_{\mathbb{R}_{+}} r^{-i \tau} e^{-i \xi} e^{-i r \xi} \psi(r) M(r, 1)^{-1} f\left(-M(s, 1)^{-1}(i(\xi+\tau)), \lambda\right) \frac{d r}{r} d \tau .
$$

Finally, for (iii) we observe that, using compact supports in $s$ and $r$ in the respective oscillatory integrals,

$$
\begin{aligned}
Q_{M} Q_{F} f(i \rho, \lambda) & =\iint e^{-i s \xi} e^{i \xi} s^{-i \rho} \varphi(s)\left\{\iint r^{i \tau} e^{i r \xi} e^{-i \xi} \psi(r) f(i \tau, \lambda) \frac{d r}{r} d \tau\right\} d s d \xi \\
& =\iint s^{-i \rho} \varphi(s)\left(\int e^{-i s \xi}\left[\int e^{i r \xi} \psi(r) r^{i \tau} \frac{d r}{r}\right] d \xi\right) f(i \tau, \lambda) d \tau d s
\end{aligned}
$$




$$
\begin{aligned}
& =\int s^{-i \rho} \varphi(s)\left(F_{\xi \rightarrow s} F_{r \rightarrow \xi}^{-1}\left[r^{-1} \psi(r) r^{i \tau}\right]\right) f(i \tau, \lambda) d \tau d s \\
& =\iint s^{-i \rho} \varphi(s) \psi(s) s^{i \tau} f(i \tau, \lambda) d \tau \frac{d s}{s}=\iint r^{i(\rho-\tau)}(\varphi \psi)\left(r^{-1}\right) f(i \tau, \lambda) d \tau \frac{d r}{r} \\
& =\int_{\mathbb{R}} \int_{0}^{\infty} r^{i \tilde{\rho}}(\varphi \psi)\left(r^{-1}\right) f(i(\rho-\tilde{\rho}), \lambda) d \tilde{\rho} \frac{d r}{r}=V_{M}\left(\phi_{M}\right) f(i \rho, \lambda)
\end{aligned}
$$

with $V_{M}\left(\phi_{M}\right)$ being the kernel cut-off operator for $\phi_{M}(r):=(\varphi \psi)\left(r^{-1}\right)$, cf. (3.45). In an analogous manner we can show that

$$
\begin{aligned}
Q_{F}(\psi) Q_{M}(\varphi) a(\xi, \lambda) & =\int_{\mathbb{R}} \int_{\mathbb{R}} e^{-i \theta \rho}(\varphi \psi)(-\theta+1) a(\xi-\rho, \lambda) d \theta d \rho \\
& =V_{F}\left(\phi_{F}\right) a(\xi, \lambda)
\end{aligned}
$$

which is the Fourier kernel cut-off operator with the cut-off function $\phi_{F}(\theta)=(\varphi \psi)(-\theta+1)$, see the formulas (3.44), (3.45) and (3.14), respectively.

\section{Remark 12.}

(i) Let $\varphi_{1}, \varphi_{2}$ and $\psi_{1}, \psi_{2}$ be functions in $C_{0}^{\infty}\left(\mathbb{R}_{+}\right)$that are equal to 1 in a neighbourhood of 1. Then for every $a(\rho, \eta) \in S^{\mu}\left(\mathbb{R}^{1+l}\right)$ we have

$$
Q_{M}\left(\varphi_{1}\right) a=Q_{M}\left(\varphi_{2}\right) a \bmod S^{-\infty}\left(\Gamma_{0} \times \mathbb{R}^{l}\right),
$$

and for every $f(z, \eta) \in S^{\mu}\left(\Gamma_{0} \times \mathbb{R}^{l}\right)$

$$
Q_{F}\left(\psi_{1}\right) f=Q_{F}\left(\psi_{2}\right) f \bmod S^{-\infty}\left(\mathbb{R} \times \mathbb{R}^{l}\right) .
$$

(ii) If $\varphi$ and $\psi$ are functions in $C_{0}^{\infty}\left(\mathbb{R}_{+}\right)$that are equal to 1 in a neighbourhood of 1 , then for every $\mu \in \mathbb{R}$ the mappings $Q_{M}(\varphi)$ and $Q_{F}(\psi)$ induce isomorphisms

$$
Q_{M}(\varphi): S_{(\mathrm{cl})}^{\mu}\left(\mathbb{R}^{1+l}\right) / S^{-\infty}\left(\mathbb{R}^{1+l}\right) \rightarrow S_{(\mathrm{cl})}^{\mu}\left(\Gamma_{0} \times \mathbb{R}^{l}\right) / S^{-\infty}\left(\left(\Gamma_{0} \times \mathbb{R}^{l}\right)\right.
$$

and

$$
Q_{F}(\psi): S_{(\mathrm{cl})}^{\mu}\left(\Gamma_{0} \times \mathbb{R}^{l}\right) / S^{-\infty}\left(\Gamma_{0} \times \mathbb{R}^{l}\right) \rightarrow S_{(\mathrm{cl})}^{\mu}\left(\mathbb{R} \times \mathbb{R}^{l}\right) / S^{-\infty}\left(\mathbb{R} \times \mathbb{R}^{l}\right),
$$

respectively.

Theorem 12. Let $\varphi, \psi \in C_{0}^{\infty}\left(\mathbb{R}_{+}\right)$be equal to 1 near 1 .

(i) For $a(r, \rho, \lambda):=\tilde{a}(r, r \rho, \lambda), \tilde{a}(r, \tilde{\rho}, \lambda) \in C^{\infty}\left(\mathbb{R}_{+}, S^{\mu}\left(\mathbb{R}_{\tilde{\rho}, \lambda}^{1+l}\right)\right)$, and

$$
f(r, i \rho, \lambda):=\left(Q_{M}(\varphi) \tilde{a}\right)(r, i \rho, \lambda)
$$

we have

$$
\mathrm{Op}_{r}(a)(\lambda)-\mathrm{Op}_{M}^{1 / 2}(f)(\lambda)=\mathrm{Op}_{r}\left(\left(1-\varphi\left(\frac{r^{\prime}}{r}\right)\right) a\right)(\lambda)
$$

as operators $C_{0}^{\infty}\left(\mathbb{R}_{+}\right) \rightarrow C^{\infty}\left(\mathbb{R}_{+}\right)$. 
(ii) Let $f(r, i \rho, \lambda) \in C^{\infty}\left(\mathbb{R}_{+}, S^{\mu}\left(\Gamma_{0} \times \mathbb{R}^{l}\right)\right)$, and

$$
a(r, \rho, \eta):=Q_{F}(\psi) f(r, r \rho, \lambda) .
$$

Then we have

$$
\mathrm{Op}_{M}^{1 / 2}(f)(\lambda)-\mathrm{Op}_{r}(a)(\lambda)=\mathrm{Op}_{M}^{1 / 2}\left(\left(1-\psi\left(\frac{r^{\prime}}{r}\right)\right) f\right)(\lambda)
$$

as operators $C_{0}^{\infty}\left(\mathbb{R}_{+}\right) \rightarrow C^{\infty}\left(\mathbb{R}_{+}\right)$.

Proof. (i) Let us write

$$
a(r, \rho, \lambda)=\varphi\left(\frac{r^{\prime}}{r}\right) a(r, \rho, \lambda)+\left(1-\varphi\left(\frac{r^{\prime}}{r}\right)\right) a(r, \rho, \lambda) .
$$

Then the assertion is proved if we show that

$$
\mathrm{Op}_{r}\left(\varphi\left(\frac{r^{\prime}}{r}\right) a\right)(\lambda)=\mathrm{Op}_{M}^{1 / 2}(f)(\lambda)
$$

as operators $C_{0}^{\infty}\left(\mathbb{R}_{+}\right) \rightarrow C^{\infty}\left(\mathbb{R}_{+}\right)$. For $u \in C_{0}^{\infty}\left(\mathbb{R}_{+}\right)$we have

$$
\begin{aligned}
\mathrm{Op}_{r}\left(\varphi\left(\frac{r^{\prime}}{r}\right) a\right)(\lambda) u(r) & =\int_{\mathbb{R}} \int_{0}^{\infty} e^{i\left(r-r^{\prime}\right) \rho} \varphi\left(\frac{r^{\prime}}{r}\right) a(r, \rho, \lambda) u\left(r^{\prime}\right) d r^{\prime} d \rho \\
& =\int_{\mathbb{R}} \int_{0}^{\infty}\left(\frac{r}{r^{\prime}}\right)^{-i \rho} D\left(r, r^{\prime}, i \rho, \lambda\right) u\left(r^{\prime}\right) \frac{d r^{\prime}}{r^{\prime}} d \rho=\mathrm{Op}_{M}^{\frac{1}{2}}(m)(\lambda) u(r)
\end{aligned}
$$

for $D\left(r, r^{\prime}, i \rho, \lambda\right):=M\left(r, r^{\prime}\right) r^{\prime} \varphi\left(r^{\prime} / r\right) a\left(r,-M\left(r, r^{\prime}\right) \rho, \lambda\right)$. Here we substituted $\tau=-M\left(r, r^{\prime}\right) \rho$ and then replaced again $\tau$ by $\rho$.

We now define

$$
f(r, \tau, \lambda):=r^{i \tau}\left(\mathrm{Op}_{M}^{\frac{1}{2}}(D)(\lambda)\left(r^{\prime}\right)^{-i \tau}\right)(r)=\int_{\mathbb{R}} \int_{0}^{\infty} s^{i \xi} D(r, s r, i(\xi+\tau), \lambda) \frac{d s}{s} d \xi .
$$

The right hand side of (3.80) follows from $r^{i \tau} \iint r^{-i \rho} m\left(r, r^{\prime}, i \rho, \eta\right) r^{\prime i \rho} r^{\prime-i \tau} d r^{\prime} / r^{\prime} d \rho$ for the expression in the middle and the substitutions $s=r^{\prime} / r, \xi=\rho-\tau$. Observe that (3.80) makes sense since the factor $\varphi\left(r^{\prime} / r\right)$ produces a properly supported operator. Then we have $O p_{M}^{\frac{1}{2}}(D)(\lambda)=$ $\mathrm{Op}_{M}^{\frac{1}{2}}(f)(\lambda)$, cf. the formula (2.62) for $\gamma=1 / 2$. From

$$
M\left(r, r^{\prime}\right)=r^{-1}\left(1-r / r^{\prime}\right)^{-1} \log \left(r / r^{\prime}\right)
$$

we obtain $M(r, s r)=r^{-1}(1-s)^{-1} \log (1 / s)$ and $M(t, 1)=t^{-1}(1-1 / t)^{-1} \log t$. Thus

$$
\begin{gathered}
\int_{\mathbb{R}} \int_{0}^{\infty} s^{i \xi} D(r, s r, i(\xi+\tau), \lambda) \frac{d s}{s} d \xi=\int_{\mathbb{R}} \int_{0}^{\infty} s^{i \xi} r^{-1}(1-s)^{-1} \log (1 / s) \operatorname{sr} \varphi(s) \\
\tilde{a}\left(r,-(1-s)^{-1} \log (1 / s)(\xi+\tau), \lambda\right) \frac{d s}{s} d \xi=Q_{M}(\varphi) \tilde{a}(r, i \tau, \lambda),
\end{gathered}
$$


cf. (3.73). In other words, we proved the relation (3.77).

(ii) Analogously as (3.78) we write

$$
f(r, i \tau, \lambda)=\psi\left(\frac{r^{\prime}}{r}\right) f(r, i \tau, \lambda)+\left(\left(1-\psi\left(\frac{r^{\prime}}{r}\right)\right) f(r, i \tau, \lambda)\right.
$$

which reduces the assertion to $\mathrm{Op}_{M}^{\gamma}\left(\psi\left(r^{\prime} / r\right) f\right)(\lambda):=\mathrm{Op}_{r}(a)(\lambda)$ as $C_{0}^{\infty}\left(\mathbb{R}_{+}\right) \rightarrow C^{\infty}\left(\mathbb{R}_{+}\right)$. For $u \in C_{0}^{\infty}\left(\mathbb{R}_{+}\right)$we have

$$
\begin{aligned}
\operatorname{Op}_{M}^{1 / 2}\left(\psi\left(\frac{r^{\prime}}{r}\right) f\right)(\lambda) u(r) & =\int_{\mathbb{R}} \int_{0}^{\infty}\left(\frac{r}{r^{\prime}}\right)^{-i \tau} \psi\left(\frac{r^{\prime}}{r}\right) f(r, i \tau, \lambda) u\left(r^{\prime}\right) \frac{d r^{\prime}}{r^{\prime}} d \tau \\
& =\int_{\mathbb{R}} \int_{0}^{\infty} e^{i\left(r-r^{\prime}\right) \tau} g\left(r, r^{\prime}, \tau, \lambda\right) u\left(r^{\prime}\right) d r^{\prime} d \tau,
\end{aligned}
$$

for

$$
g\left(r, r^{\prime}, \tau, \lambda\right):=\left(r^{\prime} M\left(r, r^{\prime}\right)\right)^{-1} \psi\left(\frac{r^{\prime}}{r}\right) f\left(r,-M\left(r, r^{\prime}\right)^{-1} i \tau, \lambda\right) .
$$

Because of the factor $\psi\left(r^{\prime} / r\right)$ the operator $\mathrm{Op}_{r}(g)(\lambda)$ is properly supported, and hence, when we form

$$
a(r, \tau, \lambda):=e^{-i r \tau}\left(\operatorname{Op}_{r}(g)(\lambda) e^{i r^{\prime} \tau}\right)(r)=\int_{\mathbb{R}} \int_{0}^{\infty} e^{-i\left(r^{\prime}-1\right) \xi} g\left(r, r r^{\prime}, \frac{\xi}{r}+\tau, \lambda\right) d r^{\prime} d \xi,
$$

it follows that

$$
\mathrm{Op}_{M}^{\gamma}\left(\psi\left(r^{\prime} / r\right) f\right)(\lambda) u(r)=\mathrm{Op}_{r}(a)(\lambda) u(r)
$$

Formula (3.74) together with (3.82) shows that $a(r, \tau, \lambda)$ is as asserted.

Remark 13. Note that for $a(r, \rho, \lambda)=\tilde{a}(r \rho, \lambda)$ it follows that $f(i \rho, \eta)=\left(Q_{M}(\varphi) \tilde{a}\right)(i \rho, \lambda)$, i.e., $f$ is independent of $r$.

Theorem 11, Remark 12, and (12) can be generalised in many ways.

First of all we may admit symbols smoothly depending on several variables, for instance, the relations (3.70) and (12) generalise to

$$
\boldsymbol{Q}_{M}: C_{0}^{\infty}\left(\mathbb{R}_{+}\right) \times S_{(\mathrm{cl})}^{\mu}\left(\overline{\mathbb{R}}_{+} \times \Omega \times \mathbb{R}^{1+l}\right) \rightarrow S_{(\mathrm{cl})}^{\mu}\left(\overline{\mathbb{R}}_{+} \times \Omega \times \mathbb{C} \times \mathbb{R}^{l}\right)
$$

and

$$
\boldsymbol{Q}_{F}: C_{0}^{\infty}\left(\mathbb{R}_{+}\right) \times S_{(\mathrm{cl})}^{\mu}\left(\overline{\mathbb{R}}_{+} \times \Omega \times \Gamma_{\frac{1}{2}-\gamma} \times \mathbb{R}^{l}\right) \rightarrow S_{(\mathrm{cl})}^{\mu}\left(\overline{\mathbb{R}}_{+} \times \Omega \times C \times \mathbb{R}^{l}\right),
$$

respectively, for any open set $\Omega \subseteq \mathbb{R}^{q}$. A similar observation is true of Remark 12 and Theorem 12. 
Remark 14. Theorem 12 (i) can be regarded as a Mellin quantisation, Theorem 12 (ii) as an inverse Mellin quantisation.

For any $a(\xi, \lambda) \in L_{\mathrm{cl}}^{\mu}\left(X ; \mathbb{R}^{1+l}\right)$ and $\varphi(s) \in C_{0}^{\infty}\left(\mathbb{R}_{+}\right)$we form

$$
\left(Q_{M}(\varphi) a\right)(i \rho, \lambda):=\int_{\mathbb{R}} \int_{\mathbb{R}} e^{i(1-s) \rho} s^{-z} \varphi(s) a(\xi, \lambda) d s d \xi,
$$

$(z, \lambda) \in \mathbb{C} \times \mathbb{R}^{l}, z=1 / 2-\gamma+i \rho$. Moreover, for any $f(z, \lambda) \in L_{\mathrm{cl}}^{\mu}\left(X ; \Gamma_{1 / 2-\gamma} \times \mathbb{R}^{l}\right)$ and $\psi(s) \in C_{0}^{\infty}\left(\mathbb{R}_{+}\right)$ we set

$$
\left(Q_{F}(\psi) f\right)(\xi, \lambda):=\int_{\Gamma_{1 / 2-\gamma}} \int_{0}^{\infty} s^{z} e^{i(s-1) \xi} \psi(s) f(z, \lambda) \frac{d s}{s} d z
$$

$(\xi, \lambda) \in C \times \mathbb{R}^{l}$

\section{Theorem 13.}

(i) The formula (3.86) induces a separately continuous mapping

$$
Q_{M}: C_{0}^{\infty}\left(\mathbb{R}_{+}\right) \times L_{\mathrm{cl}}^{\mu}\left(X ; \mathbb{R}^{1+q}\right) \rightarrow M_{\mathscr{O}}^{\mu}\left(X ; \mathbb{R}^{q}\right)
$$

$(\varphi, a) \rightarrow Q_{M}(\varphi) a$. There are coefficients $c_{k j}(\varphi, \gamma)$ (only depending on $\left(\partial_{t}^{l} \varphi\right)(1), l \in \mathbb{N}$, and $\gamma \in \mathbb{R}$, but not on the symbol a) such that

$$
\left(Q_{M}(\varphi) a\right)(1 / 2-\gamma+i \rho, \eta) \sim \varphi(1) a(-\rho, \eta)+\sum_{k=1}^{\infty} \sum_{j=0}^{k} c_{k j}(\varphi, \gamma)(-\rho)^{j}\left(\partial_{\rho}^{k+j} a\right)(-\rho, \eta)
$$

$(\rho, \eta) \in \mathbb{R}^{1+q}$.

(ii) The formula (3.69) induces a separately continuous mapping

$$
\boldsymbol{Q}_{F}: C_{0}^{\infty}\left(\mathbb{R}_{+}\right) \times L_{\mathrm{cl}}^{\mu}\left(X ; \Gamma_{1 / 2-\gamma} \times \mathbb{R}^{q}\right) \rightarrow L_{\mathrm{cl}}^{\mu}\left(X ; C \times \mathbb{R}^{q}\right),
$$

$(\psi, f) \rightarrow Q_{F}(\psi) f$. There are coefficients $d_{k j}(\psi, \gamma)$ (only depending on $\left(\partial_{s}^{l} \psi\right)(1), l \in \mathbb{N}$, and $\gamma \in \mathbb{R}$, but not on the symbol $f$ ) such that

$$
\begin{aligned}
\left(\boldsymbol{Q}_{F}(\psi) f\right)(\xi, \lambda) \sim & \psi(1) f(1 / 2-\gamma-i \xi, \lambda) \\
& +\sum_{k=1}^{\infty} \sum_{j=0}^{k} d_{k j}(\psi, \gamma)(-i \xi)^{j}\left(\partial_{\xi}^{k+j} f\right)(1 / 2-\gamma-i \xi, \lambda),
\end{aligned}
$$

$(\xi, \lambda) \in \mathbb{R}^{1+l}$.

(iii) If $\varphi$ and $\psi$ are equal to 1 in a neighbourhood of 1 then we have

$$
\begin{aligned}
& \boldsymbol{Q}_{M}(\varphi) \boldsymbol{Q}_{F}(\psi) f=f \bmod L^{-\infty}\left(X ; \Gamma_{1 / 2-\gamma} \times \mathbb{R}^{l}\right), \\
& \boldsymbol{Q}_{F}(\psi) \boldsymbol{Q}_{M}(\varphi) a=a \bmod L^{-\infty}\left(X ; \mathbb{R}^{1+l}\right) .
\end{aligned}
$$

The following theorem is a Mellin quantisation result for edge-degenerate symbols. 
Theorem 14. Let $\varphi \in C_{0}^{\infty}\left(\mathbb{R}_{+}\right)$be equal to 1 near 1 and fix a weight $\gamma \in \mathbb{R}$. For $p(r, y, \rho, \eta):=$ $\tilde{p}(r, y, r \rho, r \eta), \tilde{p}(r, y, \tilde{\rho}, \tilde{\eta}) \in C^{\infty}\left(\overline{\mathbb{R}}_{+} \times \Omega, L_{\mathrm{cl}}^{\mu}\left(X ; \mathbb{R}_{\tilde{\rho}, \tilde{\eta}}^{1+q}\right)\right)$ and $h(r, y, z, \eta):=\tilde{h}(r, y, z, r \eta), \tilde{h}(r, y, z, \tilde{\eta}):=$ $\boldsymbol{Q}_{M}(\varphi) \tilde{p}(r, y, z, \tilde{\eta})$ belonging to $C^{\infty}\left(\overline{\mathbb{R}}_{+} \times \Omega, M_{\mathscr{O}}^{\mu}\left(X ; \mathbb{R}^{q}\right)\right)$, we have

$$
\mathrm{Op}_{r}(p)(y, \eta)-\mathrm{Op}_{M}^{\gamma}(h)(y, \eta)=\mathrm{Op}_{r}\left(\left(1-\varphi\left(r^{\prime} / r\right)\right) p\right)(y, \eta),
$$

where $\tilde{h}(r, y, z, \tilde{\eta}) \in C^{\infty}\left(\overline{\mathbb{R}}_{+} \times \Omega, M_{\mathscr{O}}^{\mu}\left(X ; \mathbb{R}^{q}\right)\right)$ and

$$
\mathrm{Op}_{r}\left(\left(1-\varphi\left(r^{\prime} / r\right)\right) p\right)(y, \eta) \in C^{\infty}\left(\Omega, L^{-\infty}\left(X^{\wedge} ; \mathbb{R}^{q}\right)\right) .
$$

Proof. The proof is a direct consequence of Theorem 12 (i).

Remark 15. Let $\tilde{h}(r, y, z, \tilde{\eta})$ and $\tilde{p}(r, y, \tilde{\rho}, \tilde{\eta})$ be as in Theorem 14 and set

$$
p_{0}(r, y, \rho, \eta):=\tilde{p}(0, y, r \rho, r \eta), h_{0}(r, y, z, \eta):=\tilde{h}(0, y, z, r \eta)
$$

Then we have $\mathrm{Op}_{r}\left(p_{0}\right)(y, \eta)-\mathrm{Op}_{M}^{\gamma}\left(h_{0}\right)(y, \eta)=\mathrm{Op}_{r}\left(\left(1-\varphi\left(r^{\prime} / r\right)\right) p_{0}\right)(y, \eta)$; the right hand side is again an element of $C^{\infty}\left(\Omega, L^{-\infty}\left(X^{\wedge} ; \mathbb{R}^{q}\right)\right)$.

Theorem 15. Let $\psi \in C_{0}^{\infty}\left(\mathbb{R}_{+}\right)$be equal to 1 near 1 and fix a weight $\gamma \in \mathbb{R}$. For $h(r, y, z, \eta):=$ $\tilde{h}(r, y, z, r \eta), \tilde{h}(r, y, z, \tilde{\eta}) \in C^{\infty}\left(\overline{\mathbb{R}}_{+} \times \Omega, M_{\mathscr{O}}^{\mu}\left(X ; \mathbb{R}_{\tilde{\eta}}^{q}\right)\right)$ and $p(r, y, \rho, \eta):=\tilde{p}(r, y, r \rho, r \eta), \tilde{p}(r, y, \tilde{\rho}, \tilde{\eta})=$ $\boldsymbol{Q}_{F}(\psi) \tilde{h}(r, y, \tilde{\rho}, \tilde{\eta})$ belonging to $C^{\infty}\left(\overline{\mathbb{R}}_{+} \times \Omega, L_{\mathrm{cl}}^{\mu}\left(X ; \mathbb{R}_{\tilde{\rho}, \tilde{\eta}}^{1+q}\right)\right)$, we have

$$
\mathrm{Op}_{M}^{\gamma}(h)(y, \eta)-\mathrm{Op}_{r}(p)(y, \eta)=\mathrm{Op}_{M}^{\gamma}\left(\left(1-\psi\left(r^{\prime} / r\right)\right) h\right)(y, \eta)
$$

where

$$
\mathrm{Op}_{M}^{\gamma}\left(\left(1-\psi\left(r^{\prime} / r\right)\right) h\right)(y, \eta) \in C^{\infty}\left(\Omega, L^{-\infty}\left(X^{\wedge} ; \mathbb{R}^{q}\right)\right)
$$

Proof. The proof is a direct consequence of Theorem 12 (ii).

Remark 16. Let $\tilde{h}(r, y, z, \tilde{\eta})$ and $\tilde{p}(r, y, \tilde{\rho}, \tilde{\eta})$ be as in Theorem 15 and

$$
p_{0}(r, y, \rho, \eta), h_{0}(r, y, z, \eta)
$$

as in Remark 15. Then we have $\mathrm{Op}_{r}\left(p_{0}\right)(y, \eta)-\mathrm{Op}_{M}^{\gamma}\left(h_{0}\right)(y, \eta)=\mathrm{Op}_{M}^{\gamma}\left(\left(1-\psi\left(r^{\prime} / r\right)\right) h_{0}\right)(y, \eta)$; the right hand side is again an element of $C^{\infty}\left(\Omega, L^{-\infty}\left(X^{\wedge} ; \mathbb{R}^{q}\right)\right)$.

\section{Boundary value problems are edge problems}

\subsection{Boundary value problems for differential operators}

Let us develope here some essential tools for understanding ellipticity of boundary value problems (BVPs). For instance, consider an embedded submanifold $M \subset \mathbb{R}^{n}$ with smooth boundary $\partial M=X$ and assume that $M$ is either compact or the half-space

$$
\overline{\mathbb{R}}_{+}^{n}:=\left\{x \in \mathbb{R}^{n}: x_{n} \geq 0\right\} .
$$


In order to see some examples, let $A$ be an elliptic operator in $\mathbb{R}^{n}$, first a differential operator of order $2 m, m \in \mathbb{N} \backslash\{0\}$. Among the best studied cases is the Laplacian $A=\Delta=\sum_{j=1}^{n} \frac{\partial^{2}}{\partial x_{j}^{2}}$ with Dirichlet boundary conditions. The Dirichlet problem

$$
\begin{gathered}
\Delta u=f \text { in int } M, \\
T_{0} u=g \text { on } X .
\end{gathered}
$$

for $T_{0} u:=\left.u\right|_{X}$ is elliptic with respect to a principal symbolic hierarchy

$$
\sigma(\mathscr{A}):=\left(\sigma_{\psi}(\mathscr{A}), \sigma_{\partial}(\mathscr{A})\right)
$$

for $\mathscr{A}=\left(\begin{array}{c}\Delta \\ T_{0}\end{array}\right)$, where $\sigma_{\psi}(\mathscr{A})(\xi)=\sigma_{\psi}(\Delta)(\xi)=-|\xi|^{2}, \xi \neq 0$ in this case is called the interior symbol and concerning notation in the following theorems, see [45, Definition 3.2.6].

$$
\sigma_{\partial}(\mathscr{A})(\eta):=\left(\begin{array}{c}
-|\eta|^{2}+\frac{\partial^{2}}{\partial x_{n}^{2}} \\
\gamma_{0}
\end{array}\right): H^{s}\left(\mathbb{R}_{+}\right) \rightarrow \begin{gathered}
H^{s-2}\left(\mathbb{R}_{+}\right) \\
\oplus
\end{gathered}
$$

the boundary symbol, $\gamma_{0} u:=u(0), \eta=\left(\xi_{1}, \ldots, \xi_{n-1}\right) \neq 0, s$ is assumed to be $>3 / 2$ ( the concrete choice will be unessential). The representations of $\sigma_{\psi}(\mathscr{A})$ and $\sigma_{\partial}(\mathscr{A})$ refer to representations in $\overline{\mathbb{R}}_{+}^{n}$, however, similarly as the invariance of $\sigma_{\psi}(\cdot)(x, \xi)$ as a function on $T^{*} M \backslash 0$ also the boundary symbol $\sigma_{\partial}(\cdot)(y, \eta)$ is invariantly defined as an operator function on $T^{*}(X) \backslash 0$.

In general, if $A=\sum_{|\alpha| \leq \mu} a_{\alpha}(x) D_{x}^{\alpha}$ is an elliptic differential operator of order $2 m$ then the boundary conditions are usually formulated in terms of differential operators $B_{j}, 0 \leq j \leq m-1$, of orders $0 \leq m_{j} \leq 2 m-1$, given in a collar neighborhood of the boundary $X$. The boundary conditions then have the form

$$
T u:=\left(\left.B_{j} u\right|_{\partial M}\right)_{j=0, \ldots, m-1}
$$

and the column matrix $\left(\begin{array}{l}A \\ T\end{array}\right)$ represents the boundary value problem

$$
A u=f \text { in int } M, \quad T u=g \text { on } X .
$$

As the pair of interior and boundary symbols we have in this case

$$
\sigma_{\psi}(A)(y, \xi)=\sum_{|\alpha|=\mu} a_{\alpha}(x) \xi^{\alpha}, \xi \neq 0,
$$

and

$$
\sigma_{\partial}(\mathscr{A})(y, \eta)=\left(\begin{array}{l}
\sigma_{\partial}(A) \\
\sigma_{\partial}(T)
\end{array}\right)(y, \eta)
$$


for

$$
\begin{aligned}
\sigma_{\partial}(A)(y, \eta) & :=\sigma_{\psi}(A)\left(y, 0, \eta, D_{x_{n}}\right) \\
\sigma_{\partial}(T)(y, \eta) u & :=\left(\left.\sigma_{\psi}\left(B_{j}\right)\left(y, 0, \eta, D_{x_{n}}\right) u\right|_{x_{n}=0}\right)_{j=0, \ldots, m-1}
\end{aligned}
$$

$\eta \neq 0$. Again the components of $\sigma(\mathscr{A})=\left(\sigma_{\psi}(A), \sigma_{\partial}(A)\right)$ are represented in coordinates in $\overline{\mathbb{R}}_{+}^{n}$, and the boundary symbol is operator-valued,

$$
\sigma_{\partial}(\mathscr{A})(y, \eta): H^{s}\left(\mathbb{R}_{+}\right) \rightarrow \quad \begin{gathered}
H^{s-\mu}\left(\mathbb{R}_{+}\right) \\
\mathbb{C}^{m}
\end{gathered}
$$

$s>\mu-1 / 2$.

Definition 5. The BVP $\mathscr{A}$ is said to be elliptic in the sense of Shapiro-Lopatinskij ellipticity of the boundary conditions $T$ with respect to the operator $A$ if $A$ itself is elliptic in the usual sense and (4.1) is a family of isomorphisms.

Elliptic BVPs in the sense of Definition 5 have been studied by numerous authors. Let us mention the classical papers of Lopatinskij [33], Agmon, Douglis and Nirenberg [1, 2], Seeley [52], Schechter [40], Solonnikov [56, 57], or the monograph of Lions and Magenes [32].

\subsection{Pseudo-differential boundary value problems with the transmission property}

In contrast to the case of ellipticity on an open smooth manifold and the comparatively simple structure of parametrices, cf. [13], it is not so easy to foresee the shape of a pseudodifferential algebra of boundary value problems, containing the parametrices of elliptic BVPs for differential operators. We outline here elements of the work of Boutet de Monvel [7]. More insight and useful generalisations have been developed in the monographs of Rempel and Schulze [35] and Grubb [23]. Later on, in connection with the development of the edge pseudo-differential calculus in [38] and [42] it turned out that BVPs are specific edge problems, see also the article of Schulze and Seiler [47]. This is also the motivation of the headline of the present Section 4. Later on, in subsequent parts of this exposition we will return many times to this aspect. The first novelty, as we saw in the preceding subsection, is the presence of a second principal symbolic component. Besides the interior symbol $\sigma_{\psi}(\cdot)$, we have here the boundary symbol $\sigma_{\partial}(\cdot)$ which determines the ellipticity of the respective BVPs. Similar phenomena occur in ellipticity of BVPs on a manifold with edge or higher singularities. Then, according to the higher stratification of the underlying singular manifold, we have a corresponding larger number of principal symbolic components. If $M$ is a manifold with smooth boundary the stratification consists of

$$
\text { int } M \quad \text { and } \quad \partial M=X \text {, }
$$


The strata are open smooth manifolds of different dimensions, and we have a disjoint union

$$
M=\operatorname{int} M \cup \partial M
$$

Let $\tilde{M}$ be an open smooth manifold, and let $M \hookrightarrow \tilde{M}$ be an embedding as a smooth submanifold with boundary. Then it makes sense to consider an $\tilde{A} \in L_{\mathrm{cl}}^{\mu}(\tilde{M})$ with its interior principal $\operatorname{symbol} \sigma_{\psi}(\tilde{A}) \in C^{\infty}\left(T^{*} \tilde{M} \backslash 0\right)$,

$$
\sigma_{\psi}(A):=\left.\sigma_{\psi}(\tilde{A})\right|_{T^{*}(\operatorname{int} M) \backslash 0} \text { for } A:=\left.\tilde{A}\right|_{\operatorname{int} M},
$$

and to interpret $\partial M \subset \tilde{M}$ as an additional input which generates a second principal symbolic component $\sigma_{\partial}(A)$. Locally near a point $x \in \partial M$ we can identify $\tilde{M}$ with $\mathbb{R}^{n}, \partial M$ with $\mathbb{R}^{n-1}$, and $M$ with $\overline{\mathbb{R}}_{+}^{n}$. Then, as in Subsection 4.1 , for a differential operator $A=\sum_{|\alpha| \leq \mu} a_{\alpha}(x) D_{x}^{\alpha}$ we set

$$
\begin{gathered}
\sigma_{\psi}(A)(x, \xi)=\sum_{|\alpha|=\mu} a_{\alpha}(x) \xi^{\alpha}, \\
\sigma_{\partial}(A)(y, \eta):=\sigma_{\psi}(A)\left(y, 0, \eta, D_{t}\right): H^{s}\left(\mathbb{R}_{+}\right) \rightarrow H^{s-\mu}\left(\mathbb{R}_{+}\right),
\end{gathered}
$$

for $x=(y, t) \in \mathbb{R}_{+}^{n}, y \in \mathbb{R}^{n-1}, t \in \mathbb{R}_{+}$. While the interior symbol is (positively) homogeneous in the standard sense, namely,

$$
\sigma_{\psi}(A)(x, \delta \xi)=\delta^{\mu} \sigma_{\psi}(A)(x, \xi)
$$

$\delta \in \mathbb{R}_{+}$, the boundary symbol is twisted homogeneous in the sense

$$
\sigma_{\partial}(A)(y, \delta \eta)=\delta^{\mu} \kappa_{\delta} \sigma_{\partial}(A)(y, \eta) \kappa_{\delta}^{-1},
$$

$\delta \in \mathbb{R}_{+}$. Here $\left(\kappa_{\delta} u\right)(t):=\delta^{1 / 2} u(\delta t)$ determines a group action on the spaces $H^{s}\left(\mathbb{R}_{+}\right), s \in \mathbb{R}$. As we see the boundary symbol (4.3) takes values in differential operators on $\mathbb{R}_{+}$.

Remark 17. Let $A$ be elliptic, i.e., $\sigma_{\psi}(A)(x, \xi) \neq 0$ for $\xi \neq 0$. Then the operators (4.3) are elliptic on $\mathbb{R}_{+}$for $\eta \neq 0$, where

$$
\sigma_{\psi}(A)^{-1}\left(y, 0, \eta, D_{t}\right)=: \sigma_{\partial}(A)^{(-1)}(y, \eta)
$$

is a pseudo-differential parametrix over $\mathbb{R}_{+}$.

In the case of a pseudo-differential operator on $\mathbb{R}_{+}$it is not a priori clear how we define an action in the opposite direction of (4.3), namely, $H^{s-\mu}\left(\mathbb{R}_{+}\right) \rightarrow H^{s}\left(\mathbb{R}_{+}\right)$. We adopt here the truncation operator convention. To this end we observe that

$$
a(y, t, \eta, \tau):=\sum_{|\alpha| \leq \mu} a_{\alpha}(y, t)(\eta, \tau)^{\alpha}
$$


belongs to $S_{\mathrm{cl}}^{\mu}\left(\mathbb{R}_{t} \times \mathbb{R}_{\tau}\right)$ for every fixed $(y, \eta)$. For any such symbol we define

$$
\operatorname{op}^{+}(a)(y, \eta):=\mathrm{r}^{+} \mathrm{op}(a)(y, \eta) \mathrm{e}^{+}: C_{0}^{\infty}\left(\mathbb{R}_{+}\right) \rightarrow C^{\infty}\left(\mathbb{R}_{+}\right)
$$

with $\mathrm{e}^{+}$being the operator of extension by zero to the opposite half-line, i.e.,

$$
\mathrm{e}^{+} u=\left\{\begin{array}{l}
u \text { for } t>0 \\
0 \text { for } t \leq 0
\end{array},\right.
$$

and $\mathrm{r}^{+} f:=\left.f\right|_{\mathbb{R}_{+}}$, the restriction of distributions to $\mathbb{R}_{+}$. Moreover,

$$
\operatorname{op}(a)(y, \eta) v(t):=\iint e^{i\left(t-t^{\prime}\right) \tau} a(y, t, \eta, \tau) v\left(t^{\prime}\right) d t^{\prime} d \tau
$$

for $d \tau=(2 \pi)^{-1} d \tau$. This notation is valid for arbitrary symbols $a(y, t, \eta, \tau) \in S_{\mathrm{cl}}^{\mu}\left(\mathbb{R}^{n-1} \times \mathbb{R} \times\right.$ $\mathbb{R}_{\eta, \tau}^{n}$ ), and we obtain a family of continuous maps (4.6). The same is true of $a_{0}^{-1}(y, \eta, \tau):=$ $a^{-1}(y, 0, \eta, \tau) \in S_{\mathrm{cl}}^{-\mu}\left(\mathbb{R}_{\tau}\right)$ for every fixed $y$ and $\eta \neq 0$, as it appears in (4.4) for (4.5), coming from an elliptic operator $A$. However these symbols in $\tau$ are very specific. We have in this case for $\eta \neq 0$

$$
a^{-1}(y, 0, \eta, \tau) \neq 0
$$

for all $\tau \in \mathbb{R}$. Moreover, we see in this case for $a_{0}(y, \eta, \tau):=a(y, 0, \eta, \tau)$

$$
\operatorname{op}^{+}\left(a_{0}\right)(y, \eta) \operatorname{op}^{+}\left(a_{0}^{-1}\right)(y, \eta)=\operatorname{op}^{+}\left(a_{0} a_{0}^{-1}\right)(y, \eta)=1
$$

since

$$
\operatorname{op}^{+}\left(a_{0}\right) \operatorname{op}^{+}\left(a_{0}^{-1}\right)=\mathrm{r}^{+} \mathrm{op}\left(a_{0}\right) \mathrm{e}^{+} \mathrm{r}^{+} \mathrm{op}\left(a_{0}^{-1}\right) \mathrm{e}^{+}=\mathrm{r}^{+} \mathrm{op}\left(a_{0}\right) \mathrm{op}\left(a_{0}^{-1}\right) \mathrm{e}^{+}
$$

which is a consequence of the locality of op $\left(a_{0}\right)$ as a differential operator, i.e., $\operatorname{suppop}\left(a_{0}\right) f \subseteq$ $\operatorname{supp} f$, applied to $f:=\mathrm{e}^{+} \mathrm{r}^{+} \mathrm{op}\left(a_{0}^{-1}\right) \mathrm{e}^{+} u$. This conclusion is valid for any $u \in H^{s-\mu}\left(\mathbb{R}_{+}\right)$for $s-\mu>-1 / 2$, since then $\mathrm{e}^{+} u \in \mathscr{S}^{\prime}(\mathbb{R})$ and $\operatorname{supp} \mathrm{e}^{+} \mathrm{r}^{+} \operatorname{op}\left(a_{0}^{-1}\right) \mathrm{e}^{+} u \subseteq \overline{\mathbb{R}}_{+}$with op $\left(a_{0}^{-1}\right) \mathrm{e}^{+} u$ being applied in the distributional sense, cf. [45] and [13].

Definition 6. An $a(t, \tau) \in S_{\mathrm{cl}}^{\mu}(\mathbb{R} \times \mathbb{R}), \mu \in \mathbb{Z}$, is said to have the transmission property at $t=0$ if the coefficients $a_{j}^{ \pm}(t) \in C^{\infty}(\mathbb{R})$ in asymptotic expansions

$$
a(t, \tau) \sim \sum_{j=0}^{\infty} a_{j}^{ \pm}(t)(i \tau)^{\mu-j} \quad \text { as } \tau \rightarrow \pm \infty
$$

( $i=\sqrt{-1}$ is used for technical reasons) satisfy the conditions

$$
D_{t}^{k} a_{j}^{+}(0)=D_{t}^{k} a_{j}^{-}(0) \quad \text { for all } j, k \in \mathbb{N} .
$$

In a similar manner we define the transmission property of a symbol $a(t, \tau) \in S_{\mathrm{cl}}^{\mu}\left(\overline{\mathbb{R}}_{+} \times \mathbb{R}\right)$. 
In particular, if $a(\tau) \in S_{\mathrm{cl}}^{\mu}(\mathbb{R})$ is a symbol with constant coefficients (i.e., independent of $t$ ) then the transmission property means

$$
a_{j}^{+}=a_{j}^{-} \text {for all } j \in \mathbb{N} \text {. }
$$

Remark 18. Every $a(t, \tau) \in S_{\mathrm{cl}}^{\mu}(\mathbb{R} \times \mathbb{R})$ has an asymptotic expansion for coefficients $a_{j}^{ \pm}(t) \in$ $C^{\infty}\left(\mathbb{R}_{+}\right)$that are completely independent, and Theorem 16 shows that for every choice of constants $a_{j}^{+}$and $a_{j}^{-}, j \in \mathbb{N}$, there exists a symbol with thee coefficients. So we see that generically the transmission property is violated. Both the cases with and without the transmission property are important for several applications.

Theorem 16. Let $a(\tau) \in S_{\mathrm{cl}}^{\mu}(\mathbb{R})$ have the transmission property. Then the truncated operator $\mathrm{op}^{+}($a) induces continuous maps

$$
\operatorname{op}^{+}(a): H^{s}\left(\mathbb{R}_{+}\right) \rightarrow H^{s-\mu}\left(\mathbb{R}_{+}\right)
$$

for all $s \in \mathbb{R}, s>-1 / 2$.

Let us now return to (4.4). We then obtain a continuous operator

$$
\sigma_{\partial}(A)^{-1}(y, \eta): H^{s-\mu}\left(\mathbb{R}_{+}\right) \rightarrow H^{s}\left(\mathbb{R}_{+}\right)
$$

for every $s \in \mathbb{R}, s-\mu>-1 / 2$. Moreover (4.7) tells us that (4.8) is a right inverse inverse of $\sigma_{\partial}(A)(y, \eta)$. Thus $\sigma_{\partial}(A)(y, \eta): H^{s}\left(\mathbb{R}_{+}\right) \rightarrow H^{s-\mu}\left(\mathbb{R}_{+}\right)$is a surjective operator for $\eta \neq 0$.

In higher dimensions for symbols over $\Omega \times \mathbb{R}, \Omega \subseteq \mathbb{R}^{q}$ open, the transmission property is defined as follows.

Definition 7. A symbol $a(y, t, \eta, \tau) \in S_{\mathrm{cl}}^{\mu}\left(\Omega \times \mathbb{R} \times \mathbb{R}_{\eta, \tau}^{n}\right)$ for $n=q+1, \mu \in \mathbb{Z}$, is said to have the transmission property at $t=0$ if it satisfies the conditions

$$
D_{t}^{k} D_{\xi}^{\alpha}\left\{a_{(\mu-j)}(y, 0,0,1)-(-1)^{\mu-j-|\alpha|} a_{(\mu-j)}(y, 0,0,-1)\right\}=0
$$

for all $y \in \Omega, \xi=(\eta, \tau)$, for all $k \in \mathbb{N}, \alpha \in \mathbb{N}^{q}, j \in \mathbb{N}$ (recall that $a_{(\mu-j)}$ denotes the homogeneous component of $a$ of order $\mu-j$ ). Let

$$
S_{\mathrm{tr}}^{\mu}\left(\Omega \times \mathbb{R} \times \mathbb{R}^{n}\right)
$$

be the space of all symbols of that kind. Moreover, set

$$
S_{\mathrm{tr}}^{\mu}\left(\Omega \times \overline{\mathbb{R}}_{ \pm} \times \mathbb{R}^{n}\right):=\left\{\left.a\right|_{\Omega \times \overline{\mathbb{R}}_{ \pm} \times \mathbb{R}^{n}}: a \in S_{\mathrm{tr}}^{\mu}\left(\Omega \times \mathbb{R} \times \mathbb{R}^{n}\right)\right\}
$$


Let us now apply spaces of operator-valued symbols

$$
S^{\mu}\left(\Omega \times \mathbb{R}^{q} ; H, \widetilde{H}\right),
$$

cf. [13, Definition 3.5], here for the case $H:=H^{s}\left(\mathbb{R}_{+}\right), \widetilde{H}:=H^{s-\mu}\left(\mathbb{R}_{+}\right)$, or the Fréchet space $\mathscr{S}\left(\overline{\mathbb{R}}_{+}\right)$in place of $H$ or $\tilde{H}$, endowed with the group action $\kappa=\left\{\kappa_{\delta}\right\}_{\delta \in \mathbb{R}_{+}}$defined by $\left(\kappa_{\delta} u\right)(t):=$ $\delta^{1 / 2} u(\delta t)$.

Proposition 5. Let $\Omega \subset \mathbb{R}^{q}$ be an open set, and assume that $a(y, t, \eta, \tau) \in S_{\mathrm{tr}}^{\mu}\left(\Omega \times \overline{\mathbb{R}}_{+} \times \mathbb{R}^{n}\right)$ is independent of $t$ for $t>c$ for some constant $c>0$. Then we have

$$
\operatorname{op}^{+}(a)(y, \eta) \in S^{\mu}\left(\Omega \times \mathbb{R}^{n-1} ; H^{s}\left(\mathbb{R}_{+}\right), H^{s-\mu}\left(\mathbb{R}_{+}\right)\right)
$$

for every real $s>-1 / 2$, and

$$
\operatorname{op}^{+}(a)(y, \eta) \in S^{\mu}\left(\Omega \times \mathbb{R}^{n-1} ; \mathscr{S}\left(\overline{\mathbb{R}}_{+}\right), \mathscr{S}\left(\overline{\mathbb{R}}_{+}\right)\right) .
$$

The operator-valued symbol $\mathrm{op}^{+}(a)$ is classical when a is independent of $t$.

The technicalities to prove Proposition 5 may be found in [45].

On a manifold $M$ with smooth boundary we have the double $2 M$, obtained by gluing together two copies of $M$ along the common boundary. Consider $L_{\mathrm{cl}}^{\mu}(2 M)$, the space of classical pseudo-differential operators on $2 M$ of order $\mu \in \mathbb{R}$. An $\tilde{A} \in L_{\mathrm{cl}}^{\mu}(2 M)$ for $\mu \in \mathbb{Z}$ is said to have the transmission property at $\partial M$ if modulo an operator in $L^{-\infty}(2 M)$ it has close to the boundary in local coordinates $(y, t) \in \Omega \times \mathbb{R}$ the form

$$
\tilde{A}=\operatorname{Op}(\tilde{a})
$$

for a symbol $\tilde{a}(y, t, \eta, \tau) \in S_{\mathrm{tr}}^{\mu}\left(\Omega \times \mathbb{R} \times \mathbb{R}^{n}\right)$. The space of all elements of $L_{\mathrm{cl}}^{\mu}(2 M)$ with the transmission property at $\partial M$ will be denoted by $L_{\mathrm{tr}}^{\mu}(2 M)$, and we set

$$
L_{\mathrm{tr}}^{\mu}(M):=\left\{A=\mathrm{r}^{+} \tilde{A} \mathrm{e}^{+}: \tilde{A} \in L_{\mathrm{tr}}^{\mu}(2 M)\right\}
$$

Here, similarly as before, $\mathrm{e}^{+}$means extension from int $M$ by zero to $2 M$, and $\mathrm{r}^{+}$restriction of distributions from $2 M$ to int $M$.

From the definition it follows that modulo a smoothing operator (i.e., an operator with kernel in $\left.C^{\infty}(M \times M)\right)$ close to the boundary any $A \in L_{\mathrm{tr}}^{\mu}(M)$ can be written as $\mathrm{Op}_{y}\left(\mathrm{op}^{+}(a)\right)$ for an $a \in S_{\mathrm{tr}}^{\mu}\left(\Omega \times \mathbb{R} \times \mathbb{R}_{y, t}^{n}\right)$. This gives us the following

Proposition 6. Let $M$ be a compact manifold with smooth boundary. Then every $A \in L_{\mathrm{tr}}^{\mu}(M)$ induces continuous operators

$$
A: H^{s}(\text { int } M) \rightarrow H^{s-\mu}(\operatorname{int} M)
$$

for every real $s>-\frac{1}{2}$, and

$$
A: C^{\infty}(M) \rightarrow C^{\infty}(M) .
$$


If $M$ is not compact, we have a similar result referring to comp/loc-spaces, or to operators $C_{0}^{\infty}(M) \rightarrow C^{\infty}(M)$.

For a pseudo-differential calculus of boundary value problems, here with the transmission property, it is essential to compose operators within the structure including a control of symbols. Similarly as for differential operators, cf. (4.2), (4.3), also $A \in L_{\mathrm{tr}}^{\mu}(M)$ has a principal symbolic structure

$$
\sigma(A):=\left(\sigma_{\psi}(A), \sigma_{\partial}(A)\right)
$$

consisting of interior and boundary symbol, where $\sigma_{\psi}(A)(x, \xi)$ is the standard homogeneous principal symbol of $A$ of order $\mu$, while $\sigma_{\partial}(A)(y, \eta)$ is the boundary symbol, is defined as the operator function

$$
\sigma_{\partial}(A)(y, \eta):=\operatorname{op}^{+}\left(\left.a_{(\mu)}\right|_{t=0}\right)(y, \eta): H^{s}\left(\mathbb{R}_{+}\right) \rightarrow H^{s-\mu}\left(\mathbb{R}_{+}\right)
$$

for $\eta \neq 0$. It turns out that compositions generate remainders, so-called Green operators. The notation comes from Green's function in solution formulas to elliptic boundary value problems. More precisely, if we construct the solution of an elliptic BVP $\mathscr{A}$ by computing the inverse $\mathscr{A}^{-1}$ which is a special parametrix of $\mathscr{A}$, then Green operators appear in a natural way. We saw such Green contributions already in [13, formula (4.4)], namely, $G:=\mathrm{Op}_{y}(g)$ for the operator function $g(\eta)$, here with constant coefficients. In general, Green symbols $g(y, \eta)$ are defined as follows.

\section{Definition 8.}

(i) An operator-valued symbol

$$
g(y, \eta) \in S_{\mathrm{cl}}^{\mu}\left(\Omega \times \mathbb{R}^{q} ; L^{2}\left(\mathbb{R}_{+}\right), L^{2}\left(\mathbb{R}_{+}\right)\right)
$$

is called a Green symbol of order $\mu \in \mathbb{R}$ and type 0 if $g(y, \eta)$ and its pointwise $L^{2}\left(\mathbb{R}_{+}\right)$adjoint $g^{*}(y, \eta)$ constitute elements

$$
g(y, \eta), g^{*}(y, \eta) \in S_{\mathrm{cl}}^{\mu}\left(\Omega \times \mathbb{R}^{q} ; L^{2}\left(\mathbb{R}_{+}\right), \mathscr{S}\left(\overline{\mathbb{R}}_{+}\right)\right)
$$

$\mathscr{S}\left(\overline{\mathbb{R}}_{+}\right)=\left.\mathscr{S}(\mathbb{R})\right|_{\mathbb{R}_{+}}$.

(ii) An operator-valued symbol

$$
g(y, \eta) \in S_{\mathrm{cl}}^{\mu}\left(\Omega \times \mathbb{R}^{q} ; H^{s}\left(\mathbb{R}_{+}\right), L^{2}\left(\mathbb{R}_{+}\right)\right)
$$

for $d \in \mathbb{N}, s-d>-1 / 2$, is called a Green symbol of order $\mu \in \mathbb{R}$ and type $d$ if there are Green symbols $g_{j}(y, \eta)$ of order $\mu-j$ and type $0, j=0, \ldots, d$, such that

$$
g(y, \eta)=\sum_{j=0}^{d} g_{j}(y, \eta) \frac{d^{j}}{d t^{j}} .
$$


Remark 19. Observe that a $g \in \mathscr{L}\left(L^{2}\left(\mathbb{R}_{+}\right)\right)$which induces continuous operators $g, g^{*}: L^{2}\left(\mathbb{R}_{+}\right)$ $\rightarrow \mathscr{S}\left(\overline{\mathbb{R}}_{+}\right)$has a kernel $k_{g}\left(t, t^{\prime}\right) \in \mathscr{S}\left(\overline{\mathbb{R}}_{+}\right) \hat{\otimes}_{\pi} \mathscr{S}\left(\overline{\mathbb{R}}_{+}\right)=\left.\mathscr{S}(\mathbb{R} \times \mathbb{R})\right|_{\overline{\mathbb{R}}_{+} \times \overline{\mathbb{R}}_{+}}$, i.e., $g u(t)=\int_{0}^{\infty} k_{g}\left(t, t^{\prime}\right)$ $u\left(t^{\prime}\right) d t^{\prime}$ for all $u \in L^{2}\left(\mathbb{R}_{+}\right)$. This implies that a $\mathrm{g}$ as in Definition 8 (i) defines a symbol (4.13) for every $s>-1 / 2$.

Let $\mathscr{R}_{G}^{\mu, d}\left(\Omega \times \mathbb{R}^{q}\right)$ denote the space of all Green symbols of order $\mu$ and type $d$, and form $G:=\mathrm{Op}_{y}(g)$, called a (local) Green operator with such a symbol. On a manifold $M$ with smooth boundary we define the space $B_{G}^{-\infty, d}(M)$ as the set of all operators of the form

$$
\sum_{j=0}^{d} C_{j} \frac{d^{j}}{d t^{j}} \quad \text { for arbitrary } \quad C_{j} \quad \text { with kernels in } \quad C^{\infty}(M \times M) .
$$

Here $d / d t$ means the derivative in normal direction to $\partial M$. Now the space of Green operators $B_{G}^{\mu, d}(M)$ on $M$ of order $\mu$ and type $d$ is the set of all $G \in L^{-\infty}$ (int $M$ ) that are up to a $C \in B_{G}^{-\infty, d}(M)$ locally near the boundary of the form $\mathrm{Op}_{y}(g)$ for some $g \in \mathscr{R}_{G}^{\mu, d}\left(\Omega \times \mathbb{R}^{q}\right)$. Green operators $G$ can be constructed from local terms by using charts on $M$ near $\partial M$, a subordinate partition of unity, etc. We set $\sigma_{\psi}(G)=0$ and

$$
\sigma_{\partial}(G)(y, \eta):=g_{(\mu)}(y, \eta)
$$

where $g_{(\mu)}(y, \eta)$ is the (twisted homogeneous) principal symbol of $g(y, \eta)$, as a classical operatorvalued symbol of order $\mu$.

Definition 9. The space $B^{\mu, d}(M)$ is defined as the set of all $A+G$ for any $A \in L_{\mathrm{tr}}^{\mu}(M)$ and $G \in B_{G}^{\mu, d}(M)$. We set

$$
\sigma_{\psi}(A+G):=\sigma_{\psi}(A), \quad \sigma_{\partial}(A+G):=\sigma_{\partial}(A)+\sigma_{\partial}(G) .
$$

The operators in $B^{\mu, d}(M)$ constitute an ingredient of the space of BVPs on $M$ with the transmission property at $\partial M$.

Theorem 17. Let $A \in B^{\mu, d}(M), B \in B^{v, e}(M)$; then we have $A B \in B^{\mu+v, f}(M)$ for $f=\max \{v+d, e\}$, and

$$
\sigma_{\psi}(A B)=\sigma_{\psi}(A) \sigma_{\psi}(B), \quad \sigma_{\partial}(A B)=\sigma_{\partial}(A) \sigma_{\partial}(B) .
$$

If $A$ or $B$ is a Green operator so is $A B$.

Ellipticity of an element in $A \in B^{\mu, d}(M)$ is determined not only by $\sigma_{\psi}(A)$ but also $\sigma_{\partial}(A)$. An operator $A$ is called $\sigma_{\psi}$-elliptic if $\sigma_{\psi}(A) \neq 0$ on $T^{*} M \backslash 0$ (with 0 indicationg the zero section) The boundary symbol $\sigma_{\partial}(A)$ is operator-valued, it defines a family of continuous operators

$$
\sigma_{\partial}(A)(y, \eta): H^{s}\left(\mathbb{R}_{+}\right) \rightarrow H^{s-\mu}\left(\mathbb{R}_{+}\right)
$$


for any fixed $s>d-1 / 2$, and $(y, \eta) \in T^{*}(\partial M) \backslash 0$, and an analogue of the invertibility of $\sigma_{\psi}(A)$ for boundary symbols would be the invertibility of (4.15). However, the invertibility of (4.15) under the condition of $\sigma_{\psi}$-ellipticity of $A$ is only true in exceptional cases. In general we have the following

Lemma 5. Let $A \in B^{\mu, d}(M)$ be $\sigma_{\psi}$-elliptic. Then (4.15) is a family of Fredholm operators for all $s>\max \{\mu, d\}>-1 / 2$, and

$$
\operatorname{dim} \operatorname{ker} \sigma_{\partial}(A)(y, \eta), \operatorname{dim} \operatorname{coker} \sigma_{\partial}(A)(y, \eta)
$$

are independent of $s$.

The way of organising a bijective boundary symbol in connection with $A \in B^{\mu, d}(M)$ is to pass to a $2 \times 2$ operator block matrix

$$
\mathscr{A}=\left(\begin{array}{cc}
A+G & K \\
T & Q
\end{array}\right)
$$

with a Green operator $G$, a so-called trace operator $T$, a potential operator $K$, and a pseudodifferential operator $Q$ on $\partial M$. The structure of those operators is close to the one of Green operators. Therefore, we simply enlarge Definition 8 as follows.

Definition 10. An operator-valued symbol

$$
g(y, \eta):=\left(g_{l, m}(y, \eta)\right)_{l, m=1,2} \in S_{\mathrm{cl}}^{\mu}\left(\Omega \times \mathbb{R}^{q} ; H^{s}\left(\mathbb{R}_{+}\right) \oplus \mathbb{C}^{j_{-}}, L^{2}\left(\mathbb{R}_{+}\right) \oplus \mathbb{C}^{j_{+}}\right)
$$

for some $j_{-}, j_{+} \in \mathbb{N}$ and $d \in \mathbb{N}, s-d>-\frac{1}{2}$, is called a Green symbol of order $\mu \in \mathbb{R}$ and type $d$ if $g_{1,1}(y, \eta) \in \mathscr{R}_{G}^{\mu, d}\left(\Omega \times \mathbb{R}^{q}\right)$, cf. Definition 8, $g_{1,2}(y, \eta) \in S_{\mathrm{cl}}^{\mu}\left(\Omega \times \mathbb{R}^{q} ; \mathbb{C}^{j_{-}}, \mathscr{S}\left(\overline{\mathbb{R}}_{+}\right)\right), g_{2,1}(y, \eta) \in$ $S_{\mathrm{cl}}^{\mu}\left(\Omega \times \mathbb{R}^{q} ; H^{s}\left(\mathbb{R}_{+}\right), \mathbb{C}^{j_{+}}\right)$, where the $i$-th component of $g_{2,1}(y, \eta)$ is of the form

$$
g_{2,1}(y, \eta)_{i}=\sum_{b=0}^{d} t_{i, b}(y, \eta) \frac{d^{b}}{d t^{b}}
$$

for symbols $t_{i, b}(y, \eta):=k_{i, b}^{*}(y, \eta), k_{i, b}(y, \eta) \in S_{\mathrm{cl}}^{\mu}\left(\Omega \times \mathbb{R}^{q} ; \mathbb{C}, \mathscr{S}\left(\overline{\mathbb{R}}_{+}\right)\right), i=0, \ldots, j_{+}$, while $g_{2,2}(y, \eta) \in$ $S_{\mathrm{cl}}^{\mu}\left(\Omega \times \mathbb{R}^{q} ; \mathbb{C}^{j_{-}}, \mathbb{C}^{j_{+}}\right)$

The group actions in the spaces on the right of (4.17) are defined as $\kappa_{\delta} \oplus \mathrm{id}_{\mathbb{C}^{j^{ \pm}}}$for $\left(\kappa_{\delta} u\right)(\delta t)$ $=\delta^{1 / 2} u(\delta t), \delta \in \mathbb{R}_{+}$.

On a manifold $M$ with smooth boundary we define the space $B_{\text {potential }}^{-\infty}(M)$ as the set of all operators with kernel in $C^{\infty}(M \times \partial M)$. Moreover, we define the space $B_{\text {potential }}^{\mu}(M)$ of potential operators $K: C^{\infty}(\partial M) \rightarrow C^{\infty}(M)$ that are modulo an element of $B_{\text {potential }}^{-\infty}(M)$ locally near the boundary of the form $\mathrm{Op}_{y}\left(g_{1,2}\right)$ for some $g_{1,2}$ as in Definition 10 (for $j_{-}=0$ ). 
Moreover, we define the space $B_{\text {trace }}^{-\infty, d}(M)$ as the set of all operators of the form

$$
\sum_{j=0}^{d} D_{j} \frac{d^{j}}{d t^{j}} \quad \text { for arbitrary } \quad D_{j} \quad \text { with kernels in } \quad C^{\infty}(\partial M \times M) .
$$

Here $d / d t$ means again the derivative in normal direction to $\partial M$. Now the space of trace operators $B_{\text {trace }}^{\mu, d}(M)$ on $M$ of order $\mu$ and type $d$ is the set of all operators $T: C^{\infty}(M) \rightarrow C^{\infty}(\partial M)$ that are up to an element of $B_{\text {trace }}^{-\infty, d}(M)$ locally near the boundary of the form $\operatorname{Op}_{y}\left(g_{2,1}\right)$ for some $g_{2,1}$ as in Definition 10 (for $j_{+}=0$ ).

Let $B_{G}^{\mu, d}\left(M ; j_{-}, j_{+}\right)$be the space of all operators

$$
\left(\begin{array}{ll}
G & K \\
T & Q
\end{array}\right)
$$

where $G \in B_{G}^{\mu, d}(M)$, moreover, $K$ is a row vector of potential operators and $T$ a column vector of trace perators, with $j_{-}$and $j_{+}$components, respectively, and $Q$ is a $j_{+} \times j_{-}$-matrix of operators in $L_{\mathrm{cl}}^{\mu}(\partial M)$.

Definition 11. Let $B^{\mu, d}\left(M ; j_{-}, j_{+}\right)$denote the set of all operator block matrices $\mathscr{A}$ of the form (4.16) for arbitrary $A \in B^{\mu, d}(M)$, where the remaining entries are as in (4.18).

Similarly as for upper left corners we have a pair $\sigma(\mathscr{A})=\left(\sigma_{\psi}(\mathscr{A}), \sigma_{\partial}(\mathscr{A})\right)$ of principal symbols, where $\sigma_{\psi}(\mathscr{A})(x, \xi):=\sigma_{\psi}(A)(x, \xi)$, is the interior symbol, while the boundary symbol $\sigma_{\partial}(\mathscr{A})(y, \eta)$ is a family of block matrix operators

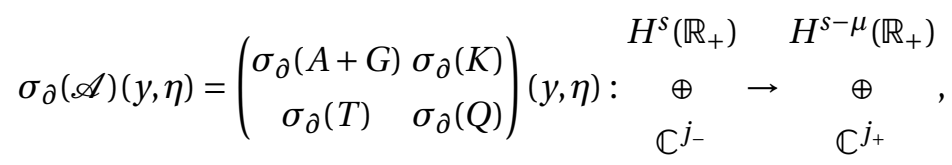

$s-d>-1 / 2$.

For operators in $B^{\mu, d}\left(M ; j_{-}, j_{+}\right)$we have a similar composition result as Theorem 17 , where we assume that rows and columns in the middle fit together.

Definition 12. An operator $\mathscr{A} \in B^{\mu, d}\left(M ; j_{-}, j_{+}\right)$is called $\left(\sigma_{\psi}, \sigma_{\partial}\right)$-elliptic, if its upper left corner is $\sigma_{\psi}$-elliptic and if (4.19) is a family of isomorphisms for all $(y, \eta) \in T^{*}(\partial M) \backslash 0$, and $s>\max \{d, \mu\}-\frac{1}{2}$.

Theorem 18. For an operator $\mathscr{A} \in B^{\mu, d}\left(M ; j_{-}, j_{+}\right)$the following properties are equivalent:

(i) $\mathscr{A}$ is $\left(\sigma_{\psi}, \sigma_{\partial}\right)$-elliptic, 
(ii) the operator $\mathscr{A}$ induces a Fredholm operator

$$
\begin{aligned}
& H^{s}(M) \quad H^{s-\mu}(M) \\
& \mathscr{A}: \underset{H^{s}\left(\partial M, \mathbb{C}^{j_{-}}\right)}{\rightarrow} \quad \begin{array}{l}
\oplus \\
H^{s-\mu}\left(\partial M, \mathbb{C}^{j_{+}}\right)
\end{array}
\end{aligned}
$$

for every $s>\max \{d, \mu\}-1 / 2$.

Theorem 19. $A\left(\sigma_{\psi}, \sigma_{\partial}\right)$-elliptic operator $\mathscr{A} \in B^{\mu, d}\left(M ; j_{-}, j_{+}\right)$has a parametrix $\mathscr{R} \in B^{-\mu, d_{\mathrm{R}}}\left(M ; j_{+}, j_{-}\right)$in the sense that

$$
\mathscr{R} \mathscr{A}-\mathscr{I} \in B_{G}^{-\infty, d_{\mathrm{L}}}\left(M ; j_{-}, j_{-}\right), \quad \mathscr{A} \mathscr{R}-\mathscr{I} \in B_{G}^{-\infty, d_{\mathrm{R}}}\left(M ; j_{+}, j_{+}\right)
$$

for $d_{\mathrm{L}}=\max \{d, \mu\}, d_{\mathrm{R}}=\max \{d-\mu, 0\}$.

\section{Examples and additional comments on boundary value problems}

\subsection{Stratifications and higher symbols for the Laplacian}

A good example of boundary value problems is the mixed elliptic boundary value problems for elliptic differential operators in a domain $M$ in $\mathbb{R}^{n}$ with smooth boundary $\partial M=X$. Here we focus on the Zaremba problem for the Laplace operator where $X$ is subdivided into sub manifolds $X_{ \pm}$with common smooth boundary $Y$ of codimension $1, X=X_{-} \cup X_{+}$and $Y=X_{-} \cap X_{+}$. On int $X_{-}$we pose Dirichlet, on int $X_{+}$Neumann conditions. This problem has been investigated for a very long time, see Zaremba [61] and the subsequent development. For applications it is also interesting to admit domains with non-smooth boundary, e.g., polyhedral domains, and also interfaces with singularities.

Mixed elliptic problems can be studied from the point of view of pseudo-differential analysis with symbolic structures reflecting not only the operators in the domain and the boundary conditions but also the role of the interface $Y$ which is here interpreted as an edge embedded on the boundary $X$. With the Zaramba problem we associate a stratification

$$
s(M)=\left(s_{0}(M), s_{1}(M), s_{2}(M)\right)
$$

of $M$, where $M=s_{0}(M) \cup s_{1}(M) \cup s_{2}(M)$ for the strata $s_{2}(M):=Z, s_{1}(M):=Y \backslash Z, s_{0}(M):=M \backslash Y$. Those are smooth manifolds of different dimension. According to the general philosophy of pseudo-differential operators $A$ on such spaces, especially, differential operators, we observe a principal symbolic hierarchy

$$
\sigma(A):=\left(\sigma_{0}(A), \sigma_{1}(A), \sigma_{2}(A)\right)
$$


that determines the ellipticity. Generalities on operators on stratified spaces $M$ of some singularity order $k \in \mathbb{N}$ are developed in [46], [48]. Here $k=0$ indicates smoothness, $k=1$ conical or edge singularities, $k=2$ corners of second order, etc.. The components of (5.2) are associated with the strata in (5.1). In particular, $\sigma_{0}(A)$ is the standard homogeneous principal symbol of $A$ over $s_{0}(M)$, moreover, $\sigma_{1}(A)$ is the boundary symbol, in the present case referring to int $Y_{ \pm}$, and $\sigma_{2}(A)$ is the edge symbol. For instance, if $A$ is the Laplacian $\sum_{j=1}^{n} \frac{\partial^{2}}{\partial x_{j}^{2}}$ in $\mathbb{R}_{+}^{n}=\left\{x=\left(x_{1}, \ldots, x_{n}\right) \in \mathbb{R}^{n}: x_{n}>0\right\}$, we have $\sigma_{0}(A)=-|\xi|^{2}$ with $\xi$ being the covariable of $x$,

$$
\sigma_{1}(A)(\eta)=-|\eta|^{2}+\frac{\partial^{2}}{\partial x_{n}^{2}}
$$

where $\eta$ is the covariable of $y=\left(x_{1}, \ldots, x_{n-1}\right) \in \mathbb{R}^{n-1}$ when we represent the operator in local coordinates $x=\left(y, x_{n}\right)$ close to the boundary, with $x_{n} \in \overline{\mathbb{R}}_{+}$being the local variable normal to the boundary. We see that (5.3) is operator valued, and we take it as a family of operators $H^{s}\left(\mathbb{R}_{+}\right) \rightarrow H^{s-2}\left(\mathbb{R}_{+}\right)$parametrised by $\eta \neq 0$ (and $s \in \mathbb{R}$ not too small, see the explanations in [47] with respect to the interface). Finally, if we locally represent $Z$ as the hyperplane defined by $x_{n-1}=0, x_{n}=0$ in $x=\left(z, x_{n-1}, x_{n}\right) \in \mathbb{R}_{+}^{n}$ with $\zeta \in \mathbb{R}^{n-2}$ being the covariable of $z \in \mathbb{R}^{n-2}$, then the symbol $\sigma_{2}(A)(\zeta)$ is the family of operators

$$
\sigma_{2}(A)(\zeta)=-|\zeta|^{2}+\frac{\partial^{2}}{\partial x_{n-1}^{2}}+\frac{\partial^{2}}{\partial x_{n}^{2}}
$$

operating as $H^{s}\left(\mathbb{R}_{+}^{2}\right) \rightarrow H^{s-2}\left(\mathbb{R}_{+}^{2}\right)$, parametrised by $\zeta \neq 0$ (and $s \in \mathbb{R}$ again not too small), where

$$
\mathbb{R}_{+}^{2}=\left\{\left(x_{n-1}, x_{n}\right) \in \mathbb{R}^{2}: x_{n-1} \in \mathbb{R}, x_{n}>0\right\}
$$

The idea of the analysis of elliptic boundary value problems is to add conditions (such as Dirichlet or Neumann conditions) over $s_{1}(M)$ and to understand the nature of parametrices. As soon as we have no jump of the boundary conditions and impose the Shapiro-Lopatinskij condition then we are in the framework of "smooth" elliptic boundary value problems. The boundary operators also have a symbolic structure, and there is a well-known pseudo- differential calculus of boundary value problems, cf. Boutet de Monvel [7] and the material of Section 4 , that contains the elliptic elements themselves together with their parametrices. In this case it is adequate to consider $2 \times 2$ block matrices (4.16) with $A$ in the upper left corner, in general, together with so-called Green operators. Moreover, $T$ represents the boundary (or trace) condition while an additional potential operator $K$ appears, and $Q$ is a pseudodifferential operator on the boundary. The boundary symbolic map $\sigma_{1}$ then also applies to the other entries in (4.16).

In the Subsection 5.2, we analyze the first order pseudo-differential operator $R$ that is obtained by reducing the Neumann boundary condition to the boundary. The operator $R$, often 
referred to as the Dirichlet-to-Neumann operator, has been widely studied by many authors. Here we look at $X_{+}$and realize $R$ by a specific edge quantization as an element of the edge pseudo-differential calculus with $Y$ as the edge, operating in weighted edge spaces.

Moreover, as we have seen in Chang, Habal, and Schulze [10], the Dirichlet-to-Neumann operator $R$ has no the transmission property with respect to any interface on $X$. Therefore, we cannot expect that the truncated operator $\mathrm{r}^{+} R \mathrm{e}^{+}$induces continuous maps $H^{s}\left(\mathrm{int} X_{+}\right) \rightarrow$ $H^{s-1}$ (int $X_{+}$) (say, for $s>-1 / 2$ ). Hence, this also provides us a good example of BVPs with no transmission property

\subsection{The Dirichlet-to-Neumann operator for the Zaremba problem}

Let $M$ be the closure of a smooth bounded domain in $\mathbb{R}^{n}$ with smooth boundary $\partial M=X$. We reduce boundary problems in $M$ to the boundary, first in terms of operators in Boutet de Monvel's algebra [7] and then by using tools from the calculus of BVPs on a manifold with con-

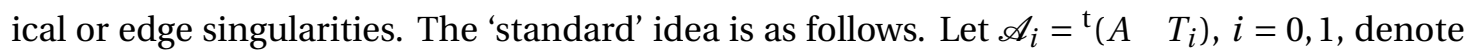
the row matrix operators representing two elliptic BVPs for an elliptic operator $A$ with trace (or boundary) operators representing boundary conditions satisfying the Shapiro-Lopatinskij condition. Assume that $A$ is a second order elliptic differential operator in $\mathbb{R}^{n}$ with smooth coefficients, in the simplest case the Laplacian and $T_{0} u:=\left.u\right|_{X}$ and $T_{1} u:=\left.\partial_{v} u\right|_{X}$ are Dirichlet and Neumann conditions, respectively, with $\partial_{v}$ being the derivative normal to the boundary. For convenience we start with the assumption that $\mathscr{A}_{0}$ is invertible, say, as an operator

$$
\mathscr{A}_{0}: C^{\infty}(M) \rightarrow \stackrel{C^{\infty}(M)}{\oplus}
$$

(as for the Laplacian and in numerous other cases), and by

$$
\mathscr{P}_{0}=\left(\begin{array}{ll}
P_{0} & K_{0}
\end{array}\right)
$$

we denote its inverse. In particular, we have $A P_{0}=1, A K_{0}=0$. Roughly speaking, $P_{0}$ is Green's operator and $K_{0}$ is the Poisson kernel of this boundary value problem. This yields

$$
\mathscr{A}_{1} \mathscr{P}_{0}=\left(\begin{array}{cc}
1 & 0 \\
T_{1} P_{0} & T_{1} K_{0}
\end{array}\right)
$$

where $T_{1} K_{0}$ is often called the Dirichlet-to-Neumann operator.

To illustrate phenomena we consider the case $A=\Delta$. However, the ideas here can be applied to more general elliptic operators $A$. We have

$$
R:=T_{1} K_{0} \in L_{\mathrm{cl}}^{1}(X), \quad \sigma_{\psi}(R)(\eta)=c|\eta|
$$


for a constant $c$; here $\sigma_{\psi}(\cdot)$ denotes the homogeneous principal symbol of the respective operator an $\eta$ the covariable on $X$ (the absolute value refers to a Riemannian metric on the boundary). The operator $R$ is elliptic, as we see from (5.6). In general the ellipticity of operators obtained by reducing an elliptic BVP to the boundary follows from the ellipticity of both factors in (5.5). Explicit computations for other concrete BVPs reduced to the boundary may be found in [24, page 26].

In more complicated situations below, i.e., when we replace $\mathscr{A}_{1}$ by a mixed boundary problem with jumps of the conditions along an interface $Y$ of codimension 1 on the boundary, the intention will be (similarly as in the smooth case) to express parametrices within a controlled operator algebra with symbolic structure. In the simplest case there is no jump at all, i.e., we have a reduction of $\mathscr{A}_{1}$ to the boundary by means of $\mathscr{A}_{0}$ in the form (5.5). The idea is then to construct a parametrix $\mathscr{A}_{1}^{(-1)}=: \mathscr{P}_{1}$ of $\mathscr{A}_{1}$ in terms of the known parametrix (or inverse) $\mathscr{P}_{0}$ of $\mathscr{A}_{0}$ and a parametrix $R^{(-1)}$ of $R$. First we obtain a parametrix of (5.5) as

$$
\left(\mathscr{A}_{1} \mathscr{P}_{0}\right)^{(-1)}=\left(\begin{array}{cc}
1 & 0 \\
T_{1} P_{0} & T_{1} K_{0}
\end{array}\right)^{(-1)}=\left(\begin{array}{cc}
1 & 0 \\
-R^{(-1)} T_{1} P_{0} & R^{(-1)}
\end{array}\right) .
$$

Then $\mathscr{P}_{1}$ itself follows in the form

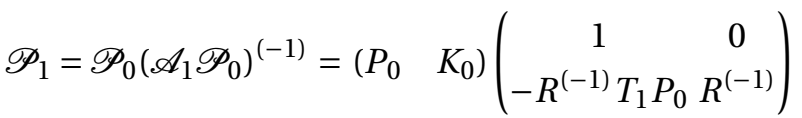

$$
\begin{aligned}
& =\left(P_{0}-K_{0} R^{(-1)} T_{1} P_{0} \quad K_{0} R^{(-1)}\right)=:\left(\begin{array}{ll}
P_{1} & K_{1}
\end{array}\right) \text {. }
\end{aligned}
$$

In the consideration below we interpret $\mathscr{A}_{0}$ and $\mathscr{A}_{1}$ as continuous operators

$$
\mathscr{A}_{0}=\left(\begin{array}{c}
A \\
T_{0}
\end{array}\right): H^{s}(\operatorname{int} M) \rightarrow \begin{gathered}
H^{s-2}(\operatorname{int} M) \\
H^{s-1 / 2}(X)
\end{gathered},
$$

and

$$
\mathscr{A}_{1}=\left(\begin{array}{c}
A \\
T_{1}
\end{array}\right): H^{s}(\operatorname{int} M) \rightarrow \begin{gathered}
H^{s-2}(\operatorname{int} M) \\
\oplus \\
H^{s-3 / 2}(X)
\end{gathered},
$$

first for $s>3 / 2$. By results in [7] or [35], we know that the operators $\mathscr{P}_{i}=\left(P_{i} K_{i}\right)$ belong to Boutet de Monvel's calculus of pseudo-differential BVPs with the transmission property at the boundary, more precisely, $P_{i}=F+G_{i}$ with $F=\mathrm{r}^{+} \tilde{F} \mathrm{e}^{+}$being the truncation of a fundamental solution (or a parametrix) $\tilde{F}$ of $\Delta$ in $\mathbb{R}^{n}$ to int $M$. Here $\mathrm{e}^{+}$is the operator of extension by zero from int $M$ to $\mathbb{R}^{n}$ and $\mathrm{r}^{+}$the operator of restriction of distributions to int $M$. Moreover, $G_{i}$ is a Green operator and $K_{i}$ a potential operator in Boutet de Monvel's calculus. The advantage of this viewpoint is that we have the principal symbolic structure of such operators, more 
precisely, the pair $\sigma=\left(\sigma_{0}, \sigma_{1}\right)$ of symbols where $\sigma_{0}$ is the standard homogeneous principal symbol of operators over $M$ (smooth up to the boundary) while $\sigma_{1}$ represents the so-called principal boundary symbol. Moreover, we can freely compose operators in Boutet de Monvel's algebra; this was done in (5.5) as well as in (5.7), and the symbols are (componentwise) multiplicative. Concerning $T_{i}$ and the other operators in lower left corners, those are trace operators in Boutet de Monvel's calculus, and they have boundary symbols as well. Parametrices $\mathscr{P}_{i}$ of $\mathscr{A}_{i}$ belong to the inverted symbolic components, i.e., $\sigma\left(\mathscr{A}_{i}\right)^{-1}=\sigma\left(\mathscr{P}_{i}\right), i=0,1$, as a componentwise relation.

In the construction of $R=T_{1} K_{0}$, the reduction of the Neumann condition to the boundary by means of the solution of the Dirichlet problem, it is not essential that $\mathscr{P}_{0}$ is the inverse of $\mathscr{A}_{0}$. We are mainly interested in Fredholm operators between the chosen Sobolev spaces, and it suffices to employ $\mathscr{P}_{0}$ as a parametrix of $\mathscr{A}_{0}$. By interchanging the role of $\mathscr{A}_{0}$ and $\mathscr{A}_{1}$ for a parametrix $\mathscr{P}_{1}$ of the Neumann problem we obtain $\mathscr{A}_{0} \mathscr{P}_{1}=\left(\begin{array}{cc}1 & 0 \\ T_{0} P_{1} & T_{0} K_{1}\end{array}\right)$ modulo a smoothing operator in Boutet de Monvel's calculus. Let us ignore such remainders in the following compositions. Then the composition $\left(\mathscr{A}_{0} \mathscr{P}_{1}\right)\left(\mathscr{A}_{1} \mathscr{P}_{0}\right)=\left(\begin{array}{cc}1 & 0 \\ T_{0} P_{1} & T_{0} K_{1}\end{array}\right)\left(\begin{array}{cc}1 & 0 \\ T_{1} P_{0} & T_{1} K_{0}\end{array}\right)$ is the identity modulo a smoothing operator, which implies that $R^{(-1)}:=T_{0} K_{1} \in L_{\mathrm{cl}}^{-1}(X)$ is a parametrix of $R=T_{1} K_{0} \in L_{\mathrm{cl}}^{1}(X)$. Let us now replace $\mathscr{A}_{1}$ by the Zaremba problem

$$
\mathscr{A}_{\mathrm{m}}:=\left(\begin{array}{c}
A \\
D_{-} \\
N_{+}
\end{array}\right): C^{\infty}(M) \rightarrow C^{\infty}\left(X_{-}\right),
$$

for $D_{-} u:=\left.\left(T_{0} u\right)\right|_{\operatorname{int} X_{-}}, N_{+} u:=\left.\left(T_{1} u\right)\right|_{\operatorname{int} X_{+}}$where $X:=\partial M$ is subdivided into submanifolds $X_{+}$, $X_{-}$with common boundary $Y=X_{+} \cap X_{-}$, in the simplest case assumed to be smooth. Then by virtue of (5.5) together with $\mathscr{A}_{0} \mathscr{P}_{0}=\mathrm{id}_{H^{s-2}(G)}$ we obtain

$$
\begin{array}{ll}
D_{-} K_{0}=1 & \text { on } \operatorname{int} X_{-}, \\
N_{+} K_{0}=R & \text { on } \operatorname{int} X_{+} .
\end{array}
$$

Thus the reduction of the mixed condition gives rise to $R$ on the manifold $X_{+}$with boundary $\partial X_{+}=: Y$ and the identity on $X_{-}$. The relation (5.9) shows that in the reduction of the mixed conditions $T:={ }^{\mathrm{t}}\left(D_{-} N_{+}\right)$to the boundary the resulting operator on the boundary has a jump; it is equal to the Dirichlet-to-Neumann operator $R$ on $X_{+}$and to the identity on $X_{-}$. Apart from the identity one of the main tasks is to solve a boundary value problem for $R$ on $X_{+}$ with $Y=\partial X_{+}$as the boundary. However, $R$ fails to have the transmission property at the 
boundary, it has in fact, the anti-transmission property, cf. [50] and it is hard to imagine that Boutet de Monvel's calculus extends to this case. This problem has been studied by many mathematicians, cf. Vishik, Eskin [60] or Eskin [18], see also [24], [36], and [47]. In the present paper we develop an approach that is based on a Mellin quantisation, already indicated in Dines, Liu, and Schulze [15]. Here we employ this method for the construction of a parametrix of (5.8) within a variant of edge calculus where $Y$ plays the role of an edge. For the complete solution of this problem, we refer to the paper [10].

\subsection{The Dirichlet-to-Neumann operator for the $\bar{\partial}$-Neumann problem}

Let $\Omega$ be a bounded domain in $\mathbb{C}^{n+1}$ with $\mathscr{C}^{\infty}$ boundary, i.e., there exists a real-valued function $\rho \in C^{\infty}(\bar{\Omega})$ such that

$$
\partial \Omega=\left\{z \in \mathbb{C}^{n+1}: \rho(z)=0\right\}
$$

with $d \rho(z) \neq 0, \forall z \in \partial \Omega$.

One of the basic problem in several complex variables is to solve the inhomogeneous Cauchy-Riemann equation

$$
\bar{\partial} U=f \quad \text { in } \Omega
$$

with "good" bounds on $\Omega$, where $f$ is a given $(0,1)$-form $f=\sum_{j=1}^{n+1} f_{j} d \bar{\omega}_{j}$. Obvious, the righthand side of (5.10) has $n+1$ data but the left-hand side of (5.10) has only one function. Therefore, the system (5.10) is over-determined when $n \geq 1$. It follows that the equation (5.10) is solvable only when $f$ satisfies a consistence condition, i.e., $\bar{\partial} f=0$. Moreover, solution for the equation (5.10) is highly non-unique. Suppose $U$ is a solution of (5.10), then $U+F$ is also a solution whenever $F \in \mathscr{H}(\Omega)$ where $\mathscr{H}(\Omega)$ is the set of all holomorphic functions defined on $\Omega$.

Denote $\mathscr{A}^{2}(\Omega)=L^{2}(\Omega) \cap \mathscr{H}(\Omega)$ the Bergman space. Then we can fine a "canonical solution" $U$ by requiring that

$$
U \perp \mathscr{H}(\Omega) \text { in } \mathscr{A}^{2}(\Omega)
$$

which minuses the $L^{2}$-norm among all solutions. In order to find the canonical solution, let us consider a first-order differential operator $D$ and $\phi, \psi \in \mathscr{C}^{\infty}(\bar{\Omega})$, then the formal adjoint $D^{*}$ of $D$ can be defined as follows

$$
\int_{\Omega}(D \phi) \bar{\psi} d V=\int_{\Omega} \phi \overline{\left(D^{*} \psi\right)} d V+\int_{\partial \Omega} \phi \overline{\left(A^{\sharp} \psi\right)} d \sigma,
$$

where $A^{\sharp}$ is a 0 th-order operator defined on $\partial \Omega$. In our case $D=\bar{\partial}$ is the Cauchy-Riemann operator. Hence,

$$
\operatorname{dom}\left(\bar{\partial}^{*}\right)=\left\{\psi \in \mathscr{C}^{\infty}(\bar{\Omega}): A^{\sharp} \psi=0 \text { on } \partial \Omega\right\} .
$$


Note that with $U=\bar{\partial}^{*} u$, then for $F \in \mathscr{H}(\Omega)$

$$
\left\langle\bar{\partial}^{*} u, F\right\rangle=\langle u, \bar{\partial} F\rangle=0 .
$$

This means that is we solve the equation

$$
\bar{\partial} \bar{\partial}^{*} u=f, \quad u \in \operatorname{dom}\left(\bar{\partial}^{*}\right),
$$

then we solve (5.10) with a canonical solution.

In fact, problem (5.11) is equivalent to the case $\bar{\partial} f=0$ of the system

$$
\begin{aligned}
& \square u=\left(\bar{\partial} \bar{\partial}^{*}+\bar{\partial}^{*} \bar{\partial}\right) u=f, \\
& u \in \operatorname{dom}\left(\bar{\partial}^{*}\right), \bar{\partial} u \in \operatorname{dom}\left(\bar{\partial}^{*}\right) .
\end{aligned}
$$

To see that, $0=\bar{\partial} f=\bar{\partial}\left(\bar{\partial} \bar{\partial}^{*}+\bar{\partial}^{*} \bar{\partial}\right) u=\bar{\partial} \bar{\partial}^{*} \bar{\partial} u$, and so

$$
0=\left\langle\bar{\partial} u, \bar{\partial} \bar{\partial}^{*} \bar{\partial} u\right\rangle=\left\langle\bar{\partial}^{*} \bar{\partial} u, \bar{\partial}^{*} \bar{\partial} u\right\rangle \Rightarrow \bar{\partial}^{*} \bar{\partial} u=0 .
$$

The system (5.12) is not over-determined. For general $u \in B^{(0, q)}(\Omega)$, the system (5.12) is called that " $\bar{\partial}$-Neumann problem". Here $B^{(0, q)}(\Omega)$ is the collection of all $(0, q)$ forms defined on $\Omega$. The formalism of the $\bar{\partial}$-Neumann problem was introducing by D.C. Spencer in the early 1950's. Under certain assumptions on $\Omega$, J.J. Kohn [28] obtained the first result in 1963 and 1964:

$$
\|u\|_{H^{s+1}(\Omega)} \leq C\left(\|f\|_{H^{s}(\Omega)}+\|u\|_{L^{2}(\Omega)}\right), \quad s \in \mathbb{R} .
$$

Moreover, the estimate is sharp in $L^{2}$. Unlike the elliptic case, the solution $u$ does not gain two in all directions. Therefore, the system (5.12) has great interests from the point of view of partial differential equations.

There are essentially three aspects to this problem:

•. Existence of solutions;

•. Find the solving operator $N$ (and hence $\bar{\partial}^{*} N$ );

-. Sharp estimates for $N$ and $\bar{\partial}^{*} N$.

Let $g$ be a smooth Hermitian metric on $\mathbb{C}^{n+1}$. Then there is an open neighborhood $V$ of $\partial \Omega$ such that if $\rho$ denotes a signed geodesic distance in the metric $g$ to $\partial \Omega$, then

$$
\begin{aligned}
& \Omega^{+}=\Omega \cap V=\{z \in U: \rho(z)>0\} \\
& \nabla \rho(z) \neq 0 \text { for all } z \in \partial \Omega \cap V .
\end{aligned}
$$


We choose a smooth orthogonal basis for $(0,1)$-form on $V$, given by $\bar{\omega}_{1}, \ldots, \bar{\omega}_{n+1}$ with

$$
\bar{\omega}_{n+1}=\sqrt{2} \bar{\partial} \rho .
$$

We let $\bar{Z}_{1}, \ldots, \bar{Z}_{n+1}$ be the dual basis of antiholomorphic vector fields on $V$. Then

$$
Z_{1}, \ldots, Z_{n}, \bar{Z}_{1}, \ldots, \bar{Z}_{n}
$$

are tangential to $\partial \Omega$. If $\frac{\partial}{\partial \rho}$ is the vector field dual to the one form $d \rho$, then

$$
Z_{n+1}=\frac{1}{\sqrt{2}} \frac{\partial}{\partial \rho}+i T
$$

is the complex normal.

Let $\left\{Z_{j}, \bar{Z}_{j}, T\right\}_{j=1, \ldots, n}$ be a basis of the complex tangent bundle of $\partial \Omega \cap V$. The vector fields $\left[Z_{j}, \bar{Z}_{k}\right]$ in terms of this basis is give by

$$
\left[Z_{j}, \bar{Z}_{k}\right]=c_{j k} \sqrt{-1} T+\sum_{\ell=1}^{n} a_{j k}^{\ell} Z_{\ell}+\sum_{\ell=1}^{n} b_{j k}^{\ell} \bar{Z}_{\ell} .
$$

The Hermitian form $\left(c_{j k}\right)$ is called the Levi form.

The domain $\Omega$ is called pseudoconvex if each point of $\Omega$ has a neighborhood on which the vector field $T$ can be chosen so that $\left(c_{j k}\right) \geq 0$. If the Levi form $\left(c_{j k}\right)>0$, then $\Omega$ is called strongly pseudoconvex.

To simplify our discussion, we just assume that $\Omega$ is strongly pseudoconvex in this subsection. In this case, $\Omega$ has a foliation. By a result of Chern and Moser [14], $\Omega$ and $\partial \Omega$ can be locally approximated by the "Siegel upper half-space" and the "Heisenberg group" respectively in $\mathbb{C}^{n+1}$. Readers can consult Stein's book [58] for background of Heisenberg group and its connection with analysis in several complex variables. The domain consists of all $z \in \mathbb{C}^{n+1}$, $n \geq 1$, so that

$$
\Omega=\left\{\left(z_{1}, \ldots, z_{n}, z_{n+1}\right) \in \mathbb{C}^{n+1}: \operatorname{im}\left(z_{n+1}\right)>\sum_{j=1}^{n}\left|z_{j}\right|^{2}\right\} .
$$

Its boundary is the "paraboloid"

$$
\partial \Omega=\left\{\left(z_{1}, \ldots, z_{n}, z_{n+1}\right) \in \mathbb{C}^{n+1}: \operatorname{im}\left(z_{n+1}\right)=\sum_{j=1}^{n}\left|z_{j}\right|^{2}\right\} .
$$

In this case, the vector fields $Z_{j}$ can be written as

$$
Z_{j}=\frac{\partial}{\partial z_{j}}+i \bar{z}_{j} \frac{\partial}{\partial t}, \quad j=1, \ldots, n
$$


and the complex normal $Z_{n+1}$ is

$$
Z_{n+1}=\frac{1}{\sqrt{2}}\left(\frac{\partial}{\partial \rho}+i T\right)=\frac{1}{\sqrt{2}}\left(\frac{\partial}{\partial \rho}+i \frac{\partial}{\partial t}\right)
$$

where $\rho=\operatorname{im}\left(z_{n+1}\right)-\sum_{j=1}^{n}\left|z_{j}\right|^{2}$ is the "height" function and $T=\frac{\partial}{\partial t}$ is the "missing direction".

Because the vector fields split into tangential and normal part, we may consider a $(0,1)$ form $u$ as follows:

$$
u=\sum_{j=1}^{n+1} u_{j} \bar{\omega}_{j}=\sum_{j=1}^{n} u_{j} \bar{\omega}_{j}+u_{n+1} \bar{\omega}_{n+1} .
$$

Then the $\bar{\partial}$-Neumann problem is the following boundary value problem:

$$
\begin{aligned}
& \square u=f \quad \text { in } \quad \Omega \\
& u_{n+1}=0 \quad \text { on } \quad \partial \Omega \\
& \bar{Z}_{n+1}\left(u_{j}\right)-[S(u)]_{j, n+1}=0 \quad \text { on } \quad \partial \Omega
\end{aligned}
$$

for $j=1, \ldots, n$. Here

$$
[S(u)]_{j, n+1}=\sum_{\ell=1}^{n} \bar{s}_{j, n+1}^{\ell} u_{j}, \quad j=1, \ldots, n
$$

and the matrix $S$ is defined by the equations

$$
\bar{\partial} \bar{\omega}_{\ell}=\sum_{j<k} \bar{s}_{j, k}^{\ell} \bar{\omega}_{j} \wedge \bar{\omega}_{k}
$$

Then the operator $\square$ which is basically the complex Laplacian which can be written as

$$
\begin{aligned}
\square u= & {\left[\begin{array}{ccccc}
\square_{1} & 0 & \cdots & 0 & 0 \\
0 & \square_{2} & \cdots & 0 & 0 \\
\cdots & & & \\
0 & 0 & \cdots & \square_{n} & 0 \\
0 & 0 & \cdots & 0 & \square_{n+1}
\end{array}\right] u } \\
& +\left(h_{n+1} \mathbf{I}_{n+1}+S^{t}\right)\left(\bar{Z}_{n+1} u\right)-\left(\bar{S}\left(Z_{n+1} u\right)\right)+\varepsilon(Z, \bar{Z}) u+\varepsilon(u)
\end{aligned}
$$

where $h_{n+1}$ is a smooth function which comes from the volume element. $\varepsilon(Z, \bar{Z}) u$ represents terms of first derivatives of $u$ along horizontal directions and $\varepsilon(u)$ represents terms of $u$ multiplying by smooth functions. Here

$$
\square_{\ell}=-\frac{1}{2} \sum_{j=1}^{n}\left(Z_{j} \bar{Z}_{j}+\bar{Z}_{j} Z_{j}\right)-Z_{n+1} \bar{Z}_{n+1}+\left(\sum_{j=1}^{n} \lambda_{j}-2 \lambda_{\ell}\right) i T
$$

for $\ell=1,2, \ldots, n$ and

$$
\square_{n+1}=-\frac{1}{2} \sum_{j=1}^{n}\left(Z_{j} \bar{Z}_{j}+\bar{Z}_{j} Z_{j}\right)-Z_{n+1} \bar{Z}_{n+1}+\left(\sum_{j=1}^{n} \lambda_{j}\right) i T
$$


We shall construct a solving operator $N=\left(N_{1}, \ldots, N_{n+1}\right)$ for the $\bar{\partial}$-Neumann problem so that modulo smooth error $u_{j}=N_{j}\left(f_{j}\right)$ for $j=1,2, \ldots, n+1$.

The "normal" component $u_{n+1}$ is the solution of a Dirichlet problem for an elliptic operator:

$$
\begin{array}{ccc}
\square_{n+1} u_{n+1}=f_{n+1} & \text { in } \quad \Omega \\
u_{n+1}=0 & \text { on } & \partial \Omega
\end{array}
$$

From previous discussion, the structure of this solution is well understood.

We note that a parametric for the $(n+1)$-component $N_{n+1}$ of the Neumann operator $N$ is given by

$$
N_{n+1}(z, t ; \rho)=G\left((w, s)^{-1} \cdot(z, t) ; \rho-\mu\right)-G\left((w, s)^{-1} \cdot(z, t) ; \rho+\mu\right)
$$

where $G(z, t ; \rho)=\frac{2^{n-1} \Gamma(n)}{\pi^{n+1}}\left(2|z|^{2}+t^{2}+\rho^{2}\right)^{-n}$. Hence,

$$
\left\|\frac{\partial^{2} N_{n+1}(f)}{\partial x_{j} \partial x_{\ell}}\right\|_{H^{s}(\Omega)} \leq C_{p}\|f\|_{H^{s}(\Omega)}, \quad s \in \mathbb{R},
$$

for $1 \leq j, \ell \leq 2 n+2$, i.e., $N_{n+1}$ gains 2 in all directions.

Now we are left with solving the following nonelliptic boundary problem: Given $f$ on $\Omega$, find a function $u$ on $\bar{\Omega}$ such that

$$
\begin{aligned}
& \frac{\partial^{2} u}{\partial \rho^{2}}+\frac{\partial^{2} u}{\partial t^{2}}+\Delta_{\ell} u=f \text { in } \Omega \\
& \left(\frac{\partial u}{\partial \rho}-i \frac{\partial u}{\partial t}\right)(x, t, 0)=0
\end{aligned}
$$

where

$$
\Delta_{\ell}=\frac{1}{2} \sum_{j=1}^{n}\left(Z_{j} \bar{Z}_{j}+\bar{Z}_{j} Z_{j}\right)+\left(\sum_{j=1}^{n} \lambda_{j}-2 \lambda_{\ell}\right) i T
$$

for $\ell=1, \ldots, n$.

In order to solve the problem (5.13), we may assume the solution $u$ is given by

$$
u(x, t ; \rho)=G(f)+P\left(u_{b}\right)
$$

where $G$ is the Green's function for the Dirichlet problem and $P$ is the Poisson operator. Here $u_{b}$ is the "boundary value" of $u$ which we need to determine.

On the other hand, $u$ satisfies the $\bar{\partial}$-Neumann boundary conditions, i.e., $R \bar{Z}_{n+1}(u)=0$, $j=1, \ldots, n$ where $R$ is the restriction operator to $\partial \Omega$. Therefore,

$$
0=R \bar{Z}_{n+1}(u)=R \bar{Z}_{n+1} G(f)+R \bar{Z}_{n+1} P\left(u_{b}\right)
$$


i.e.,

$$
\square_{+}\left(u_{b}\right)=R \bar{Z}_{n+1} P\left(u_{b}\right)=-R \bar{Z}_{n+1} G(f) .
$$

The operator $\square_{+}$is called the Dirichlet-to-Neumann operator associated to the $\bar{\partial}$-Neumann problem. This is a 1st order pseudo-differential operator defined on $\partial \Omega$. Hence, in order to solve the $\bar{\partial}$-Neumann problems reduces to invert the operator $\square_{+}$.

After detailed calculation, one can see that he principal symbol of the operator $\square_{+}$is

$$
\sigma\left(\square_{+}\right)=\frac{1}{\sqrt{2}}(\tau-\Delta)-\frac{1}{\sqrt{2}}\left(2 \lambda_{j}-\sum_{k=1}^{n} \lambda_{k}\right) \frac{\tau}{\Delta}
$$

where $\tau=\sigma(-i T)$ and

$$
\Delta=\sqrt{\tau^{2}+2 \sum_{j=1}^{n}\left|\sigma\left(Z_{j}\right)\right|^{2}} .
$$

Obviously, $\square_{+}$is elliptic when $\tau<0$ but doubly characteristic on half of the line bundle

$$
\Sigma^{+}=\{(z, t ; \xi, \tau): \tau>\Delta\}
$$

on the cotangent bundle $T^{*}(\partial \Omega)$.

On the other hand, we may also construct the Dirichlet-to-Neumann operator $\square_{\text {- of the }}$ $\bar{\partial}$-Neumann problem on

$$
\bar{\Omega}^{-}=\{z \in U: \rho(z) \leq 0\} .
$$

The principal symbol of $\square_{-}$is

$$
\sigma\left(\square_{-}\right)=\frac{1}{\sqrt{2}}(\tau+\Delta)+\frac{1}{\sqrt{2}}\left(2 \lambda_{j}-\sum_{k=1}^{n} \lambda_{k}\right) \frac{\tau}{\Delta} .
$$

It is a 1st order pseudo-differential operator doubly characteristic on the half of the line bundle

$$
\Sigma^{-}=\{(z, t ; \xi, \tau): \tau<-\Delta\}
$$

but elliptic on the characteristics of $\square_{+}$. An important phenomena is

$$
\square_{+} \circ \square_{-}=-\square_{b}+\text { zero order terms }
$$

and

$$
\square_{-} \circ \square_{+}=-\square_{b}+\text { zero order terms. }
$$

Here $\square_{b}$ is the sub-Laplacian on $(0,1)$-forms defined on the boundary $\partial \Omega$. More precisely,

$$
\square_{b}=\left[\begin{array}{cccc}
\square_{1}^{\prime} & 0 & \cdots & 0 \\
0 & \square_{2}^{\prime} & \cdots & 0 \\
\cdots & & & \\
0 & 0 & \cdots & \square_{n}^{\prime}
\end{array}\right]+\mathscr{A}
$$

with

$$
\square_{\ell}^{\prime}=-\frac{1}{2} \sum_{j=1}^{n}\left(Z_{j} \bar{Z}_{j}+\bar{Z}_{j} Z_{j}\right)+i\left(\sum_{k=1}^{n} \lambda_{k}-2 \lambda_{\ell}\right) T
$$


for $\ell=1, \ldots, n$ and $\mathscr{A}=\left[\mathscr{A}_{\alpha \beta}\right]$ of the form

$$
\mathscr{A}_{\alpha \beta}=\sum_{k=1}^{n} a_{\alpha \beta}^{k} Z_{k}+\sum_{k=1}^{n} b_{\alpha \beta}^{k} \bar{Z}_{k}+c_{\alpha \beta} .
$$

In order to find a full parametrix for the $\bar{\partial}$-Neumann problem, one needs to invert $\square_{b}$ on $\partial \Omega$.

When $n>1$, the sub-Laplacian $\square_{b}$ has an inverse $\mathbf{K}$ by a result of Folland-Stein [20] (see also Beals and Greiner [5] and Berenstein-Chang-Tie [6]) and then the $\bar{\partial}$-Neumann problem has a parametrix

$$
N_{j}=G+P\left(-\mathbf{K} \square_{-} R \bar{Z}_{n+1} G\right)+S_{-\infty}, \quad j=1, \ldots, n
$$

where $S_{-\infty}$ is a smoothing operator.

When $n=1$, we know that

$$
\begin{aligned}
\square(\varphi) & =-\frac{1}{2}\left(Z_{1} \bar{Z}_{1}+\bar{Z}_{1} Z_{1}\right)-i T(\varphi)+\varepsilon\left(Z_{1}, \bar{Z}_{1}, \varphi\right) \\
& =-Z_{1} \bar{Z}_{1}(\varphi)+\varepsilon\left(Z_{1}, \bar{Z}_{1}, \varphi\right)
\end{aligned}
$$

In this case, $\square_{b}$ is not invertible since it is intimately connected with the non-solvability of the Lewy equation. However, by a result of Greiner and Stein [22], one can construct an operator $\widetilde{\mathbf{K}}$, so that

$$
\widetilde{\mathbf{K}} \square_{b}=\square_{b} \widetilde{\mathbf{K}}=I-\overline{\mathscr{C}}_{b}
$$

where $\mathscr{C}_{b}$ is the Cauchy-Szegö projection.

Let us consider 0th-order pseudo-differential operators: $E^{+}$and $E^{-}$with symbols in the class $S_{1,0}^{0}$ such that the principal symbol of $E^{+}$equals to 1 on the set

$$
\left\{\Delta<\frac{1}{4} \tau\right\}
$$

and whose principal symbol equals to 0 on the set

$$
\left\{\Delta>\frac{1}{2} \tau\right\}
$$

The important fact is that

$$
E^{+} \circ \overline{\mathscr{C}}_{b}=\overline{\mathscr{C}}_{b} \circ E^{+}=0, \quad \text { approximately, }
$$

and hence the projection $\overline{\mathscr{C}}_{b}$ is subordinate to projection $E^{-}=I-E^{+}$; moreover $\square^{+}$is elliptic away from its characteristic variety. Thus there exists a pseudo-differential operator of order -1 , $\mathscr{Q}_{E^{-}}$, so that

$$
\mathscr{Q}_{E^{-}} \circ \square^{+}=E^{-} \quad \text { approximately. }
$$

It is then easy to see that $-E^{+} \circ \bar{K} \square^{-}+\mathscr{Q}_{E^{-}}$is an approximate inverse to $\square^{+}$, i.e.,

$$
\begin{aligned}
\left(-E^{+} \circ \bar{K} \square^{-}+\mathscr{Q}_{E^{-}}\right) \square^{+} & =-E^{+} \bar{K} \square^{-} \square^{+}+\mathscr{Q}_{E^{-}} \square^{+}=-E^{+} \bar{K}\left(-\square_{b}\right)+E^{-}+T_{-1} \\
& =-E^{+}\left(-I+\overline{\mathscr{C}}_{b}\right)+E^{-}+T_{-1} \\
& =E^{+}+E^{-}+T_{-1}-E^{+} \overline{\mathscr{C}}_{b} \\
& =I-E^{+} \overline{\mathscr{C}}_{b}+T_{-1}=I+T_{-1} .
\end{aligned}
$$

Hence, the $\bar{\partial}$-Neumann problem has a parametrix (see Chang-Nagel-Stein[11]):

$$
N_{1}=G+P\left(-K^{+} \square_{-} R \bar{Z}_{2} G\right)+S_{-\infty}, \quad j=1, \ldots, n
$$


where $S_{-\infty}$ is a smoothing operator and

$$
K^{+}=-Q_{E^{-}}+E^{+} \bar{K} \circ \square^{-}
$$

where $Q_{E^{-}}$denotes the parametric for $\square^{+}$in the support of $E^{-}=I-E^{+}$, i.e.,

$$
Q_{E^{-} \circ \square^{+}}=E^{-}+T_{-\infty}
$$

Finally, under the hypothesis that $\Omega$ is a smoothly bounded, strongly pseudoconvex domain, it can be proved that the Neumann operator satisfies

•. $N: H^{s}(\Omega) \rightarrow H^{s+1}(\Omega)$;

-. $\mathscr{P}(Z, \bar{Z}) N: H^{s}(\Omega) \rightarrow H^{s}(\Omega)$. Here $\mathscr{P}(Z, \bar{Z})$ is any quadratic monomial in tangential vector fields.

As a consequence of the above subelliptic estimates, one can show that the solving operator $\bar{\partial}^{*} N$ of the canonical solution of the inhomogeneous Cauchy-Riemann equation satisfies the following estimates:

•. $\bar{\partial}^{*} N: H^{s}(\Omega) \rightarrow H^{s+\frac{1}{2}}(\Omega)$;

- $X \bar{\partial}^{*} N: H^{s}(\Omega) \rightarrow H^{s}(\Omega)$ for any tangential tangential vector fields $X$.

The above results had been generalized to many other situations, see for example, [8]. We will not discuss this here.

\section{Acknowledgement}

This research project is partially supported by an NSF grant DMS-1203845, Multi-Year Research Grant MYRG115(Y1-L4)-FST13-QT at University of Macau and Hong Kong RGC competitive earmarked research grant \#601410. The paper is based on the first part of a series of lectures which were given by the authors at the National Center for Theoretical Sciences, Hsinchu, Taiwan during May-July, 2014. They would like to express their profound gratitude to the Director of NCTS, Professor Winnie Li for her invitation and for the warm hospitality extended to them during their stay in Taiwan.

\section{References}

[1] S. Agmon, A. Douglis, and L. Nirenberg, Estimates near the boundary for solutions of elliptic partial differential equations satisfying general boundary conditions I, Comm. Pure Appl. Math., 12 (1959), 623-727.

[2] S. Agmon, A. Douglis, and L. Nirenberg, Estimates near the boundary for solutions of elliptic partial differential equations satisfying general boundary conditions II, Comm. Pure Appl. Math., 17 (1964), 35-92.

[3] M. F. Atiyah and I. M. Singer, The index of elliptic operators I, II, III, Ann. of Math. (2), 87 (1968), 483-530, 531-545, 546-604.

[4] M. F. Atiyah and R. Bott, The index problem for manifolds with boundary, Coll. Differential Analysis, Tata Institute Bombay, Oxford University Press, Oxford 1964, pp. 175-186.

[5] R. Beals and P.C. Greiner, Calculus on Heisenberg manifolds, Ann. Math. Studies, 119, Princeton University Press, Princeton, New Jersey, 1988.

[6] C. Berenstein, D.-C. Chang and J. Tie, Laguerre calculus on the Heisenberg group, AMS/IP series in Advanced Mathematics, 22, American Math. Soc. and International Press, Cambridge, Massachusetts, 2001.

[7] L. Boutet de Monvel, Boundary problems for pseudo-differential operators, Acta Math., 126 (1971), 11-51. 
[8] D.-C. Chang and C. Fefferman, On $L^{p}$ estimates of the Cauchy-Riemann equation, Harmonic Analysis in China, Kluwer Academic Publishers, Boston-London 1995, pp. 1-21.

[9] D.-C. Chang, N. Habal, and B.-W. Schulze, Quantisation on a manifold with singular edge, NCTS Preprints in Mathematics 2013-1-002, Taiwan, 2013. J. Pseudo-Differ Oper Appl., 4, 3 (2013), 317-343.

[10] D.-C. Chang, N. Habal and B.-W. Schulze, The edge algebra structure of the Zaremba problem, NCTS Preprints in Mathematics 2013-6-002, Taiwan, 2013. J. Pseudo-Differ. Oper. Appl., 5(2014), 69-155.

[11] D.-C. Chang, A. Nagel, and E.M. Stein, Estimates for the $\bar{\partial}-$ Neumann problem in pseudoconvex domains of finite type in $\mathbb{C}^{2}$, Acta Math., 169 (1992), 153-227.

[12] D.-C. Chang, T. Qian and B.-W. Schulze, Corner Boundary Value Problems, Complex Analysis and Operator Theory, 9 (2014), 1157-1210.

[13] D.-C. Chang, W. Rungrottheera and B.-W. Schulze, Recent developments on pseudo-differential operators (I), Tamkang Journal of Mathematics, 46 (2015), 1-30.

[14] S. S. Chern and J. K. Moser, Real hypersurfaces in complex manifolds, Acta Math., 133 (1974), $219-271$.

[15] N. Dines, X. Liu, and B.-W. Schulze, Edge quantisation of elliptic operators, Monatshefte für Math., 156 (2009), 233-274.

[16] C. Dorschfeldt, Algebras of pseudo-differential operators near edge and corner singularities, Math. Res. 102 , Akademie Verlag, Berlin, 1998.

[17] J. V. Egorov and B.-W. Schulze, Pseudo-differential operators, singularities, applications, Oper. Theory: Adv. Appl. 93, Birkhäuser Verlag, Basel, 1997.

[18] G. I. Eskin, Boundary value problems for elliptic pseudodifferential equations, Transl. of Nauka, Moskva, 1973, Math. Monographs, Amer. Math. Soc., 52, Providence, Rhode Island 1980.

[19] H.-J. Flad, G. Harutyunyan, R. Schneider, and B.-W. Schulze Explicit Green operators for quantum mechanical Hamiltonians.I, manuscripta math. 135 (2011), 497-519.

[20] G. B. Folland and E. M. Stein, Estimates for the $\bar{\partial}_{b}$-complex and analysis on the Heisenberg group, Comm. Pure Appl. Math., 27(1974), 429-522.

[21] I. M. Gelfand, On elliptic equations, Uspechi Math. Nauk., 15(1960), 121-132. (Russian)

[22] P. C. Greiner and E. M. Stein, Estimates for the $\bar{\partial}-$ Neumann problem, Math. Notes, 19, Princeton University Press, Princeton, New Jersey, 1977.

[23] G. Grubb, Functional calculus of pseudo-differential boundary problems, Second Edition, Birkhäuser Verlag, Boston, 1996.

[24] G. Harutjunjan and B.-W. Schulze, Elliptic mixed, transmission and singular crack problems, European Mathematical Soc., Zürich, 2008.

[25] T. Hirschmann, Functional analysis in cone and edge Sobolev spaces, Ann. Global Anal. Geom., 8(1990), 167192.

[26] L. Hörmander, Pseudo-differential operators, Comm. Pure Appl. Math., 18(1965), 501-517.

[27] D. Kapanadze and B.-W. Schulze, Crack Theory and Edge Singularities, Kluwer Academic Publ., Dordrecht, 2003.

[28] J. Kohn, Harmonic integrals on strongly pseudo-convex manifolds I and II, Ann. of Math., 78 (1963), 112-148, 79 (1964), 450-476.

[29] J. Kohn, and L. Nirenberg, An algebra of pseudo-differential operators, Comm. Pure Appl. Math., 18 (1965), 269-305.

[30] V. A. Kondratyev, Boundary value problems for elliptic equations in domains with conical points, Trudy Mosk. Mat. Obshch., 16(1967), 209-292.

[31] T. Krainer, On the inverse of parabolic systems of partial differential equations of general form in an infinite space-time cylinder, Oper. Theory Adv. Appl., 138, Adv. in Partial Differential Equations "Parabolicity, Volterra Calculus, and Conical Singularities” (Albeverio, S. and Demuth, M. and Schrohe, E. and Schulze, B.-W., eds.), Birkhäuser Verlag, Basel, 2002, pp. 93-278.

[32] J.-L. Lions, and E. Magenes, Problèmes aux limites non homogènes et applications, vol. 1, Dunod, Paris, 1968.

[33] Ya. A. Lopatinskij, On a method of reducing boundary problems for a system of differential equations of elliptic type to regular equations, Ukraïn. Math. Zh., 5 (1953), 123-151. 
[34] X. Lyu and B.-W. Schulze, Mellin operators in the edge calculus, (in preparation).

[35] S. Rempel and B.-W. Schulze, Index theory of elliptic boundary problems, Akademie-Verlag, Berlin, 1982.

[36] S. Rempel and B.-W. Schulze, Parametrices and boundary symbolic calculus for elliptic boundary problems without transmission property, Math. Nachr., 105, (1982), 45-149.

[37] S. Rempel and B.-W. Schulze, Complete Mellin and Green symbolic calculus in spaces with conormal asymptotics, Ann. Glob. Anal. Geom. 4(1986), 137-224.

[38] S. Rempel and B.-W. Schulze, Asymptotics for elliptic mixed boundary problems (pseudo-differential and Mellin operators in spaces with conormal singularity), Math. Res., 50, Akademie-Verlag, Berlin, 1989.

[39] W. Rungrottheera and B.-W. Schulze, Weighted spaces on corner manifolds, Complex Variables and Elliptic Equations, 59 (2014), 1706-1738.

[40] M. Schechter, General boundary value problems for elliptic partial differential equations, Comm. Pure Appl. Math., 12(1959), 457-486.

[41] B.-W. Schulze, Corner Mellin operators and reduction of orders with parameters, Ann. Sc. Norm. Sup. Pisa Cl. Sci. 16(1989), 1-81.

[42] B.-W. Schulze, Pseudo-differential operators on manifolds with edges, Teubner-Texte zur Mathematik 112 , Symp. “Partial Differential Equations, Holzhau 1988”, BSB Teubner, Leipzig, 1989, pp. 259-287.

[43] B.-W. Schulze, Pseudo-differential Operators on Manifolds with Singularities, North-Holland, Amsterdam, 1991.

[44] B.-W. Schulze, Pseudo-differential Boundary Value Problems, Conical Singularities, and Asymptotics, Akademie Verlag, Berlin, 1994.

[45] B.-W. Schulze, Boundary Value Problems and Singular Pseudo-differential Operators, J. Wiley, Chichester, 1998.

[46] B.-W. Schulze, Operators with symbol hierarchies and iterated asymptotics, Publications of RIMS, Kyoto University 38(2002), 735-802.

[47] B.-W. Schulze and J. Seiler, The edge algebra structure of boundary value problems, Ann. Glob. Anal. Geom., 22 (2002), 197-265.

[48] B.-W. Schulze, The iterative structure of the corner calculus, Oper. Theory: Adv. Appl., 213, Pseudo-Differential Operators: Analysis, Application and Computations (L. Rodino et al. eds.), Birkhäuser Verlag, Basel, 2011, pp. 79-103.

[49] B.-W. Schulze and Y. Wei, The Mellin-edge quantisation for corner operators, arXiv:1201.6525v1 [math.AP]. Complex Analysis and Operator Theory, published online: 27 February 2013, 10.1007/s11785-013-0289-3.

[50] B.-W. Schulze, Boundary value problems with the transmission property, Oper. Theory: Adv. Appl. 205, "Pseudo-Differential Operators: Complex Analysis and Partial Differential Equations", Birkhäuser Verlag, Basel, 2009, pp. 1-50.

[51] B.-W. Schulze and M.W. Wong, Mellin and Green operators of the corner calculus, J. Pseudo-Differ. Oper. Appl., 2(2011), 467-507.

[52] R. Seeley, Topics in pseudo-differential operators, C.I.M.E. Conference on Pseudo-differential Operators, Stresa 1968, Cremonese, Roma, 1969, pp. 167-305.

[53] J. Seiler, Continuity of edge and corner pseudo-differential operators, Math. Nachr., 205 (1999), 163-182.

[54] J. Seiler, Pseudodifferential calculus on manifolds with non-compact edges, Ph.D. thesis, University of Potsdam, 1997.

[55] M. A. Shubin, Pseudo-differential Operators and Spectral Theory, Springer-Verlag, Berlin, (1987).

[56] V. A. Solonnikov, On general boundary value problems for systems which are elliptic in the sense of A. Douglis and L. Nirenberg I, Izv. AN SSSR, 28(1964), 665-706.

[57] V. A. Solonnikov, On general boundary value problems for systems which are elliptic in the sense of A. Douglis and L. Nirenberg II, Tr. MIAN SSSR, 92(1966), 233-297.

[58] E. M. Stein, Harmonic Analysis-Real variable methods, orthogonality, and oscillatory integrals, Princeton University Press, Princeton, New Jersey, 1993.

[59] M. I. Vishik and G. I. Eskin, Convolution equations in a bounded region, Uspekhi Mat. Nauk, 20(1965), 89-152. 
[60] M. I. Vishik and G. I. Eskin, Convolution equations in bounded domains in spaces with weighted norms, Mat. Sb., 69(1966), 65-110.

[61] S. Zaremba, Sur in probleme mixte relatif a l'équation de Laplace, Bull. de l'Académie des Sciences de Cracovie, Classe des Sciences Mathématiques et Naturelles, Series A, 1910, pp. 313-344.

Department of Mathematics and Statistics, Georgetown University, Washington D.C. 20057, USA.

Department of Mathematics, Fu Jen Catholic University, Taipei 242, Taiwan, ROC.

E-mail: chang@georgetown.edu

College of Science, Tianjin University of Technology and Education, Tianjin, 300222, P.R. China.

E-mail: lyu@math.uni-potsdam.de

Institute of Mathematics, University of Potsdam, Am Neuen Palais 10, D-14469 Potsdam, Germany.

E-mail: schulze@math.uni-potsdam.de 